PNE-242F NUCLEAR EXPLOSIONS-PEACEFUL APPLICATIONS (TID-4500, 43rd Ed.)

\title{
THE SEDAN EVENT
}

\author{
M. D. Nordyke \\ M. M. Williamson, U.S. Army \\ Corps of Engineers \\ Lawrence Radiation Laboratory \\ University of California \\ Livermore, California
}

April 1965 


\section{DISCLAIMER}

This report was prepared as an account of work sponsored by an agency of the United States Government. Neither the United States Government nor any agency Thereof, nor any of their employees, makes any warranty, express or implied, or assumes any legal liability or responsibility for the accuracy, completeness, or usefulness of any information, apparatus, product, or process disclosed, or represents that its use would not infringe privately owned rights. Reference herein to any specific commercial product, process, or service by trade name, trademark, manufacturer, or otherwise does not necessarily constitute or imply its endorsement, recommendation, or favoring by the United States Government or any agency thereof. The views and opinions of authors expressed herein do not necessarily state or reflect those of the United States Government or any agency thereof. 


\section{DISCLAIMER}

Portions of this document may be illegible in electronic image products. Images are produced from the best available original document. 


\section{CONTENTS}

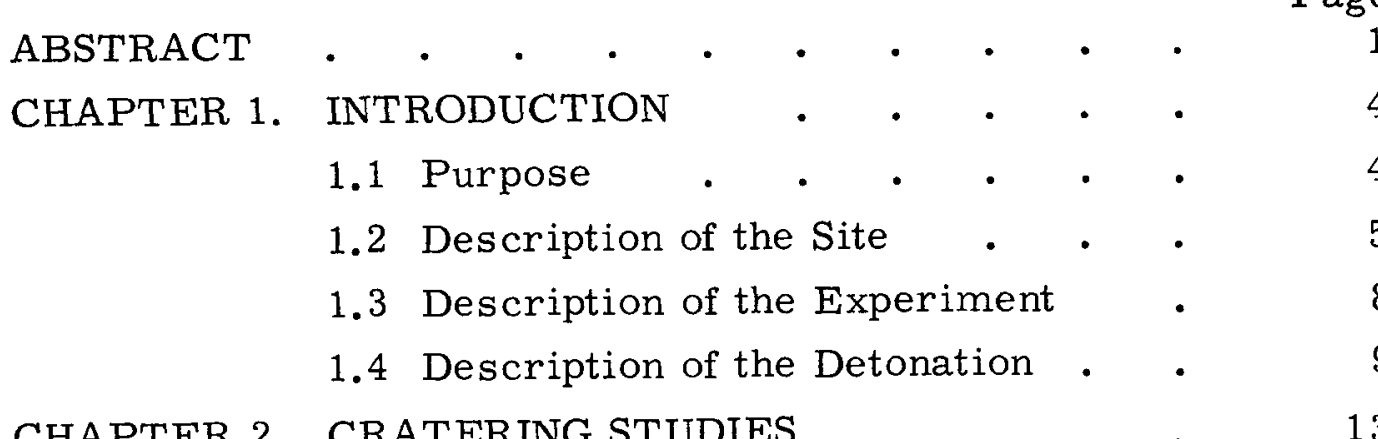

CHAPTER 2 CRATERING STUDIES $\quad \cdot \quad \cdot \quad \cdot \quad \cdot \quad 13$

2.1 Summary of Past Cratering Experience in Desert Alluvium . . 13

2.2 Prediction of Sedan Crater

Dimensions $\quad . \quad$. $\quad . \quad$. $\quad . \quad 13$

$2.2 .1 \mathrm{~W}^{1 / 3.4}$ Scaling . . . $\quad 13$

$2.2 .2 \mathrm{~W}^{1 / 4}$ Scaling . . . 13

2.2.3 Overburden Scaling . • • 16

2.2.4 Russian Data • • • 17

2.3 Crater Results . . . . . . 20

2.4 Discussion and Conclusions - Crater

Data • • . . .

2.4.1 Comparison of Results with
Predictions

2.4.2 Scaling Implications . . 31

2.4.3 Chemical-Nuclear Efficiency 32

2.4.4 Subsidence Crater Data - 33

2.4.5 Nuclear. Cratering Curves
for Alluvium $\quad . \quad . \quad 37$

2.5 Conclusions . $\quad$. . . . $\quad 38$

CHAPTER 3 FALLOUT STUDIES . • • • • • 42

3.1 Summary of Past Experience . . $\quad 42$

3.1 .1 Generál • • • • • . 42

3.1.2 Relationship of Sedan to Past
Nuclear Cratering Events .

3.2 Predictions $\quad$. . . . . . 43 
CONTENTS (Continued)

Page

3.2.1 Activity Production . . 43

3.2.2 Cloud Dimensions . . 44

3.2.3 Fallout Distribution . . 45

3.3 Results . . . . . . 45

3.3.1 Meteorology . . . 45

3.3.2 Cloud Development . • 45

3.3.3 Discussion of Cloud Development • . • • . 48

3.3.4 Radiation Field Measurements 48

3.3.5 Pattern Construction . . 49

3.3.6 Discussion of Pattern 49

3.3.7 Pattern Analysis and
Comparison to Predictions .

3.4 Discussion $\quad$ • $\quad$. $\quad . \quad$. 57

3.4.1 Radioactivities . . . 57

3.4.2 Fallout Distribution . . . 61

3.5 Conclusions . • • . . . 62

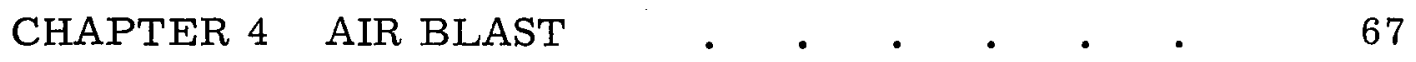

4.1 Close-In Air Blast $\quad$. $\quad . \quad$. $\quad 67$

4.1.1 Experimental Results $\quad$. $\quad 67$

4.1.2 Previous Experience . . $\quad 67$

4.1.3 Comparison of Sedan Overpressures with Previous Experience • . . . 69

4.1.4 Air Blast Impulse . . 70

4.1.5 Discussion and Conclusions $\quad 70$

4.2 Long Range Air-Blast . . . 74

4.2.1 Experimental Plan • . 74

$\begin{array}{lll}\text { 4.2.2 } & \text { Long Range Air-Blast } \\ \text { Transmission Factors } & \text {. } & 74\end{array}$ 
CONTENTS (Continued)

Page

4.2.3 Comparison of Sedan Results with Previous Experience.

4.2.4 Extrapolation of Sedan Results to Larger Yields • • • • $\quad 76$

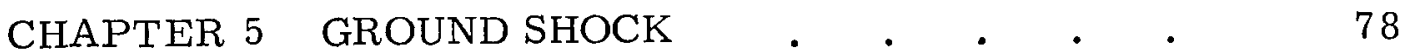

5.1 Experimental Plan . . . . 78

5.2 Experimental Results . . . 78

5.3 Discussion and Conclusions . . 81

CHAPTER 6 MASS DISTRIBUTION $\quad$ • . • • $\quad 84$

6.1 Ejecta and Throwout Thickness . 84

6.1.1 Experimental Plan and

Results . . . . $\quad 84$

6.1.2 Discussion and Conclusions 87

6.2 Impact Craters $\quad . \quad 0 \quad . \quad 0 \quad 88$

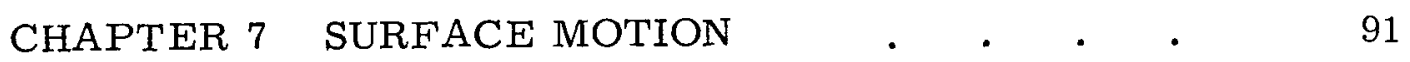

7.1 Experimental Plan . . . . 91

7.2 Experimental Results . . . 91

7.3 Discussion . . . . . . . 93

7.4 Conclusion . $\quad$. $\quad$. $\quad$. 97

7.5 Acknowledgment $\quad$. $\quad$. $\quad 98$ 


\section{ABSTRACT}

Project Sedan was a 100-kt nuclear cratering experiment conducted in 1962 as a part of the Plowshare Program. To document the many phenomena related to nuclear excavation that occur in such a detonation, a large number of technical programs were conducted on the Sedan event. The purpose of Sedan was to (1) extend knowledge of cratering effects to the 100-kt range of yields, and (2) provide data on the general nature of the safety problems to be encountered by nuclear cratering detonations.

The crater resulting from the detonation had an apparent crater radius of 185 meters (608 feet) and a depth of 98.5 meters (323 feet). The volume of the apparent crater was about $5.1 \times 10^{6}$ $\mathrm{m}^{3}\left(6.6 \times 10^{6} \mathrm{yd}^{3}\right)$, corresponding to the removal of about 7.6 $\times 10^{9} \mathrm{~kg}\left(8.4 \times 10^{6}\right.$ tons $)$. The lip ranged in height from 5.5 to 29 meters above the preshot elevation and had a volume of about 3.2 $\times 10^{6} \mathrm{~m}^{3}\left(4.2 \times 10^{6} \mathrm{yd}^{3}\right)$. Comparison of these dimensions with past experience leads to the conclusion that nuclear cratering explosions in the region of optimum depth of burst result in craters with radii about 10-20 percent smaller than equivalentyield chemical explosives and with depths about the same.

Sedan produced a dust cloud 50 percent larger than predicted. This larger cloud was due mainly to the neutral atmosphere that existed to an altitude of $2700 \mathrm{~m}$ and the large volume of gas resulting from the high moisture content (3-6 times greater than assumed) of the alluvium. The cloud path and fallout pattern were strongly affected by terrain effects on the wind.

Nearly five times more radioactivity than predicted came down in the local fallout pattern, showing a need for re-examination of the methods used to extrapolate the relationships between radioactivity, depth of burst, and yield to the: Sedan event. Highest exposure dose to any off-site resident was 100 to $275 \mathrm{mR}$. 
Ex post facto calculations show that fallout prediction techniques are reasonably good when the input data are accurate.

Results from close-in air blast measurements indicated that Sedan produced peak overpressures at ranges of 300 to 5000 meters that were four to five times larger than would have been expected from previous experience with chemical explosives. These overpressures give rise to close-in air-blast transmission factors of 0.08 to 0.2 over the range of measurement as compared to predicted values of 0.015 to 0.05 . Predicted and actual close-in air-blast impulses and impulse transmission factors, however, agreed well. It is hypothesized that the higher overpressures are attributable to the presence of much higher cavity pressures in the nuclear cavity and the earlier scaled venting time for a largeyield shot such as Sedan. Long-range air-blast signals gave transmission factors of 0.2 , in excellent agreement with chemical explosive experience at this scaled depth of burial.

Ground shock from Sedan fell well below the prediction functions developed by USC and GS for smaller-yield nuclear explosions in alluvium. But comparison with data from a similaryield, contained explosion indicates that there may be no consistent reduction in ground motion due to the shallow burial depth of Sedan. Earth motions from Sedan were influenced by the presence of the Yucca fault. Motions on the same side of the fault as Sedan were about a factor of two greater than those on the opposite side.

Mass distribution and ejecta studies on Sedan show that the total volume of ejecta, including the lip, represents about 58 percent of the apparent crater volume. Fifty percent of the ejecta mass was within 2 crater radii of ground zero, 80 percent was within 3 crater radii and 97 percent within 10 crater radii ( 2000 meters). The distribution of ejecta allowed construction of a functional relationship for mass, range, and yield that predicts 
the distribution for the 0.5-kt Scooter explosion within a factor of 1.5 to 2.0. A large number of secondary craters were produced in the area around the Sedan crater by impact of agglomerated masses of material. Impact craters with diameters as large as 10 meters were noted out to ranges of 1300 meters and smaller ones were recorded out to as far as 2140 meters.

Observed surface motion in the Sedan event agrees very well with the model of gas acceleration developed at LRL and previous experience with initial spall velocities. The length of the period of free fall between the initial spall motion and the beginning of the observable gas acceleration phase does not scale from chemical to nuclear explosions in proportion to the cube root of the yield, but will require the development of a more detailed and sophisticated model. 


\section{CHAPTER 1 \\ INTRODUCTION}

Project Sedan was a 100-kt nuclear cratering experiment conducted at the Nevada Test Site in 1962. This event was sponsored by the Atomic Energy Commission's Plowshare Program for the industrial and civil application of nuclear explosives and was conducted under the technical direction of the Lawrence Radiation Laboratory, Livermore, California. To document the many phenomena that occur in such a detonation, many technical programs were conducted on the Sedan event. These programs were related primarily to the development of an understanding of nuclear cratering and the safety problems involved in its use for nuclear excavation projects. This report is intended to present a general summary of the results from those experiments of primary importance to nuclear excavation technology.

\subsection{PURPOSE}

In general terms, the purpose of the Sedan event was to (1) extend knowledge of cratering effects and phenomenology to the 100 -kt range of yields and (2) provide data on the general nature of the safety problems to be encountered by nuclear cratering detonations. Most nuclear excavation projects such as harbors and canals will require the use of nuclear explosives in the range of $100 \mathrm{kt}$ to $10 \mathrm{Mt}$. Previous cratering experience with both nuclear explosives and high explosives (H. E.) had been limited to about $1 \mathrm{kt}$ or below. ${ }^{1}$ Empirical scaling laws had been developed from these data for predicting cratering results from explosions with equivalent charge weights between $100 \mathrm{~kg}$ and $1 \mathrm{kt}$. ${ }^{*}$ However,

\footnotetext{
*An "equivalent charge weight" of $1 \mathrm{kt}\left(10^{6} \mathrm{~kg}\right)$ is defined as an explosive unit releasing an energy of $10^{12}$ calories. TNT releases approximately 1000 calories per gram or about $10^{12}$ calories per million kilograms. 
serious questions had been raised regarding the use of these laws for predicting the results from high-yield ( $>100 \mathrm{kt}$ ) explosions. Concern had been expressed that megaton craters would be significantly smaller than the empirical scaling law for low yields would permit.

The yield of the Sedan event was chosen on the basis that it was significantly larger (about 100 times) than any previous buried cratering explosion and was close enough to the megaton range of yields so that any effects of major importance at these yields would be detectable. Thus Sedan was designed to answer these questions about cratering effects as well as to provide data on the nature of the safety problems related to radioactivity, ground shock, and air blast. This report is a summary of the cratering results from Sedan and the current status of our knowledge of the cratering effects of nuclear explosives in general.

\subsection{DESCRIPTION OF THE SITE}

The site chosen for Sedan (see Figure 1.1) was at the north end of Yucca Valley at the Nevada Test Site in Area 10 near several other nuclear craters (Jangle U and Teapot ESS), ${ }^{2}$ and a large number of high explosive craters (Scooter, Stagecoach). ${ }^{3,4}$ The medium in this general area is alluvial in nature, consisting of fragments of tertiary volcanic (rhyolite and tuff), limestone, quartzite, conglomerate, and shale that have washed down into the alluvial valley. Considerable variations in size, composition and caliche cementation have been observed throughout the general area, with interlacing thin layers of material of different textures. It has generally been described as a sand-gravel mixture.

A major north-south fault occurs approximately 1300 meters west of the Sedan ground zero point. The thickness of alluvium in Area 10 is somewhat variable, ranging from a depth of about 430 meters at the Sedan ground zero and tapering out to zero at 


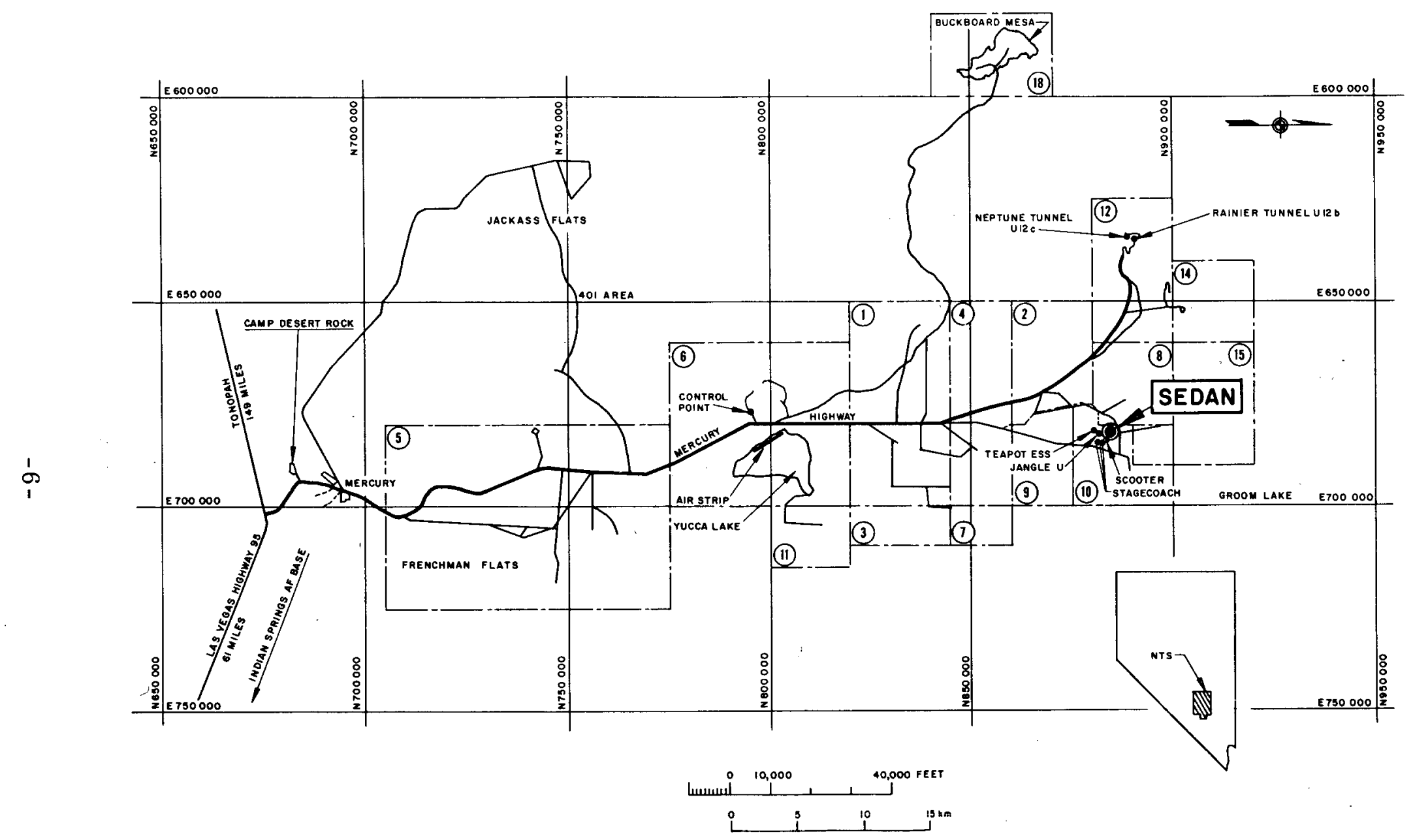

Figure 1.1 Map showing Sedan site. 
the hills east of Sedan. The thickness of the alluvium west of the Yucca Fault is estimated at about 300 meters. ${ }^{5}$ Underlying the alluvium in the Sedan area is over 300 meters of weakly cemented volcanic tuff that has physical properties very similar to those of the alluvium found at depth. The density of the alluvium has been measured by many investigators and has been found to be quite variable, being generally in the range between 1.5 and 1.7 with the higher number being more common at depths exceeding 30 meters. Chemical composition of the alluvium in Area 10 is shown in Table 1.1, where chemical analyses are given for three composite shallow surface samples, two from Teapot ESS area, and one from the Sedan area. No attempt was made to retain in situ water content for these samples. To better define moisture content as well as $\mathrm{CO}_{2}$ content, two additional samples were sealed in the field and subsequently analyzed only for water and $\mathrm{CO}_{2}$. The results of these tests are also given in Table 1.1 and show the water and $\mathrm{CO}_{2}$ content to be extremely variable. These results, as well as results for other areas of Yucca Flat, have led to the general conclusion that water content of the alluvium varies from about 10 percent in the first 30 meters to about 20 percent at a depth of 300 meters. Over 1,000,000 gallons of water were lost during the drilling of the Sedan emplacement hole and thereby added to the environment. Based on data from Thardarson et al. ${ }^{6}$ it is estimated that the water table in the Sedan area is at a depth of about 600 meters.

Elastic properties of the alluvium are extremely difficult to measure and vary widely from sample to sample. Compressional wave velocities can be obtained from Scooter free-field measurements as being $1100 \pm 100 \mathrm{~m} / \mathrm{sec}$. Bulk compressibilities for two samples representing fine and coarse grain sizes ranged from 18.7 to 3.4 kbars respectively. ${ }^{7}$ 
TABLE 1.1 CHEMICAL COMPOSITION OF ALLUVIUM, AREA 1.0, NTS ${ }^{a}$

\begin{tabular}{|c|c|c|c|c|c|}
\hline \multirow[b]{2}{*}{ Elemental Oxide } & \multicolumn{5}{|c|}{ Composite Sample Number - Per centage by Weight } \\
\hline & Teapot ESS & Teapot ESS & Sedan & Sedan & Sedan \\
\hline Silica dioxide & $68.8 \%$ & $69.1 \%$ & $74.5 \%$ & & \\
\hline Alluvium dioxide & 11.1 & 10.9 & 8.0 & & \\
\hline Calcium oxide & 4.8 & 5.0 & 5.3 & & \\
\hline Magnesium oxide & 1.0 & 0.93 & 1.8 & & \\
\hline Ferric oxide & 3.1 & 3.0 & 4.6 & & \\
\hline Sodium oxide & 1.7 & 1.7 & 0.27 & & \\
\hline Potassium oxide & 2.7 & 2.7 & 0.11 & & \\
\hline Phosphorus pentoxide & 0.02 & 0.01 & 0.38 & & \\
\hline Titanium dioxide & 0.30 & 0.26 & 0.22 & & \\
\hline Manganous oxide & 0.07 & 0.06 & 0.05 & & \\
\hline Water $\left(\mathrm{T}<105^{\circ} \mathrm{C}\right)$ & $1.10^{b}$ & $1.06^{b}$ & $0.73^{b}$ & 9.2 & 2.6 \\
\hline $\begin{array}{l}\text { Water }\left(105^{\circ} \mathrm{C}<\mathrm{T}\right. \\
\left.<1800^{\circ} \mathrm{C}\right)\end{array}$ & $2.09^{\mathrm{C}}$ & $2.10^{\mathrm{C}}$ & $2.78^{\mathrm{c}}$ & 12.3 & 6.1 \\
\hline $\begin{array}{l}\text { Carbon dioxide }(\mathrm{T} \\
\left.<105^{\circ} \mathrm{C}\right)\end{array}$ & & & 2.3 & 5.0 & 1.0 \\
\hline $\begin{array}{l}\text { Carbon dioxide } \\
\left(105^{\circ} \mathrm{C}<\mathrm{T}<1800^{\circ} \mathrm{C}\right)\end{array}$ & 2.9 & 3.0 & 2.7 & 6.5 & 1.1 \\
\hline
\end{tabular}

${ }^{\mathrm{a}}$ Abbot A. Hanks, Inc., private communications April, 1961 and February, 1964.

$\mathrm{b}_{\text {Weight loss assumed to be water. These samples do not }}$ represent the in situ water content of alluvium.

${ }^{c}$ Weight loss minus $\mathrm{CO}_{2}$ - assumed to be water.

\subsection{DESCRIPTION OF THE EXPERIMENT}

A nominal 100-kt thermonuclear device, in which less than 30 percent of the energy came from fission, was used for the Sedan event. Neutron-absorbing material was placed around the device to reduce the quantities of radioactivity induced in the soil. The device was emplaced in a 36-inch-diameter cased hole at a 
depth of 194 meters (635 feet). The region around the device was filled with more neutron absorber (colemanite) and the rest of the hole filled to the surface with dry sand. Coordinates of ground zero were ${ }^{8}$

\section{Geodetic}

$\mathrm{N} 37^{\circ} 10^{\prime} 37^{\prime \prime}$

$\mathrm{W} 116^{\circ} 02^{\prime} \quad 43^{\prime \prime}$
Nevada Control Zone Grid

N 884,000 feet

E 681,000 feet

While the primary purpose of Sedan was to study cratering effects, a large number of technical programs were included to study all phases of the detonation. These programs included fallout collection and measurement, and bio-environmental effects studies. Studies for documenting ground shock and air blast, both on-site and off-site, were also performed. Programs for studying close-in ground motion by means of high-speed photography and underground pressure transducers were included as well as a program for documenting the total mass distribution. Complete details of these programs and their results are given in the documents listed at the end of this report.

\subsection{DESCRIPTION OF THE DETONATION}

Sedan was detonated at 1000:00.147 PDT, (1700:00.147 MT) on 6 July $1962 .{ }^{9}$ A roughly hemisphericál dome 180-250 meters in diameter rose to a height of about 90 meters in 3 seconds at which time venting of large quantities of incandescent gases occured. The material in the dome continued to rise to a height of about 600 meters. Figure 1.2 is a sequence of photographs of this action. As this volume of earth fell back to the ground, it generated a base surge that expanded radially to a distance of approximately $4.0 \mathrm{~km}$ cross-wind and $3.2 \mathrm{~km}$ upwind. The main cloud resulting from the venting gases rose to a height of about 3650 meters above the desert, where it was topped by inversion conditions in the atmosphere. 


\section{VARIOUS STAGES OF CRATERING EXPLOSIONS \\ THROUGH DEVELOPMENT OF BASE SURGE \\ 100 KT AT 193 METERS IN ALLUVIUM (SEDAN)}

SCALE FOR ALL PICTURES; BAR $=300$ METERS
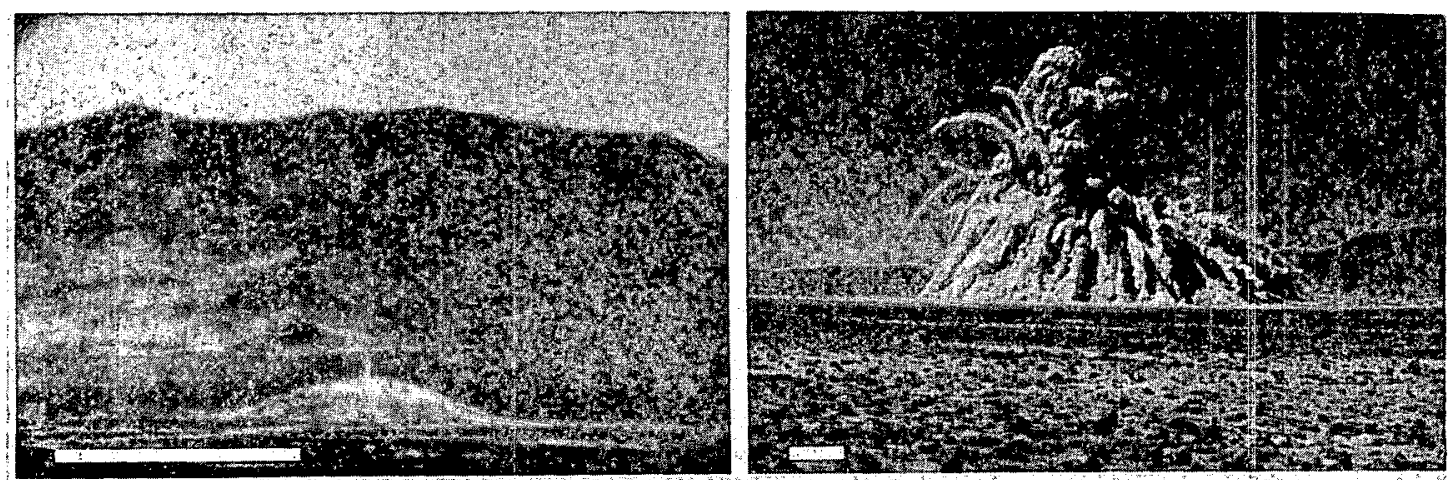

1. TIME $=\mathrm{H}+\mathrm{t} 1.9 \mathrm{sec}$.

4. TIME $=\mathrm{H}+27 \mathrm{sec}$
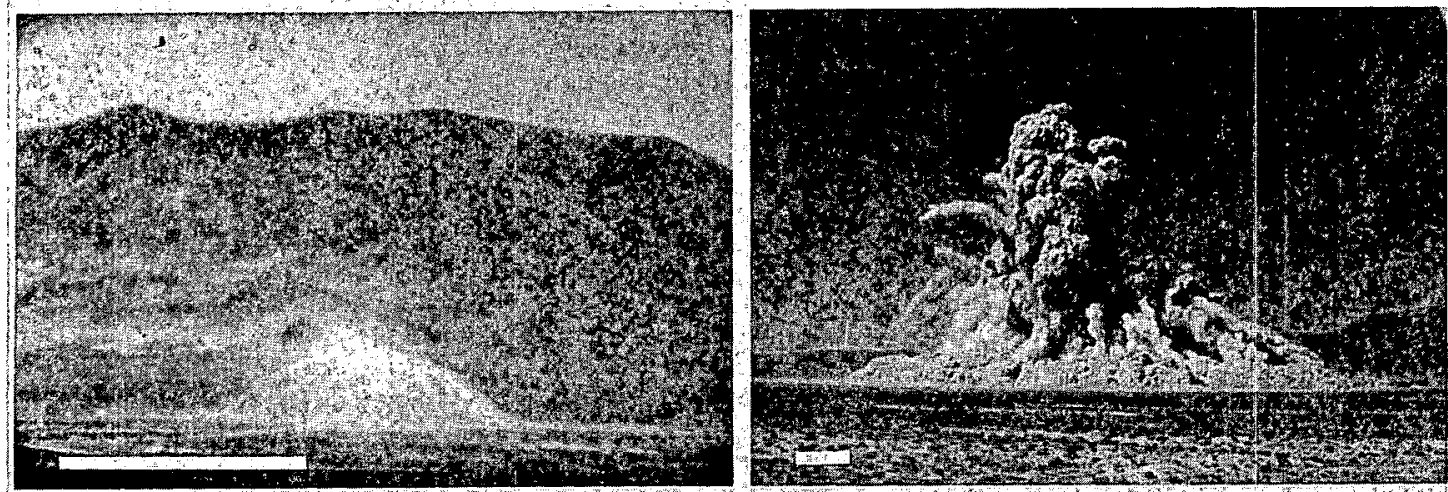

2. TIME $=\mathrm{H}+2.8 \mathrm{sec}$

5. TIME $=\mathrm{H}+39 \mathrm{sec}$
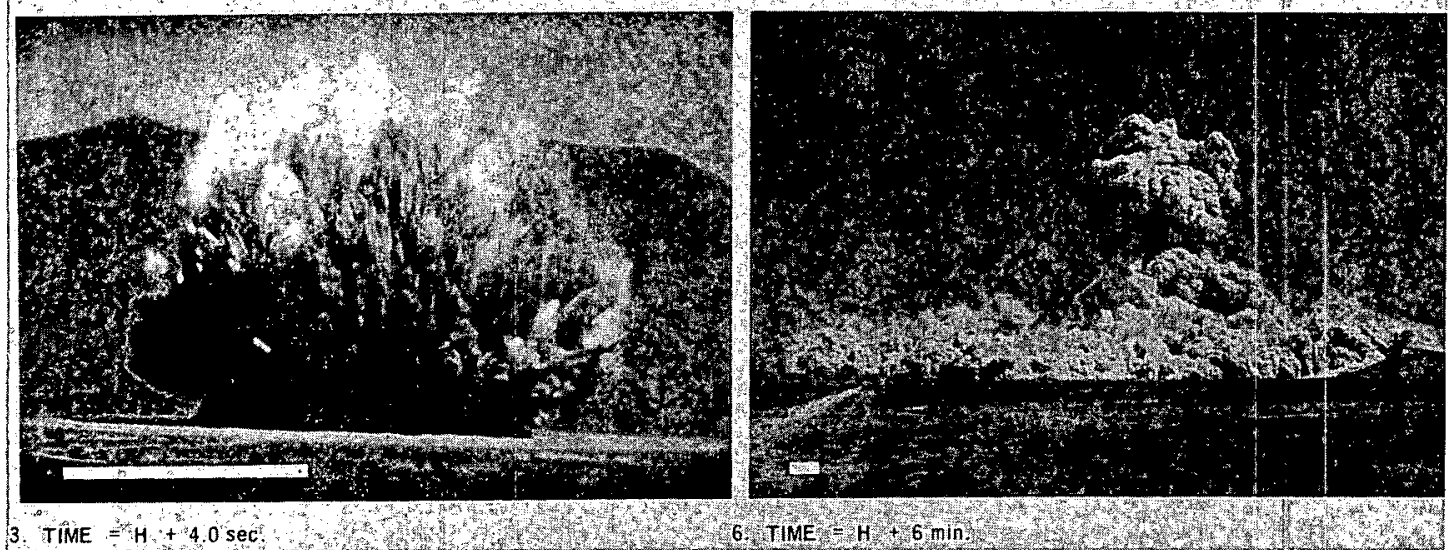

Figure 1.2 Sedan time sequence. 
Radiochemical analysis of cloud samples gave a yield for the Sedan explosive of $100 \pm 15 \mathrm{kt}$. Comparison of hydrodynamic and shock time-of-arrival measurements with theoretical calculations for an underground nuclear explosion on a hydro-plasticelastic computer code gave a yield of $110 \pm 20 \mathrm{kt}$. Because of its greater reliability and smaller uncertainty, the radiochemistry yield of $100 \pm 15 \mathrm{kt}$ has been adopted as the official yield of the Sedan event and has been used in all subsequent analyses of Sedan data. 


\section{REFERENCES}

1. M. D. Nordyke, "An Analysis of Cratering Data from Desert Alluvium, " Journal of Geophysical Research, 1965-1974, 1962.

2. M. D. Nordyke, "Nuclear Craters and Preliminary Theory of the Mechanics of Explosive Crater Formation, "Journal of Geophysical Research, 3439-3459, 1961.

3. W. R. Perret, and others, "Project Scooter, Final Report," Sandia Corporation, SC-4602(RR), 1963.

4. L. J. Vortman, and others, "Project Stagecoach, Final Report, "Sandia Corporation, SC-4596(RR), 1962.

5. W. P. Williams and T. H. Eale, U. S. Geological Survey, Personal Communication, 1962.

6. W. Thardarson, and others, "Ground Water Test Well D, Nevada Test Site, Nye County, Nevada," U.S. Geological Survey Report, TEI-803, 1962.

7. J. T. Cherry and D. Stephens, Lawrence Radiation Laboratory, private communication.

8. W. V. Mickey, "Seismic Effects from a High-Yield Nuclear Cratering Experiment in Desert Alluvium, Project Sedan, Final Report, "U.S. Coast and Geodetic Survey, PNE-213F, 1963.

9. E. M. Mathews and B. F. Murphy, "Timing and Firing, Project Sedan, Final Report, " Edgerton, Germeshausen and Grier, Inc., PNE-233F, 1963. 


\section{CHAPTER 2}

\section{CRATERING STUDIES}

\subsection{SUMMARY OF PAST CRATERING EXPERIENCE IN DESERT ALLUVIUM}

A summary of cratering experience with both chemical and nuclear explosions in desert alluvium prior to Sedan is shown in Figures 2.1 and 2.2. Also shown is a curve fit to the $116-\mathrm{kg}$ (2561b) data by an inverted data regression analysis. ${ }^{1}$ It has been shown that cratering data obtained with charge weights $W$ ranging from $18,200 \mathrm{~kg}(40,000 \mathrm{lbs})$ to $455,000 \mathrm{~kg}(1,000,000 \mathrm{lb})$ can best be correlated with the above curve by scaling the crater dimensions in proportion to $\mathrm{w}^{1 / 3.4}$. This procedure has been used to scale all of the cratering data in desert alluvium to $1 \mathrm{kt}$ for Figures 2.1 and 2.2 .

\subsection{PREDICTION OF SEDAN CRATER DIMENSIONS}

2.2.1 $\mathrm{W}^{1 / 3.4}$ Scaling: The crater dimensions expected for the Sedan crater were calculated from the data in Figures 2.1 and 2.2 by using $\mathrm{W}^{1 / 3.4}$ scaling to scale from 1 to $100 \mathrm{kt}$. This gave the predicted dimensions shown in Table 2.1 in the column "W $1 / 3.4$ scaling." The uncertainty of $\pm 5 \%$ shown was based on the standard deviations resulting from the above regression analysis. These predictions are also shown in Figures 2.1 and 2.2.

Since $\mathrm{w}^{1 / 3.4}$ scaling had been empirically determined only for yields of less than $1 \mathrm{kt}$, there was no assurance it would be operative at the 100-kt level of yields. Further, several other methods of predicting the Sedan crater size were available that predicted significantly different results.

2.2.2 $\mathrm{W}^{1 / 4}$ Scaling: Scaling of the cratering data in proportion to the fourth root of the explosive yield has been suggested by a number of authors as a result of consideration of the potential 


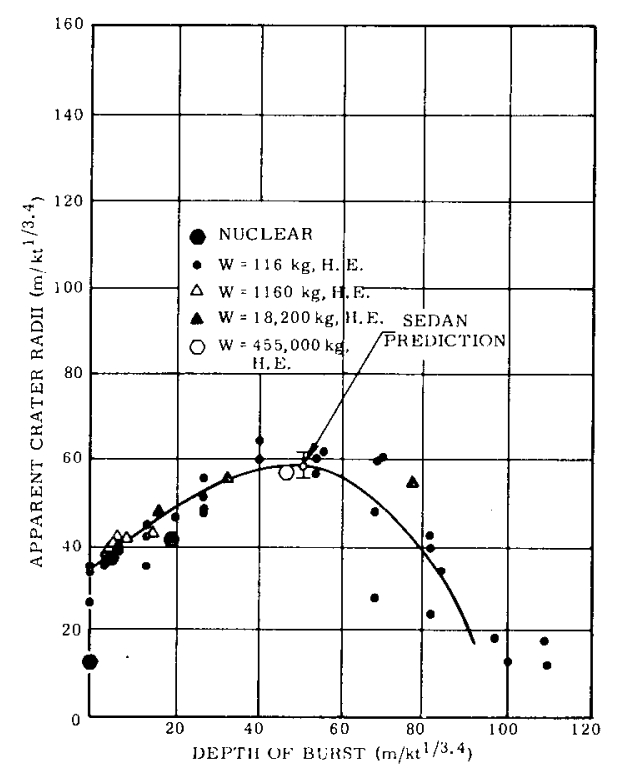

Figure 2.1 Apparent crater radius vs depth of burst; Nevada desert alluvium, high explosives and nuclear explosives, $\mathrm{W}^{1 / 3.4}$ scaling.

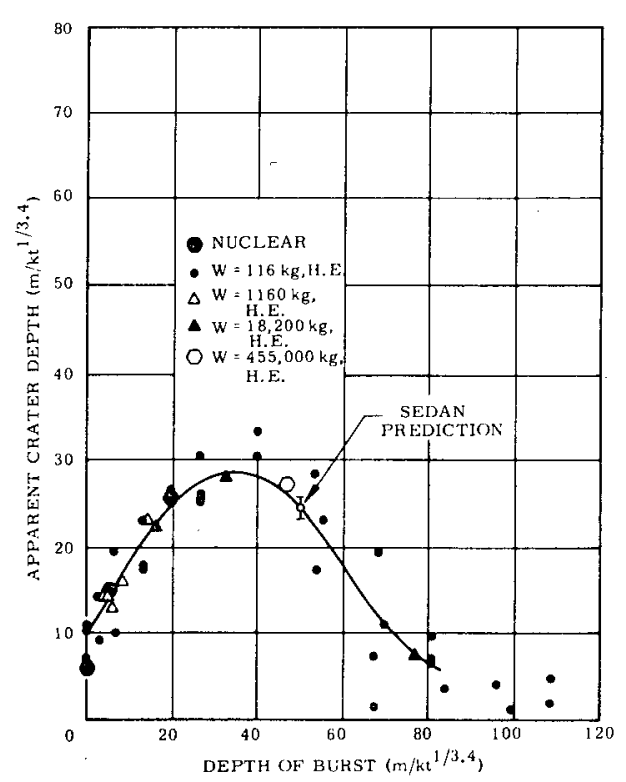

Figure 2.2 Apparent crater depth vs depth of burst; Nevada desert alluvium, high explosives and nuclear explosives, $\mathrm{W}^{1 / 3.4}$ scaling. 
energy of the mass ejected from the crater. If we let the mass of the ejected material be $M$, we have

$$
\mathrm{M}=\mathrm{k}_{1} \mathrm{R}^{2} \mathrm{D} \rho
$$

The potential energy of this mass after the explosion is increased in proportion to its elevation by a height that is proportional to the crater depth, D. Thus we have for the potential energy increase

$$
\Delta \text { P.E. }=k_{2} \text { M D g }=k_{1} k_{2} R^{2} D^{2} g .
$$

If we assume a parabolic-shaped crater and no subsurface compaction, the average elevation increment is at least $(1 / 5) \mathrm{D}$. Assuming $\mathrm{W}^{1 / 3}$ scaling and $\mathrm{R} \cong 50 \mathrm{w}^{1 / 3}, \mathrm{D} \cong 30 \mathrm{w}^{1 / 3}$, and we have

$$
\begin{aligned}
\Delta \mathrm{P} . \mathrm{E} . & =\frac{\pi \mathrm{R}^{2} \mathrm{D} \rho}{2} \times \frac{\mathrm{Dg}}{5} \\
& =\frac{\pi \rho \mathrm{g}}{10}(50)^{2}(30)^{2} \mathrm{~W}^{4 / 3} \\
& =7.0 \times 10^{5} \rho \mathrm{g} \mathrm{W}^{4 / 3} .
\end{aligned}
$$

If we let $\rho=1.5 \mathrm{~g} / \mathrm{cm}^{3}=1500 \mathrm{~kg} / \mathrm{m}^{3}$, we have for the amount of the explosion energy devoted to potential energy

$$
\begin{aligned}
\Delta \mathrm{P} . \mathrm{E} . & \cong 10^{10} \times \mathrm{W}^{4 / 3} \text { joules } \\
& =0.0024 \mathrm{~W}^{4 / 3} \mathrm{kt} \text { equivalent. }
\end{aligned}
$$

The fraction $\mathrm{W}$ of the total energy going into potential energy is then $\Delta$ P.E. $/ \ddot{W}=0.0024 \dot{W}^{1 / 3}$, where $\mathrm{W}=$ explosion yield in kilotons of equivalent energy. For $W=100 \mathrm{kt}, \Delta \mathrm{P} . \mathrm{E} . / \mathrm{W}=1.1 \%$.

Thus, this simple argument shows that as the scale of the explosion increases toward a megaton or larger yields, $\mathrm{w}^{1 / 3}$ scaling implies that a larger and larger proportion of the energy is going into potential energy. If the fraction of the energy going 
into potential energy is to remain a constant proportion of the total explosive energy, we can see that

$$
\frac{\Delta P . E .}{W}=\text { constant }=\frac{k_{1} k_{2} R^{2} D^{2} g}{W}
$$

or

$$
\mathrm{R}^{2} \mathrm{D}^{2}=\mathrm{k}_{3} \mathrm{~W}
$$

which can be satisfied dimensionally by assuming

$$
(\mathrm{R}, \mathrm{D}) \propto \mathrm{W}^{1 / 4}
$$

It seems most probable that the actual behavior of a cratering explosion should lie between these two alternatives (i. e., the empirical $\mathrm{W}^{1 / 3.4}$ scaling and $\mathrm{W}^{1 / 4}$ scaling) but that $\mathrm{W}^{1 / 4}$ scaling would certainly appear to be a limiting behavior at large yields. Predictions using $\mathrm{W}^{1 / 4}$ scaling to extrapolate the kiloton data in Figures 2.1 and 2.2 to $100 \mathrm{kt}$ are given in Table 2.1.

\section{TABLE 2.1 PREDICTED CRATER DIMENSIONS, PROJECT SEDAN}

\begin{tabular}{lrllc}
\hline & \multicolumn{2}{c}{ Apparent Crater Radius } & \multicolumn{2}{c}{ Apparent Crater Depth } \\
\hline & meters & feet & meters & feet \\
$\mathrm{W}^{1 / 3.4}$ Scaling & $228 \pm 11$ & $748 \pm 34$ & $97 \pm 5$ & $319 \pm 16$ \\
$\mathrm{~W}^{1 / 4}$ Scaling & $180 \pm 18$ & $590 \pm 60$ & $54 \pm 5$ & $177 \pm 18$ \\
Overburden Scaling & $145 \pm 30$ & $475 \pm 100$ & $50 \pm 35$ & $170 \pm 120$ \\
Russian Data & $252-298$ & $827-980$ & $103-134$ & $338-440$ \\
\hline
\end{tabular}

\subsubsection{Overburden Scaling: Another method of presenting} the cratering data shown in Figures 2.1 and 2.2 had been proposed by A. Chabai. This method is alternatively termed "gravity scaling," ${ }^{2}$ or "overburden scaling." 3 This approach, based on dimensional analysis, scales crater dimensions and depth of burst in proportion to the quantity

$$
\eta=\left[\frac{W^{\prime}}{\rho g Z+p}\right]^{1 / 3}
$$


where

$\rho=$ average density of cratering medium between detonation point and the ground surface, $\mathrm{kg} / \mathrm{m}^{3}$

$\mathrm{g}=$ acceleration of gravity, $\mathrm{m} / \mathrm{sec}^{2}$

$Z=$ depth of burst, meters

$\mathrm{p}=$ atmospheric pressure at ground surface $=0.86 \times 10^{5}$ newtons $/ \mathrm{m}^{2}$ at 4000 feet altitude

$\mathrm{W}^{\prime}=$ energy, joules.

Figures 2.3 and 2.4 present the desert alluvium apparent crater data when scaled by the factor $\eta$. The dashed lines shown in Figures 2.3 and 2.4 are fits to the data from Reference 2 . It should be noted that this approach leads to $\mathrm{W}^{1 / 3}$ scaling for small yields where $\mathrm{p}>>\mathrm{gZ}$ and to $\mathrm{W}^{1 / 4}$ scaling for large yields where $\mathrm{p}<<\rho \mathrm{gZ}$.

If this method of data analysis is used to predict the crater dimensions for the Sedan crater, the values given in Table 2.1 in the column "overburden scaling" result. The uncertainties given are estimated from Figures 2.3 and 2.4 as being consistent with the scatter of the data. These predictions are indicated on Figures 2.3 and 2.4 .

2.2.4 Russian Data: Over the past 15 to 20 years scientists in the USSR have devoted a great amount of theoretical and experimental effort to cratering with large-scale explosions. ${ }^{4}$ Their cratering experience is based on explosions which range from a few grams up to as many as 9200 tons of chemical explosives in a single point charge. Based on this body of experience, the following formula was developed by M. M. Boreskov ${ }^{5}$ for calculating the size charges required for the excavation of rock and gravel:

$$
W=K_{B} Z^{3}\left(0.4+0.6 n^{3}\right)
$$




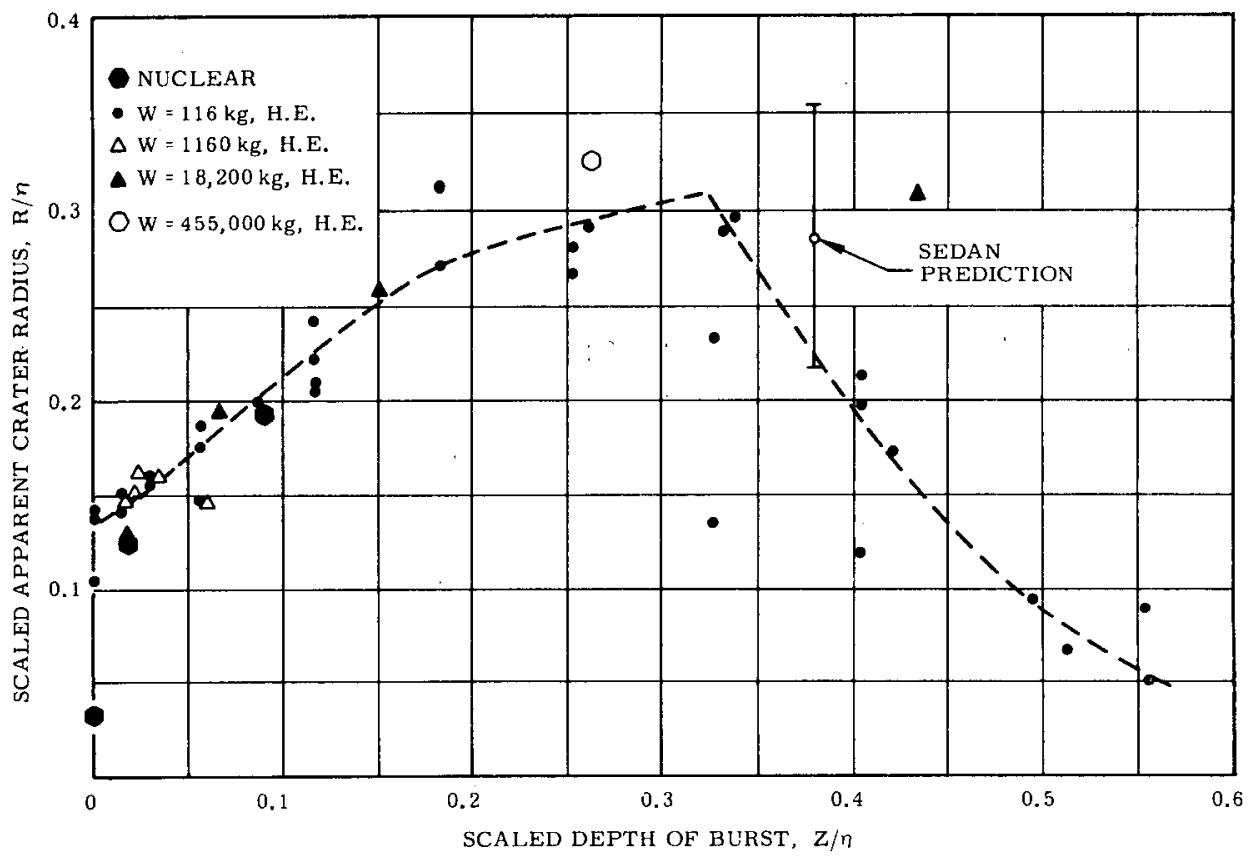

Figure 2.3 Apparent crater radius vs depth of burst.

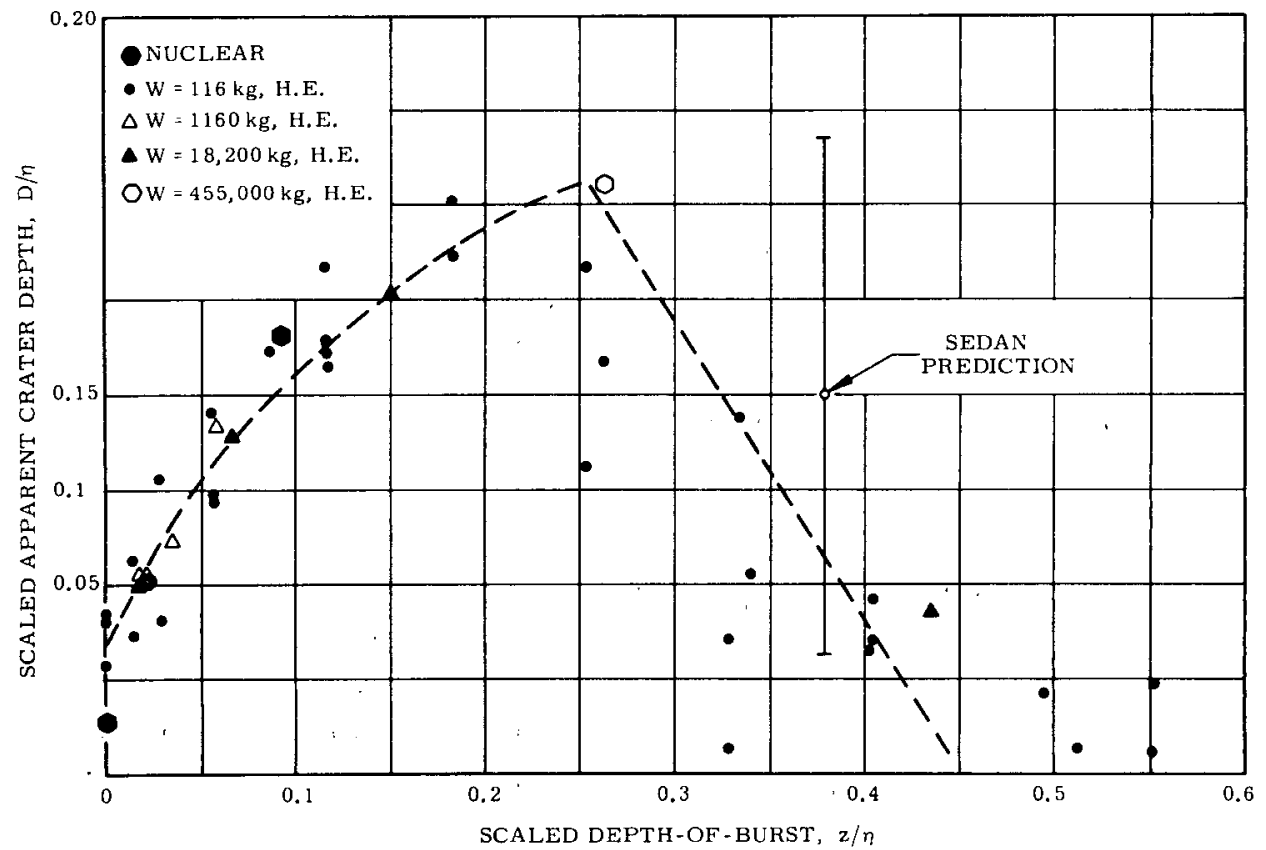

Figure 2.4 Apparent crater depth vs depth of burst. 
where $\mathrm{D}$ is the apparent crater depth in meters. It can be noted that these equations are consistent with similarity laws and the cube-root scaling law.

For cases where $Z>25 \mathrm{~m}$, however, the following formula, based on large-scale crater experience, is recommended ${ }^{6}$ :

$$
\mathrm{W}=\frac{\mathrm{K}_{\mathrm{B}} \mathrm{z}^{3.5}}{5}\left(0.4+0.6 \mathrm{n}^{3}\right) \text {. }
$$

This formula, of course, conforms to $\mathrm{W}^{1 / 3.5}$ scaling procedures. Determination of the value for $\mathrm{K}_{\mathrm{B}}$ applicable to NTS desert alluvium can be made from data in the Handbook of the Soyuzvzryoprom Explosion Trust. ${ }^{6}$ There is indicated that for sand the value for $K_{B}$ should be 1.5-1.7. Use of the value for $K_{B}$ of 1.7 gives a prediction for the Sedan crater radius of 252 meters (827 feet) and 103 meters (338 feet) for the depth.

Theoretical calculation of crater parameters by G. I. Pokrovskii ${ }^{5}$ based on lifting the ejected mass to a height sufficient to permit it to escape from the crater leads to the expression

$$
W=\frac{\rho}{28000} z^{3.5}\left(1+n^{2}\right)^{2} .
$$

This equation, with a density of $\rho=1500 \mathrm{~kg} / \mathrm{m}^{3}$ and a depth of burst of 194 meters, predicts a radius of 345 meters and a depth of 165 meters.

Pokrovskii further recommends the following equation for explosions greater than $0.3 \mathrm{kt}$ :

$$
\mathrm{W}=\frac{\rho^{2} z^{4}}{6.6 \times 10^{8}}\left(1+n^{2}\right)^{5 / 2}
$$

This equation predicts a Sedan crater radius of 298 meters (980 feet) and a depth of 134 meters (440 feet).

All of the foregoing predictions are summarized in Table 2.1 . 
where

$\mathrm{W}=$ charge weight of chemical explosive, $\mathrm{kg}$;

$\mathrm{Z}=$ depth of burst, meters;

$\mathrm{n}=$ index of explosions effort $=$ ratio of apparent crater radius $R$ to depth of burst $Z$; and

$\mathrm{K}_{\mathrm{B}}$ is a constant (ranging from 1.0 to 2.0 ) that depends on the type of rock or ground being considered.

Apparent crater depths are given by the equation

$$
\mathrm{D}=\frac{2 \mathrm{n}-1}{3} \cdot \mathrm{Z} \text {, }
$$

\subsection{CRATER RESULTS}

Figure 2.5 is an aerial photo showing the Sedan crater and the general crater area. Figure 2.6 is a photo taken from one side of the crater showing the true crater outcrop around the rim and the nature of the talus slopes of fallback material. Figures 2.7 and 2.8 show contour maps of the Sedan area before and after the explosion. Figure 2.9 shows an outline of the apparent crater as a solid line and the crest of the lip as a dashed line. Figure 2.10 shows four cross sections through the Sedan crater together with a circumferential lip profile showing the variations in the lip height. The eight apparent crater radii obtained from these four cross sections are listed in Table 2.2. The average apparent crater radius from these eight radii is $185.3 \pm 2.4$ meters $(608$ \pm 8 feet). The average apparent crater radius $\bar{R}_{A}$ was also calculated from the plan area of the apparent crater to be 186.7 meters (612 feet). For the purpose of analysis, a crater radius for Sedan of 186 meters (610 feet) has been used. The apparent crater depth, measured from the original ground surface along the vertical axis of the crater, is 98.2 meters (322 feet). The deepest point in the crater occurs at a point 15 meters WSW of the center where the crater depth is 98.5 meters (323 feet). 


\section{-}

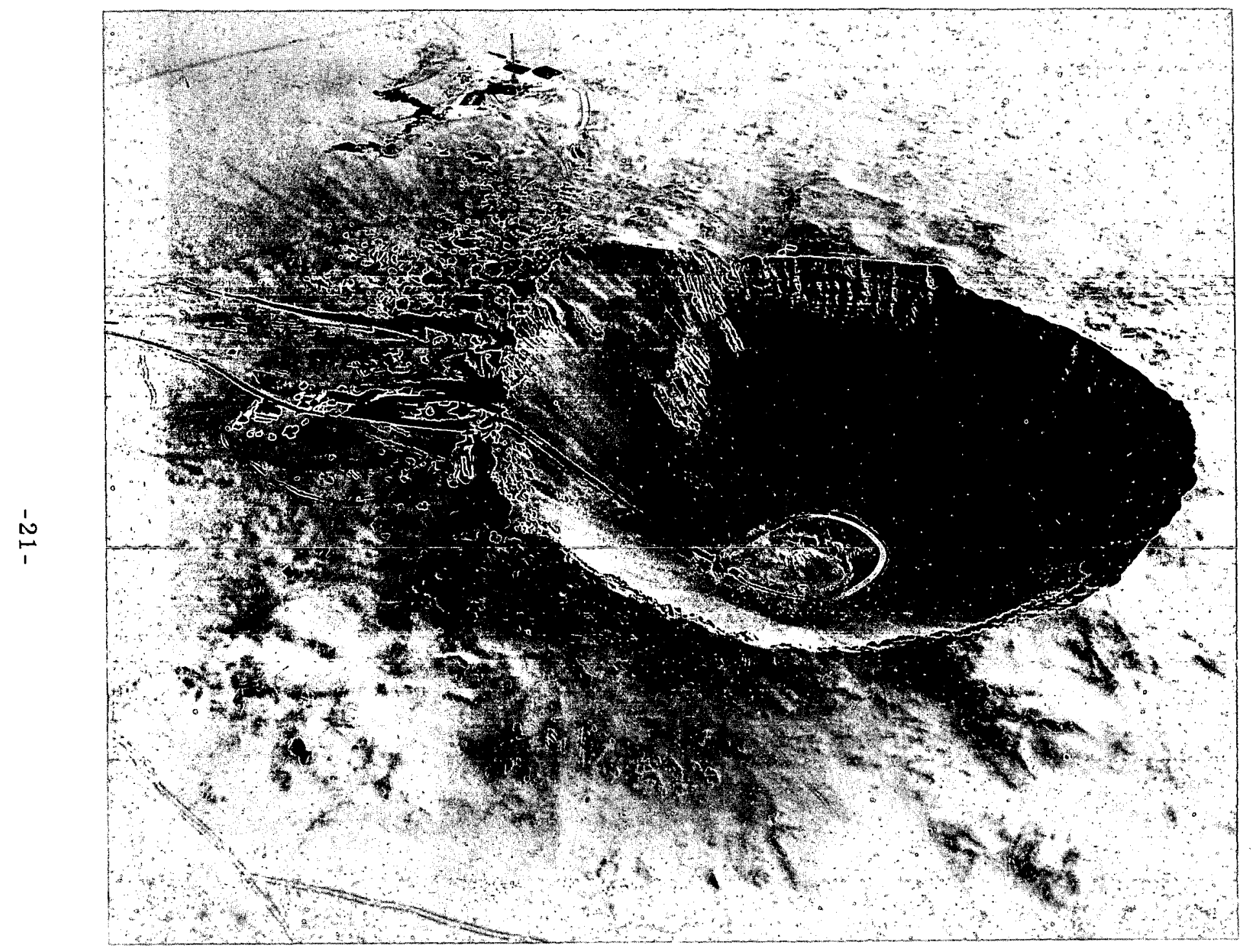

Figure 2.5 Sedan crater. 


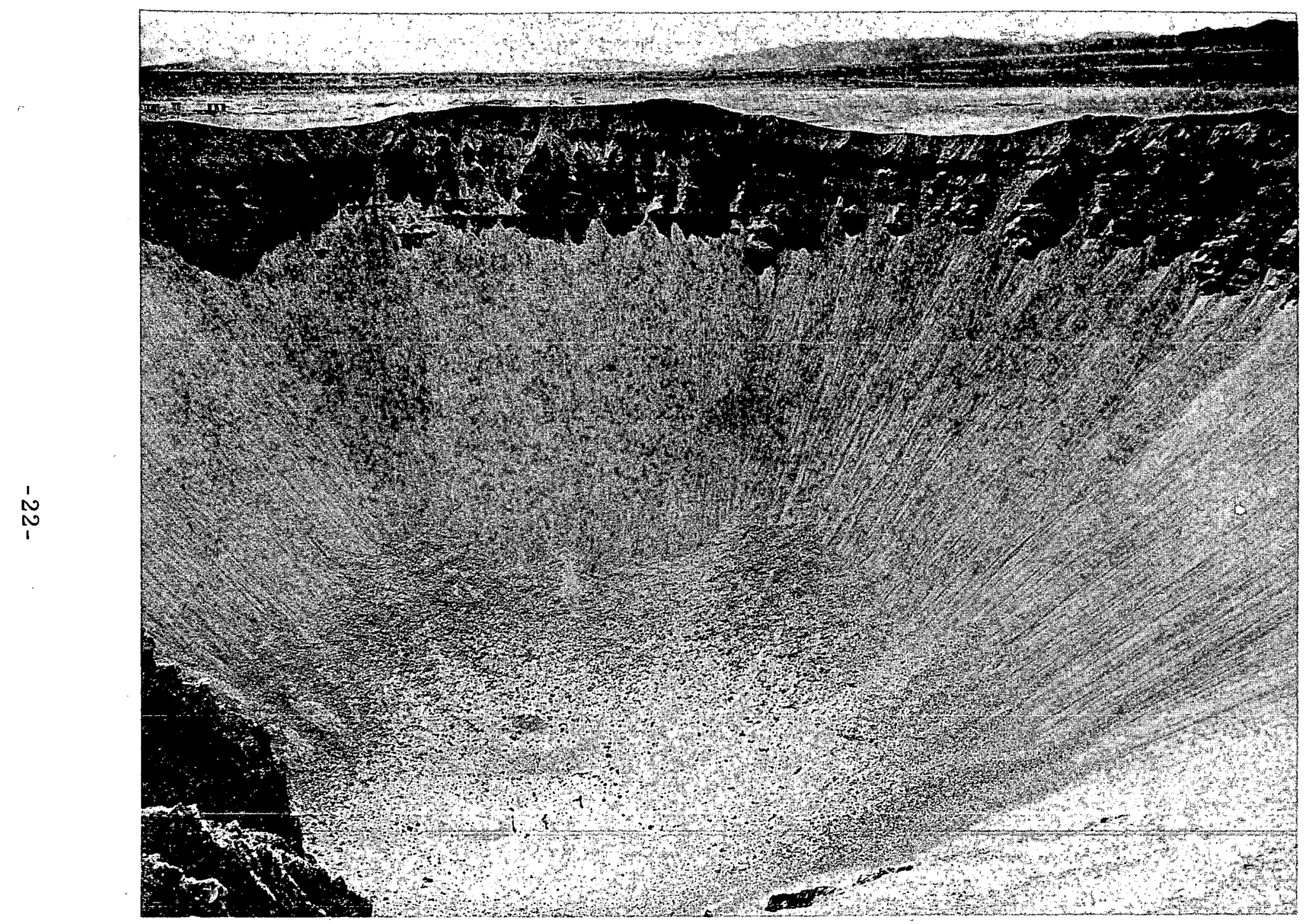

Figure 2.6 Sedan crater seen from lip. 


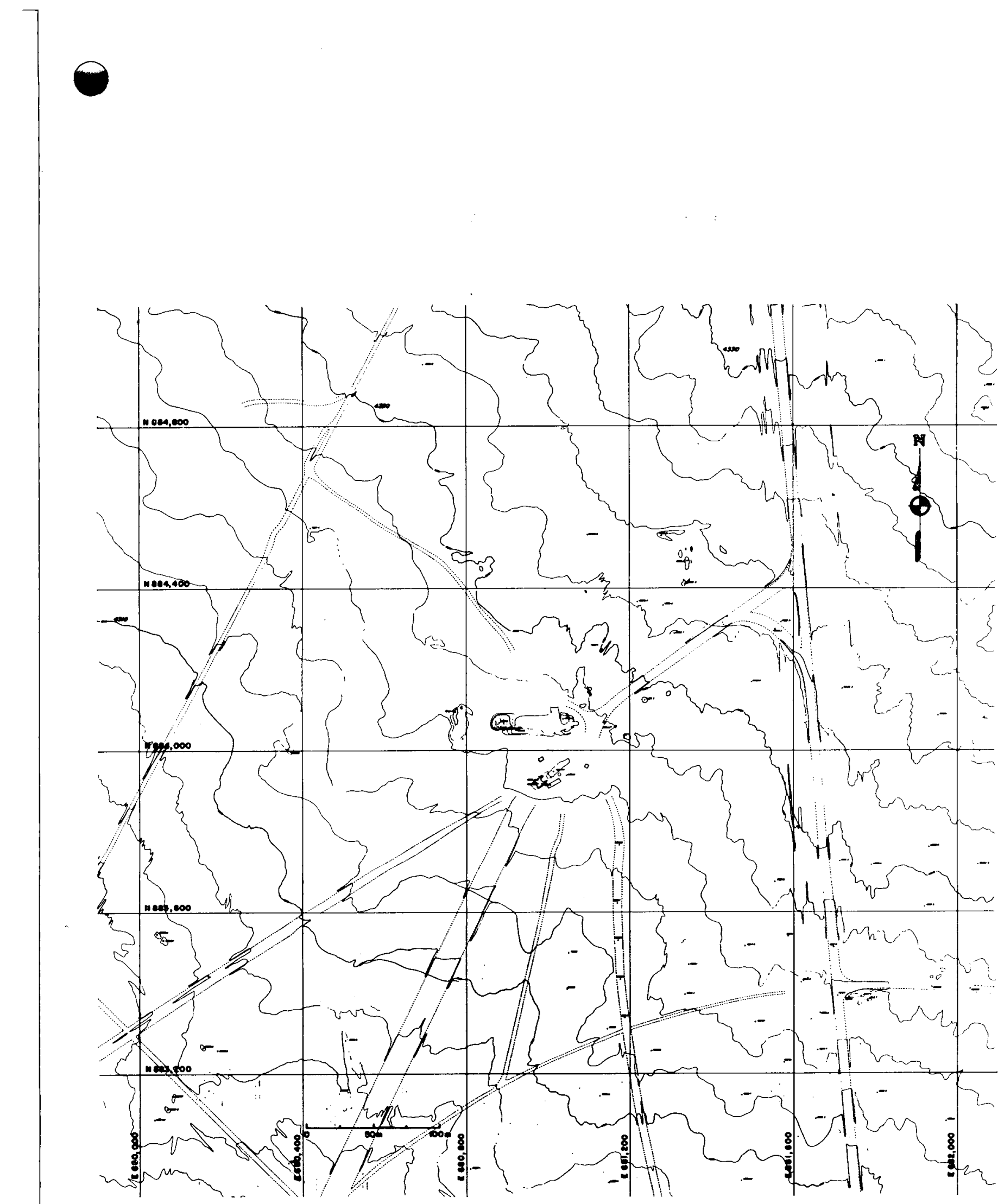




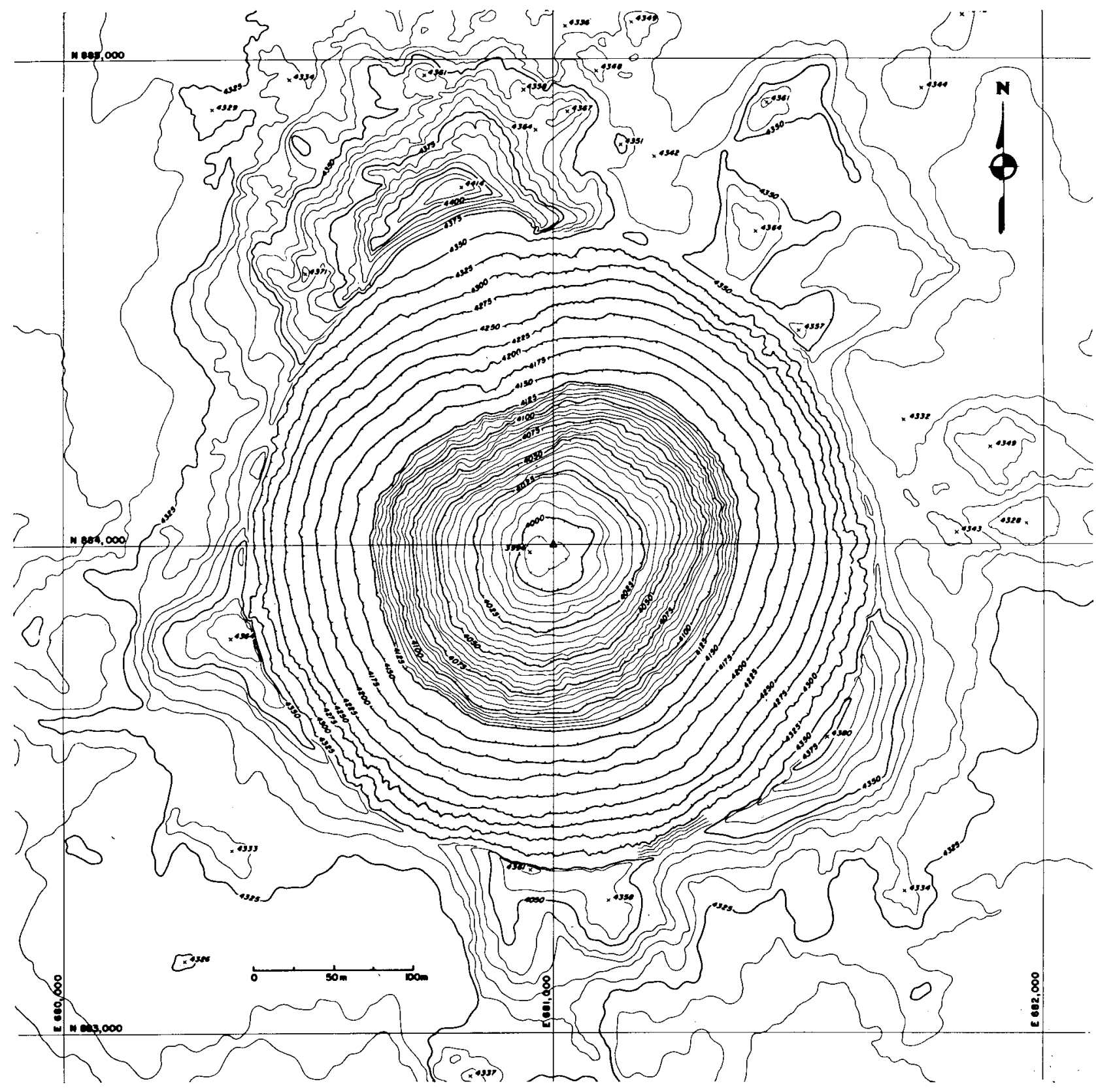

Figure 2.8 Contour map of Sedan area after explosion. 


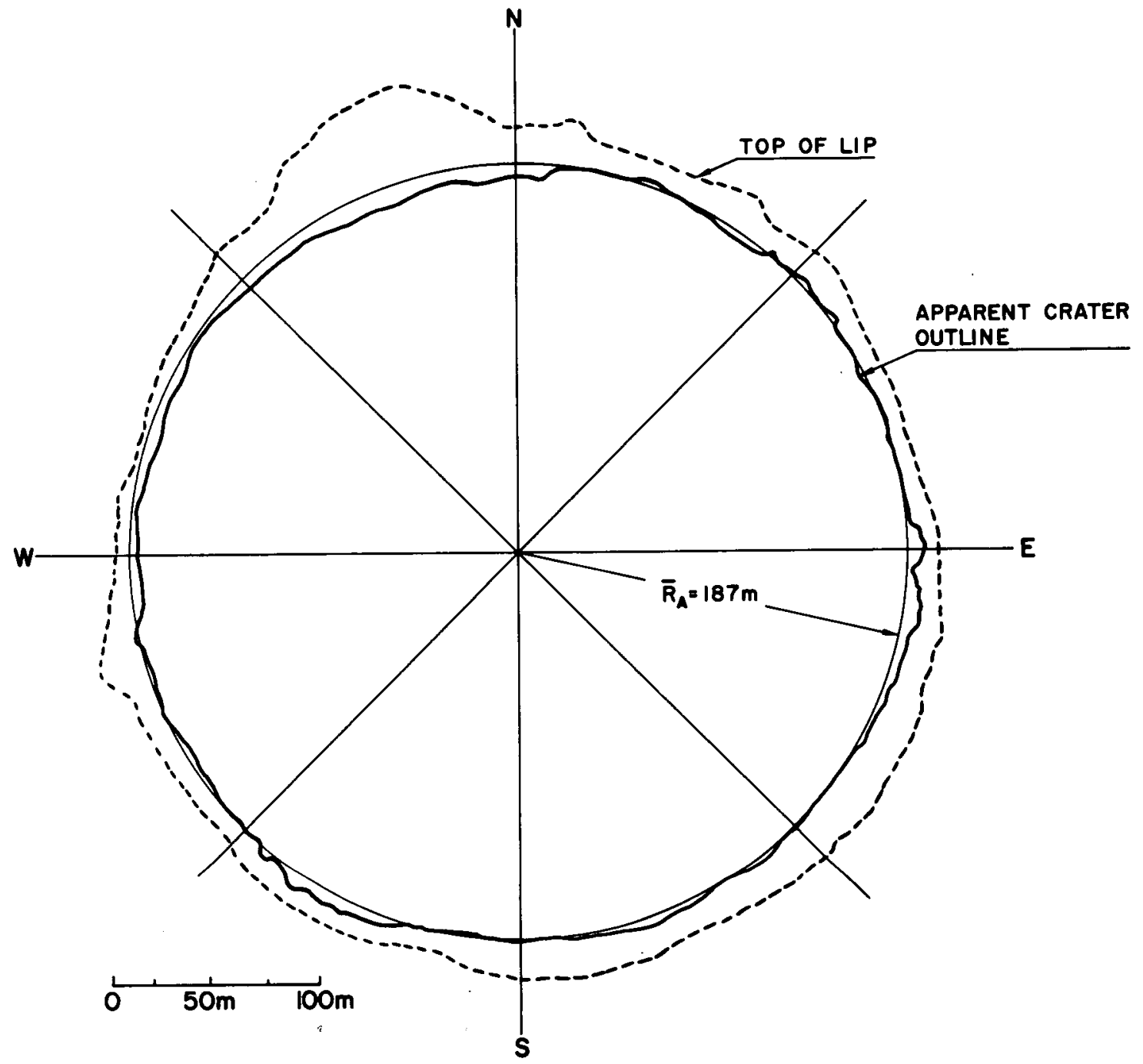

Figure 2.9 Outline of apparent crater and crest of lip. 

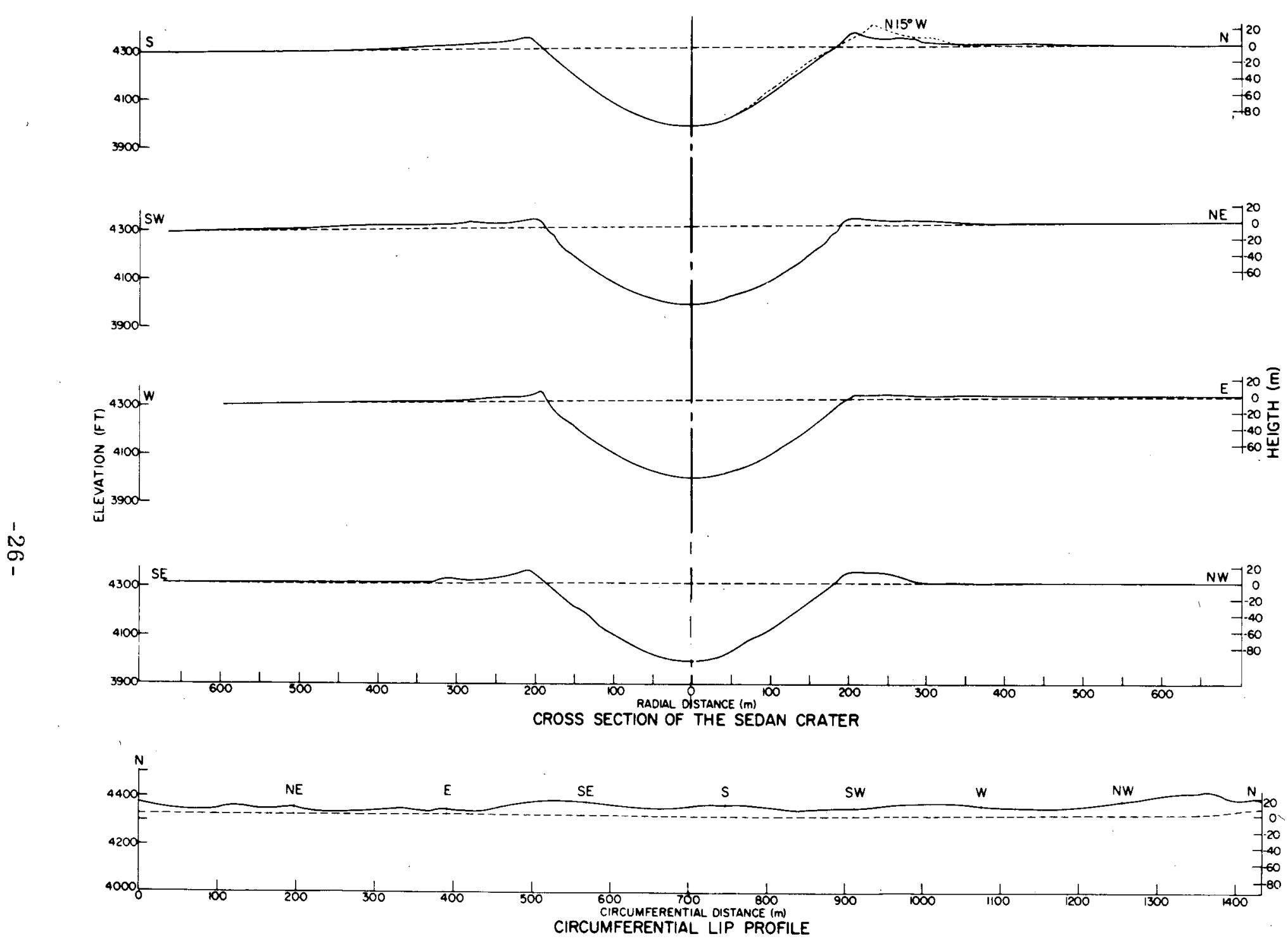

Figure 2.10 Sedan cross sections and circumferential lip profile. 
TABLE 2.2 SEDAN APPARENT CRATER RADII

\begin{tabular}{lc}
\hline Cross -Section Azimuth & Radius \\
\hline & (meters) \\
GZ - North & 182 \\
GZ - South & 190 \\
GZ - Northeast & 185 \\
GZ - Southwest & 185 \\
GZ - East & 190 \\
GZ - West & 183 \\
GZ - Northwest & 182 \\
GZ - Southeast & 185
\end{tabular}

Average Apparent Crater Radii $185.3 \pm 2.4$ meters (608 \pm 8 feet)

As can be seen in Figures 2.9 and 2.10, the shape of the crater is almost exactly circular in plan and parabolic in cross section.

The volume of the crater has been calculated by measuring the area of contours on Figures 2.7 and 2.8 to be $5.07 \times 10^{6} \mathrm{~m}^{3}$ $\left(6.6 \times 10^{6} \mathrm{yd}^{3}\right)^{7}$. This can be compared with a figure of $5.30 \times 10^{6}$ $\mathrm{m}^{3}\left(6.9 \times 10^{6} \mathrm{yd}^{3}\right)$ based on a paraboloid with the above average dimensions. Using an average density for desert alluvium of 1.5 $\mathrm{g} / \mathrm{cm}^{3}$ this corresponds to an apparent crater mass of $7.65 \times 10^{9}$ $\mathrm{kg}$ or $8.42 \times 10^{6}$ tons.

The lip varies in elevation above the original grade from 5.5 to 29 meters. The average lip height, obtained from the projected area of the lip profile, is 13 meters. Carlson ${ }^{7}$ has also calculated the total lip volume to be about $3.2 \times 10^{6} \mathrm{~m}^{3}$ or 4.81 $\times 10^{9} \mathrm{~kg}$.

Of particular interest is the large subsidence area visible on the azimuth of about $\mathrm{N} 15^{\circ} \mathrm{W}$ in Figure 2.8 and in the top profile of Figure 2.10. It is also very apparent on the left side of the crater 
in Figure 2.5. All evidence indicates that this anomaly resulted from an asymmetric initial motion of the ground along that azimuth. The material beyond about 70 meters had an initial motion significantly less than at other azimuths and the material inside 70 meters significantly more, resulting in a vertical slip fault with a strike approximately perpendicular to the radius. Figure 2.11 is a photograph taken at about 1.9 seconds, it clearly shows this anomaly on the left side of the expanding dome. Geologic evidence indicates that a wedge-shaped piece of the lip along this azimuth about 100 meters wide came back down at a much later stage of the explosion than the material on either side. Thus this piece was jammed into the reduced hole left by the portion of the lip on either side which had fallen back earlier, resulting in downward deformation of the sides of the hole and a greatly increased height for the lip at this point. From this picture, it would appear that the same mechanism applied along this azimuth as in the rest of the crater, but for some as yet unexplained geologic reason, was delayed in time for that portion beyond 70 meters. It should be noted that there is no appreciable amount of ejecta or fallback on top of this prominence. ${ }^{8}$

Because of its great height and unusual support geometry, a subsidence failure of this portion of the lip occurred very early, before any visible observation of the crater was possible, resulting in a concave slide mass half-way down on the $\mathrm{N} 15^{\circ} \mathrm{W}$ cross section of the crater (Figure 2.10).

With the exception of the massive subsidence failure the structure was very similar to that noted in earlier craters in alluvium. ${ }^{9,10}$ The lip consisted of uplifted strata topped by overturned material of varying thickness. Figure 2.12 shows a cross section through the Sedan lip constructed by Richards ${ }^{8}$ showing the postshot location of a number of marker beds and the original ground surface and the approximate location of the missing hinge 


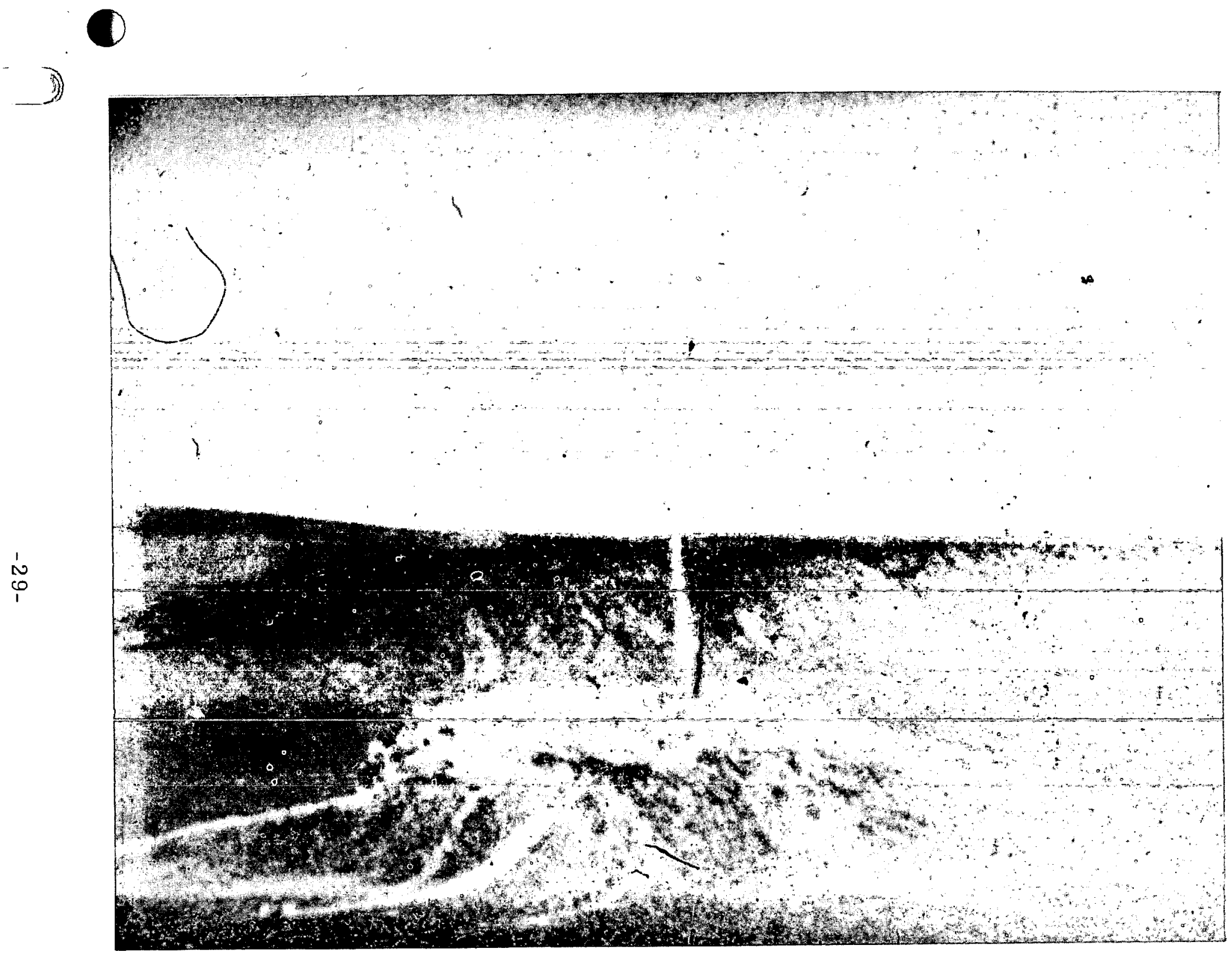

Figure 2.11 Sedan dome at 1.9 seconds. 


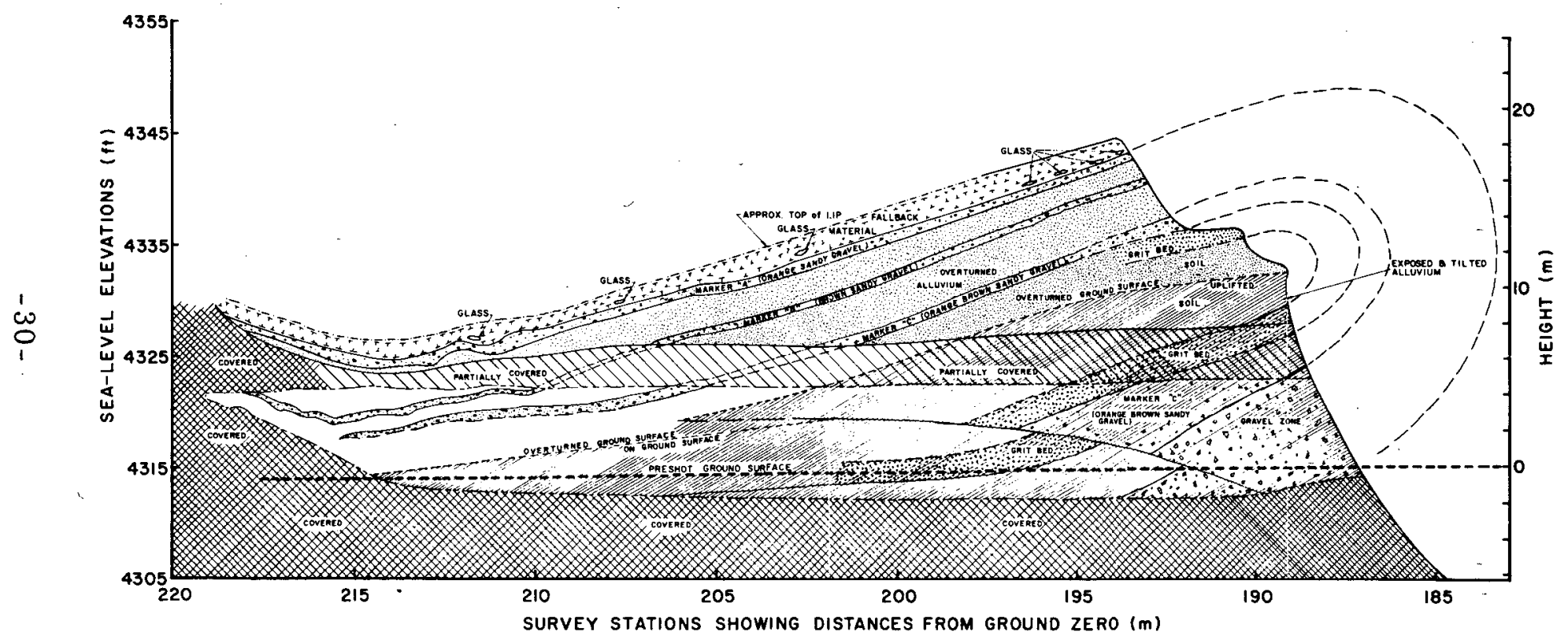

Figure 2.12 Geologic section through the Sedan lip as exposed in cut located on a bearing $\mathrm{N} 58^{\circ} \mathrm{W}$ from G. Z. 
about which all of the overturned material rotated. Further discussion of the geological character of the Sedan crater can be found in PNE- $240^{8}$ and PNE-234. ${ }^{11}$

\subsection{DISCUSSION AND CONCLUSIONS - CRATER DATA}

2.4.1 Comparison of Results with Predictions: The apparent crater radius of 186 meters falls well below that predicted on the basis of $\mathrm{w}^{1 / 3.4}$ scaling from the chemical explosive data as well as those based on the Russian data (see Table 2.1). It is somewhat higher than the upper limit of the overburden scaling prediction but is within the limits of $\mathrm{w}^{1 / 4}$ scaling from the 1 -kt chemical explosive curve. The apparent crater depth of 98 meters is essentially in agreement with the $\mathrm{w}^{1 / 3.4}$ scaling prediction but is almost 100 percent larger than the predictions of $\mathrm{W}^{1 / 4}$ scaling or overburden scaling. The Sedan depth agreed fairly well with the prediction based on the latest Russian data but was significantly lower than would be predicted by the theoretical equations of Pokrovskii.

2.4.2 Scaling Implications: If scaling exponents are calculated ${ }^{1}$ relative to the 1 - kt chemical explosive curves in Figures 2.1 and $2.2, \mathrm{~W}^{1 / 3.9}$ scaling for radius and $\mathrm{W}^{1 / 3.4}$ for depth results. Since the chemical explosive cratering curves were actually derived from $116 \mathrm{-kg}$ data, scaling exponents can be derived by scaling the Sedan dimensions to $116 \mathrm{~kg}$. This procedure results in exponents of $1 / 3.5$ and $1 / 3.4$ for radius and depth, respectively.

The above indeterminancy of the scaling exponent for radius demonstrates one of the major difficulties of empirical scaling laws. If the functional relationships relating crater dimensions at one yield to those at another yield is dependent on the yields involved, the empirical scaling laws that can be derived will vary with the range between the two sets of data that are being compared. Only if the empirical scaling law is independent of yield will the 
same scaling law be derived from different sets of data. Previous experience with determining empirical scaling exponents for 18 ton and 454-ton chemical craters by comparison with 116 -kg data indicated no change in the empirical scaling exponent between 18 tons and 454 tons.

2.4.3 Chemical-Nuclear Efficiency: An alternate method of reconciling nuclear explosive data with chemical explosive data is the use of the concept of a chemical-nuclear explosive efficiency factor, $\epsilon$. This is, by definition, the ratio of the chemical explosive equivalent yield to the nuclear explosive equivalent yield required for an equivalence of cratering effects. Using $\mathrm{w}^{1 / 3.4}$ scaling, this results in an $\epsilon$ for Sedan of $\sim 54$ percent for the crater radius and $\sim 102$ percent for crater depth. Comparable numbers for the two other applicable nuclear craters, Jangle $U$ and Teapot ESS are given in Table 2.3 below. ${ }^{1}$

TABLE 2.3 CHEMICAL-NUCLEAR EFFICIENCIES

\begin{tabular}{lcc}
\hline Event & $\epsilon_{\mathrm{r}}(\%)$ & $\epsilon_{\mathrm{d}}(\%)$ \\
\hline Jangle U & 87 & 135 \\
Teapot ESS & 49 & 132 \\
Sedan & 54 & 102 \\
\hline
\end{tabular}

The nuclear data are fairly consistent with each other under this presentation and indicate a chemical-nuclear explosive efficiency for crater radius that decreases with depth of burial and is about 50 percent for optimum burial. The efficiency for crater depth appears to be somewhat greater than 100 percent for all depths of burial. A 50 percent efficiency factor for optimum depth of burial would correspond to a factor of about 18 percent in scaled depth of burst and apparent crater radius or a scaled increment of about $10 \mathrm{~m} / \mathrm{kt}^{1 / 3.4}$. 
The difference between $\epsilon_{\mathrm{r}}$ and $\epsilon_{\mathrm{d}}$ in Table 2.3 is undoubtedly related to the mechanism of crater formation and the difference between nuclear and chemical explosives. Mechanisms dominant in determining crater depth are not necessarily also dominant in determining crater radius. It is therefore not unreasonable that changes in the physical nature of the explosive would produce different effects on apparent crater radius and depth (see Section 4.1.5).

Data from the $0.42-\mathrm{kt}$ nuclear cratering detonation in basalt, Danny Boy, agree remarkably well with the above conclusion. ${ }^{12}$ The apparent crater radius of the Danny Boy crater was 13 percent smaller than the corresponding chemical explosive data would have predicted, whereas the depth was about right. These factors correspond to an efficiency for apparent crater radius of 62 and 100 percent for depth.

\subsubsection{Subsidence Crater Data: In addition to the Sedan} crater, a large number of "subsidence" craters have recently been made in the alluvial valleys at the Nevada Test Site. These have resulted from the AEC's nuclear weapons development program wherein nuclear explosives were detonated at scaled depths of burial much deeper than Sedan. When a large nuclear explosive is detonated at a depth of burial much deeper than optimum, a large underground cavity is formed which ultimately collapses, resulting in a large subsidence crater at the surface of the ground. Figure 2.13 shows a schematic cross section of such a crater as reconstructed from postshot drill-hole information. Figure 2.14 is a photograph of a typical subsidence crater a few seconds after collapse.

The data points for a large number of these subsidence craters whose scaled depths of burial vary from 100 to 200 $\mathrm{m} / \mathrm{kt}^{1 / 3.4}$ are shown in Figures 2.15 and 2.16 along with all of the chemical and nuclear explosive crater data, including Sedan. 

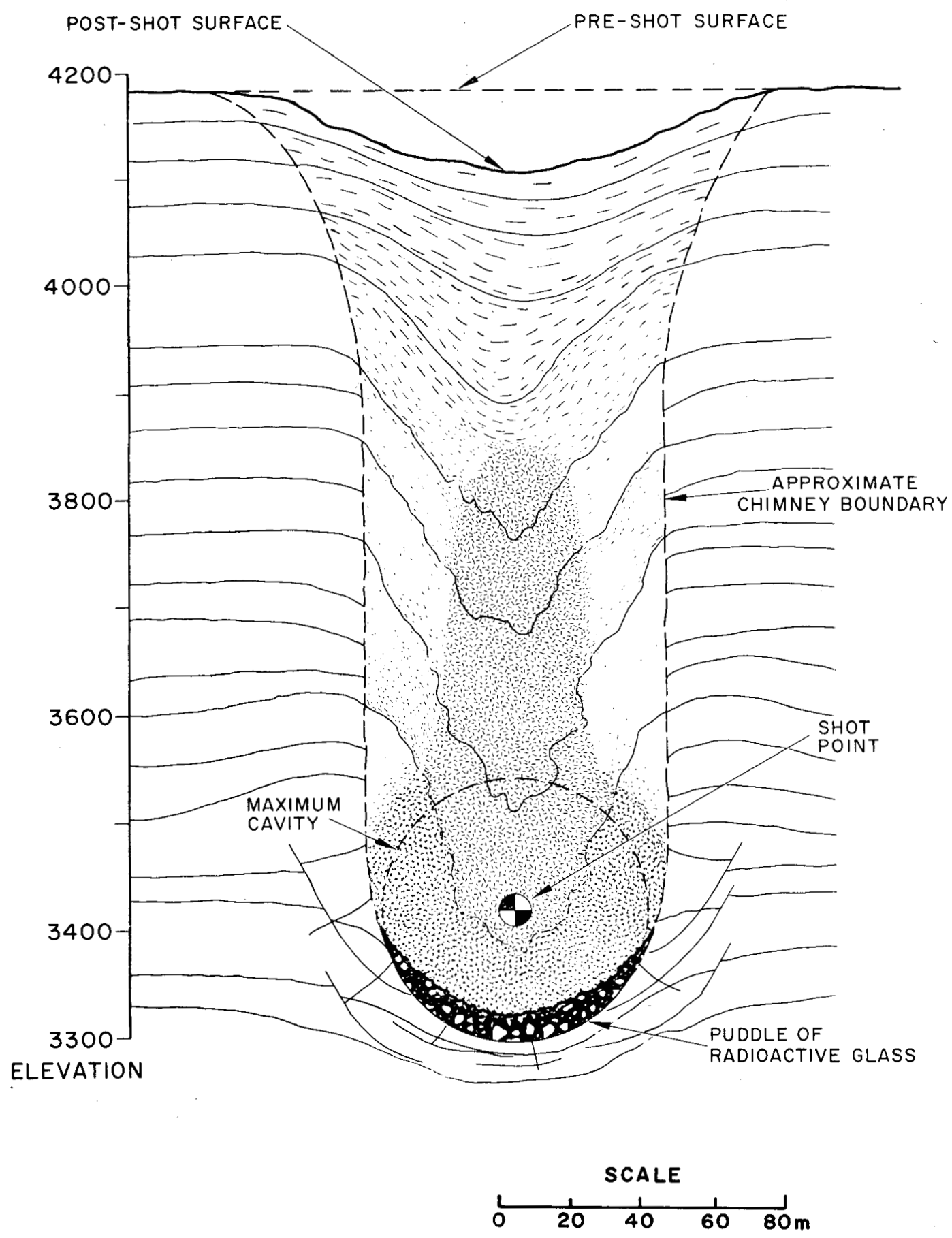

Figure 2.13 Schematic cross section of subsidence crater. 


\section{1}

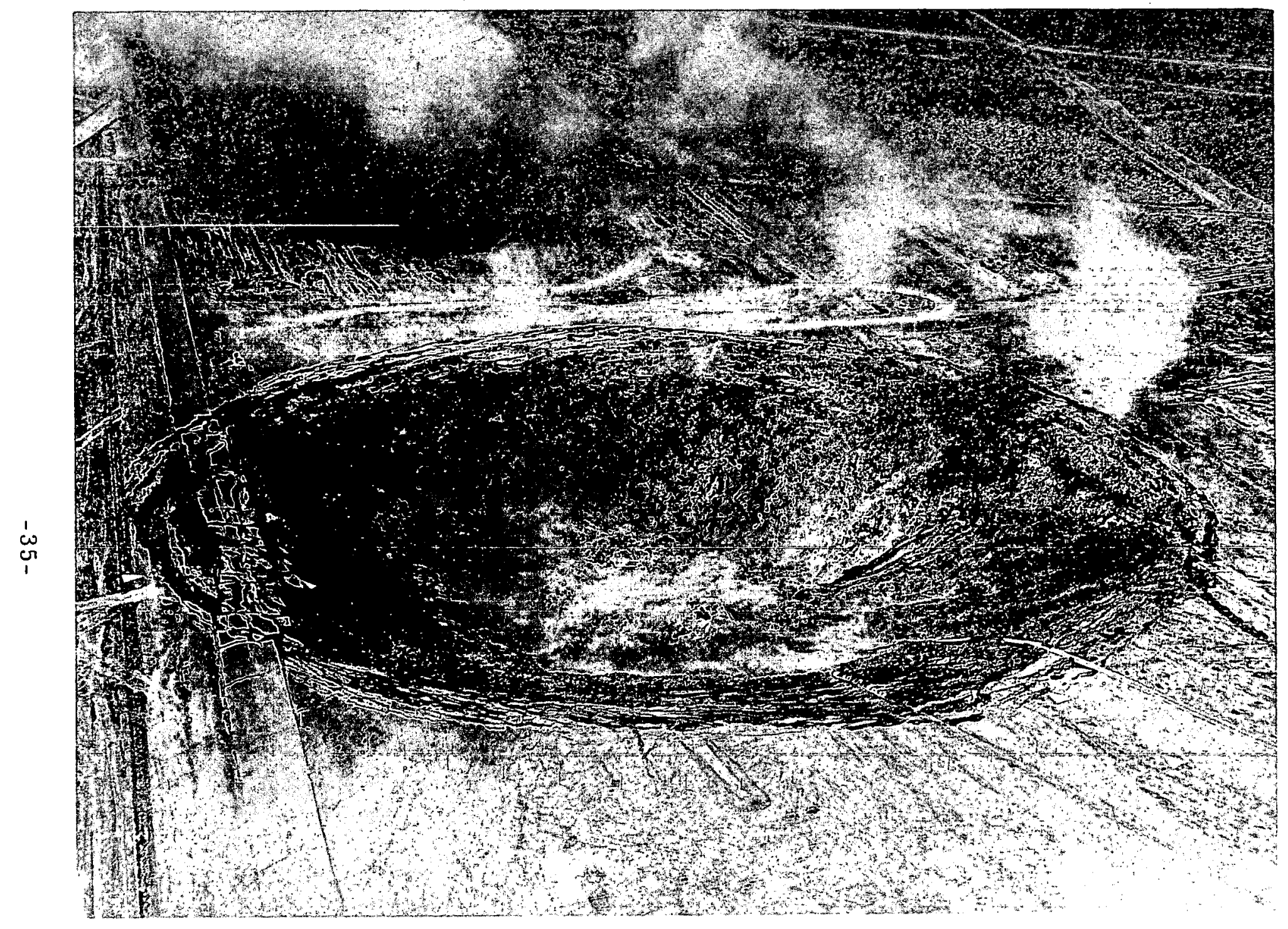

Figure 2.14 Subsidence crater. 


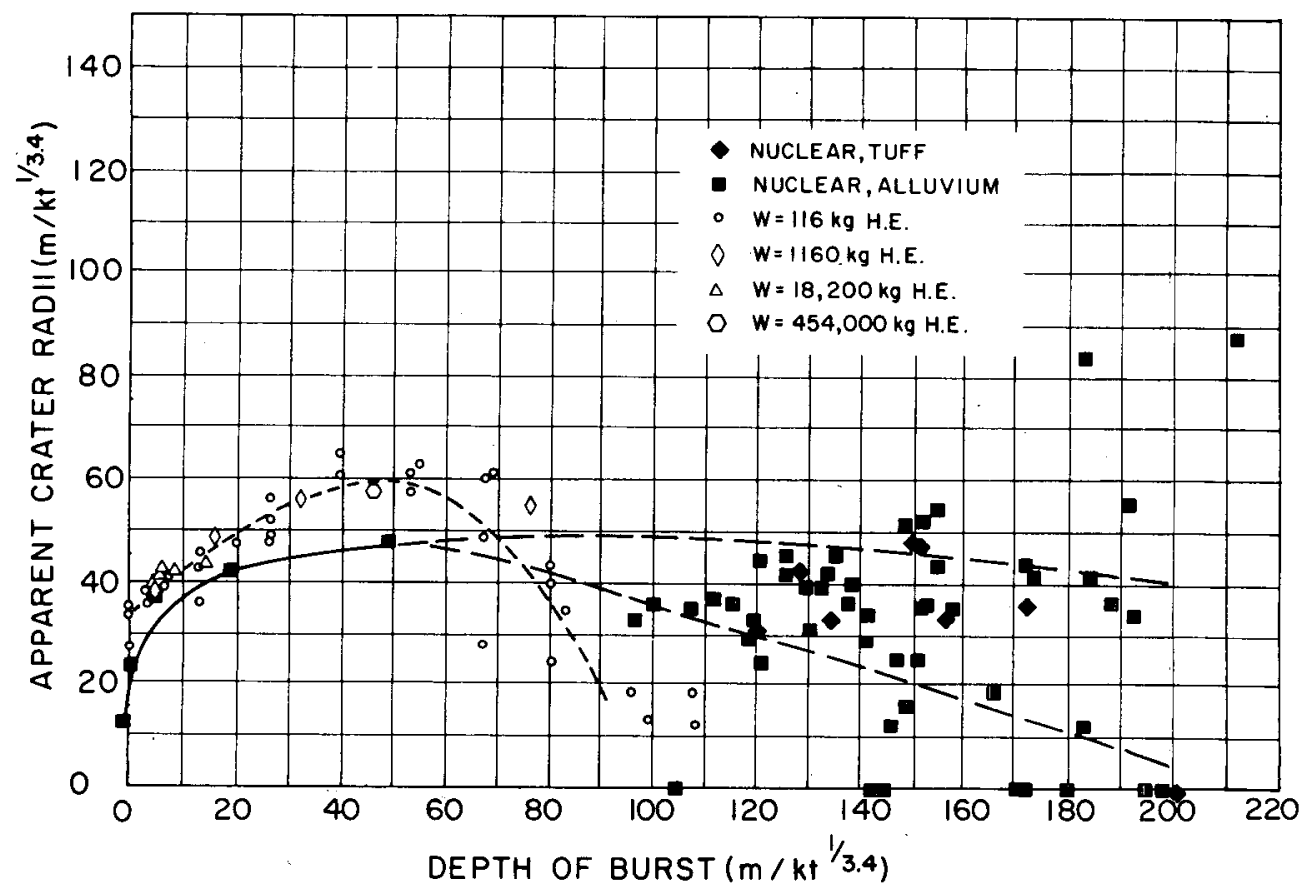

Figure 2.15 Apparent crater radius vs depth of burst.

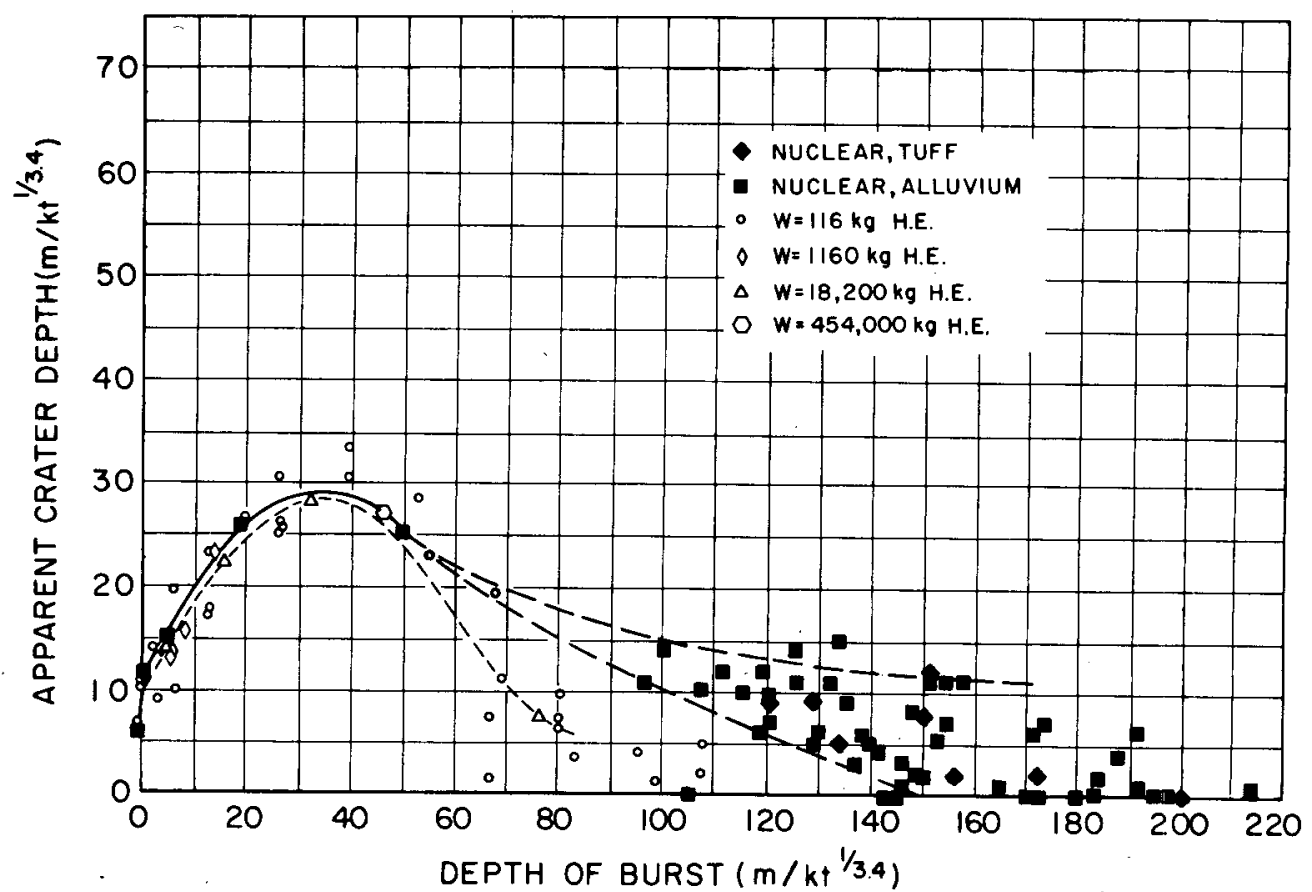

Figure 2.16 Apparent crater depth vs depth of burst. 
In some cases, for the subsidence craters, the detonation point was located in weakly cemented tuff, whereas in others it was located in alluvium. In all cases the major portion of the collapse chimney region was in alluvium. The nature of the detonation point medium has been indicated on the graphs.

Two special comments should be made regarding the applicability of the subsidence crater data. The first is that subsidence craters would only be expected in a medium such as desert alluvium where no bulking during collapse is observed. In a rock-type medium where bulking does indeed occur, the volume of the underground cavity would not be transmitted to the surface as it is in alluvium but would be distributed throughout the chimney region in the form of voids among the broken rock. In most rock media the collapse region would, in fact, not even reach the surface.

The second point to be made relates to the effect of yield. Chemical explosive experiments at the same scaled depths of burial as the nuclear subsidence craters resulted in very small craters or no craters. The presence of thin layers of caliche material with somewhat greater strength than the sand-gravel alluvium produced sufficient bulking and arching to stop any subsurface void from subsiding to the surface. . On the scale of the nuclear craters these layers are insignificant and the generalized nature of the alluvial material determined the behavior.

\subsubsection{Nuclear Cratering Curves for Alluvium: The above} data and considerations have led to the conclusion that the depthof-burst curves shown in Figures. 2.1 and 2.2 are not adequate for predicting nuclear craters in alluvium. On the basis of all available data, new curves for predicting nuclear crater dimensions have been drawn as shown by the solid curves in Figures 2.15 and 2.16. These recognize the reduced efficiency of nuclear explosives for apparent crater radii and assume $\mathrm{W}^{1 / 3.4}$ scaling. The curves 
for chemical explosives from Figures 2.1 and 2.2 are shown as dashed curves on Figures 2.15 and 2.16.

Because of the paucity of data it is not possible to establish any statistical limits on the nuclear curve, but based on past experience with the chemical explosive cratering curves it is estimated to be about \pm 10 percent on both radius and depth. At depths of burst greater than Sedan the curve is broadened into a zone that encompasses most of the subsidence data.

\subsection{CONCLUSIONS}

Comparison of the crater dimensions from Sedan with chemical explosive cratering experience and other nuclear explosive cratering experience in desert alluvium leads to the conclusions that:

a. Nuclear cratering explosions in the region of optimum depth of burst in alluvium generally result in craters with apparent radii about 10-20 percent smaller than equivalent yield chemical explosives. This corresponds to a chemical-nuclear explosive efficiency of about 50 percent for optimum depth of burial.

b. Apparent depths of nuclear explosive craters in alluvium are about equal to or slightly larger than equivalent-yield chemical explosive craters, corresponding to an efficiency for crater depth of about 100 percent.

c. Use of the above conclusions with $\mathrm{w}^{1 / 3.4}$ scaling derived from chemical explosive craters leads to a consistent picture for all available cratering data and permits establishment of independent nuclear explosive crater curves for scaled apparent crater radius and depth versus scaled depth of burst.

. The above conclusions are valid for yields up to $100 \mathrm{kt}$ with less than 10 percent error. Empirical scaling is inherently 
unreliable for extrapolation beyond the region of experience. However, from a consideration of the phenomena involved, no rapid changes in cratering phenomena are anticipated between 100 $\mathrm{kt}$ and $1 \mathrm{Mt}$ and the above conclusions are felt to be good estimates of expected behavior.

It must also be noted that the above conclusions are for alluvium and subsequent experiments in rock type mediums may lead to significantly different conclusions. 


\section{REFERENCES}

1. M. D. Nordyke, "An Analysis of Cratering Data from Desert Alluvium, "Journal of Geophysical Research, 1965-1974, 1962.

2. J. A. Chabai and D. M. Hankins, "Gravity Scaling Laws for Explosion Craters, "Sandia Corporation, SC-4541(RR), 1960.

3. W. R. Perret, and others, "Project Scooter, Final Report," Sandia Corporation, SC-4602(RR), 1963.

4. G. W. Johnson, "The Soviet Program for Industrial Applications of Explosives, " Lawrence Radiation Laboratory, UCRL-5932, 1960.

5. G. I. Pokrovskii and J. S. Fedorov, "Effect of Shock and Explosion on Deformable Media," Gas. IZd., 1957.

6. M. M. Dokuchaev, and others, "Handbook on Drill Blasting Operation in Construction, "Gas. IZd., 1962.

7. R. H. Carlson and W. A. Roberts, "Mass Distribution and Throwout Studies, Project Sedan, "The Boeing Company, PNE-217F, 1963.

8. W. D. Richards, "Geologic Study of the Sedan Nuclear Crater, Project Sedan, " Lawrence Radiation Laboratory, PNE-240F, June 1964.

9. E. Shoemaker, "Penetration Mechanics of High Velocity Nutrients, " International Geol. Congress, XXI, Part XVIII, 1960.

10. E. Shoemaker and R. Eggleton, "Terrestrial Features of Impact Origin, Proceedings of the Geophysical LaboratoryLawrence Radiation Laboratory Cratering Symposium, " Lawrence Radiation Laboratory, UCRL-6438 I, p. 1, 27, 1961.

11. W. E. Strohm, and others, "Stability of Crater Slopes, Project Sedan, "Waterways Experiment Station, PNE-234F, March 1964. 
12. M. D. Nordyke and W. R. Wray, "Cratering and Radioactivity Results from a Nuclear Cratering Detonation in Basalt, Project Danny Boy, "Lawrence Radiation Laboratory, UCRL-6999 Rev. II, October 1963. 


\section{CHAPTER 3}

\section{FALLOUT STUDIES}

\subsection{SUMMARY OF PAST EXPERIENCE}

3.1.1 General: Previous work ${ }^{1-6}$ with chemical and nuclear explosives has shown that cloud development and fallout distribution from an underground detonation differ in several ways from surface and atmospheric explosions.

First, only a small portion of the radioactivity generated in a deeply buried explosion is deposited as local fallout. Most of the radioactivity is scavenged by material which falls back into the crater and on the crater lip. The results of past work show that one should expect some 1-10 percent of the activity to escape from the crater and appear as local fallout when firing at the depths of burst of interest in nuclear excavation. Much of the activity which escapes is carried on the large dust particles which fall within a relatively short distance of ground zero.

Secondly, the height of the main cloud decreases considerably as the explosive is buried deeper and deeper. For depths of burst slightly deeper than optimum for cratering, the cloud height may be only 10-25 percent of that expected from a surface burst of the same yield. Also, as the fallback material and ejecta fall through the air and hit the ground surface, a large base surge cloud is formed which spreads a large portion of the vented radioactivity over a relatively small area surrounding the crater. The lower cloud height and the base surge phenomenon are largely responsible for deposition of most of the local fallout at comparatively short ranges for deeply buried cratering explosions.

\subsubsection{Relationship of Sedan to Past Nuclear Cratering}

Events: Prior to Sedan, there had been five nuclear events fired at the Nevada Test Site which resulted in large craters and are, therefore, of interest in the problem of understanding radioactivity 
resulting from nuclear cratering explosions. Three of these, Jangle Surface, Jangle Underground, and Teapot ESS were weapon effects tests fired in desert alluvium at depths of burst much shallower than those proposed for Plowshare applications. A fourth event, Neptune, was fired in bedded tuff on the Rainier Mesa at very deep burial. The fifth, Danny Boy, was fired near optimum depth for cratering in the basalt of Buckboard Mesa. Yields range from 115 tons to $1.2 \mathrm{kt}$ for these events. The explosives were all-fission devices.

Sedan had as one of its primary objectives the study of the release and distribution of radioactivity from an explosive in the 100 -kt range detonated at a depth slightly greater than optimum for cratering. Sedan offered comparis on with Danny Boy to show yield and shot-medium effects on radioactivity release and distribution; with Jangle Surface, Jangle Underground and Teapot ESS to show yield and depth of burial effects; and with Neptune to show yield, shot medium and depth of burial effects on the radioactivity problem.

\subsection{PREDICTIONS}

3.2.1 Activity Production: The amount of radioactivity produced in a nuclear cratering explosion is dependent upon the fission yield of the explosive, the neutron fluxes available for creating induced radioactivities and the types of materials in the explosive and surrounding the explosive which will absorb these neutrons.

Previous tests with devices similar to the Sedan device gave a basis for prediction of the fission yield, the activation of device components and the neutron flux available for soil activation.

These predictions showed the only significant activation of device materials to be expected was from tungsten. 
Prediction of soil activation was based on a chemical analysis of the shot medium and theoretical calculations of the neutron flux into the soil. - These calculations recognized the neutron shielding in and around the explosive, and used a soil moisture content of 3.2 percent. This moisture content is felt to be low by a factor of four to five compared to the probable water content at depth. Thus the results of these calculations gave a maximum credible yield of soil-induced activities. The largest contributor to the soil activation was expected to be $\mathrm{Na}^{24}$, accounting for 85 percent of the infinite dose attributed to soil-induced activities.

The amount of radioactivity which comes down as local fallout is dependent upon the depth of burial of the explosive and on the type of material in which the explosive is detonated. Results of the previous cratering events were used as a basis to predict the fraction of the radioactivity produced that would be expected to be deposited in the Sedan fallout pattern. On this basis, the amount of radioactivity in the Sedan fallout pattern from all of the above sources was predicted to be equivalent in terms of the infinite dose from one hour, to $0.42 \mathrm{kt}$ of fission-product activity.

3.2.2 Cloud Dimensions: Cloud dimensions were predicted using a theoretical approach to cloud scaling suggested by J. B. Knox ${ }^{7}$ and using Teapot ESS as a scaling base. The Sedan cloud was predicted to have the following dimensions:

$\begin{array}{lcc} & \text { Height }(\mathrm{m}) & \text { Radius }(\mathrm{m}) \\ \text { Main Cloud } & 1200 & - \\ \text { Base Surge } & 600 & 4900\end{array}$

These predictions assumed a statically stable atmosphere. Since the prediction of cloud height was based on limited experience, the cloud height was arbitrarily doubled to 2400 meters for the fallout calculations. 
3.2.3 Fallout Distribution: The fallout prediction ${ }^{8}$ was based on A. V. Shelton's cloud model and fallout prediction system. To convert calculated fallout densities to external gamma field readings conversion constants of $9700 \mathrm{R} / \mathrm{hr}$ and $32,400 \mathrm{R}$ were used as the $\mathrm{H}+1$ hour dose rate and infinite dose from one hour, respectively, 1 meter above an infinite plane surface uniformly contaminated by fission products at a rate of $1 \mathrm{kt} / \mathrm{km}^{2}$. A terrain shielding factor of 0.7 was assumed. A set of typical winds was assumed as follows:

\begin{tabular}{ccc} 
Altitude $(\mathrm{m})$ & Speed $(\mathrm{km} / \mathrm{hr})$ & Direction $\left(^{\circ}\right)$ \\
\hline $0-300$ & 15 & 220 \\
$300-900$ & 18.5 & 210 \\
$900-2400$ & 22 & 190
\end{tabular}

Results of the prediction are shown in Figure 3.1 as infinite dose as a function of distance along the hot line.

\subsection{RESULTS}

The major programs involved in the Sedan fallout studies included meteorological observations, cloud photography to study cloud development, complete ground and aerial surveys to establish fallout patterns, radiochemical analysis of cloud and fallout samples, and particle-size analysis of fallout samples. Radiochemical and particle-size analyses are given in more detail elsewhere. 9,10

\subsubsection{Meteorology: A fairly complete set of meteorological} observations was made and reported by the USWB. ${ }^{11}$ Only a temperature-vs-altitude plot of shot-time conditions is shown here (Figure 3.2).

3.3.2 Cloud Development: The base surge formation became apparent at about 10-15 seconds and continued to grow to a maximum radius of about $4.0 \mathrm{~km}$ cross-wind, $2.5 \mathrm{~km}$ upwind, and 


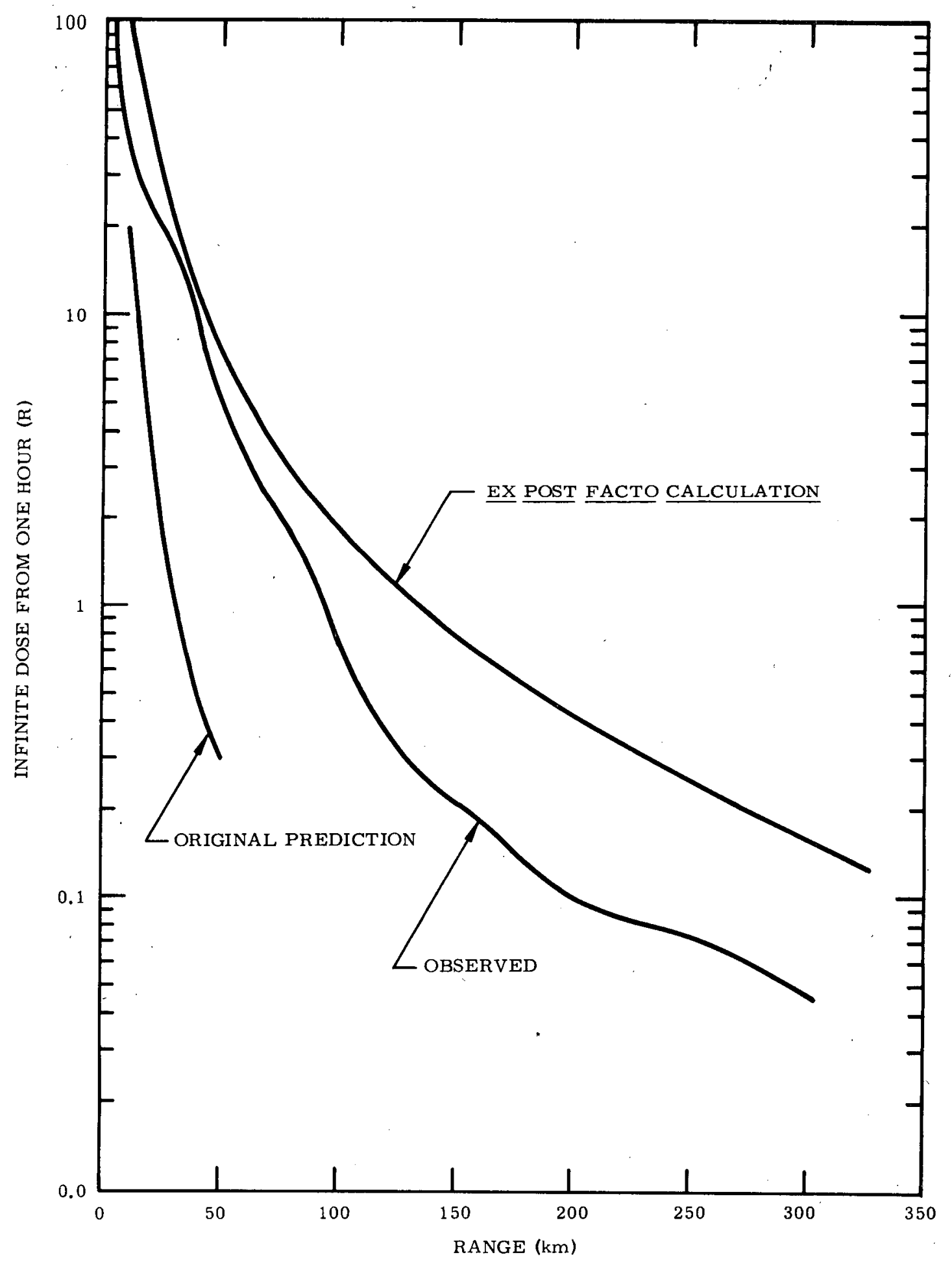

Figure 3.1 Comparis on of observed dose along the hotline to the original prediction and the ex post facto calculation. 


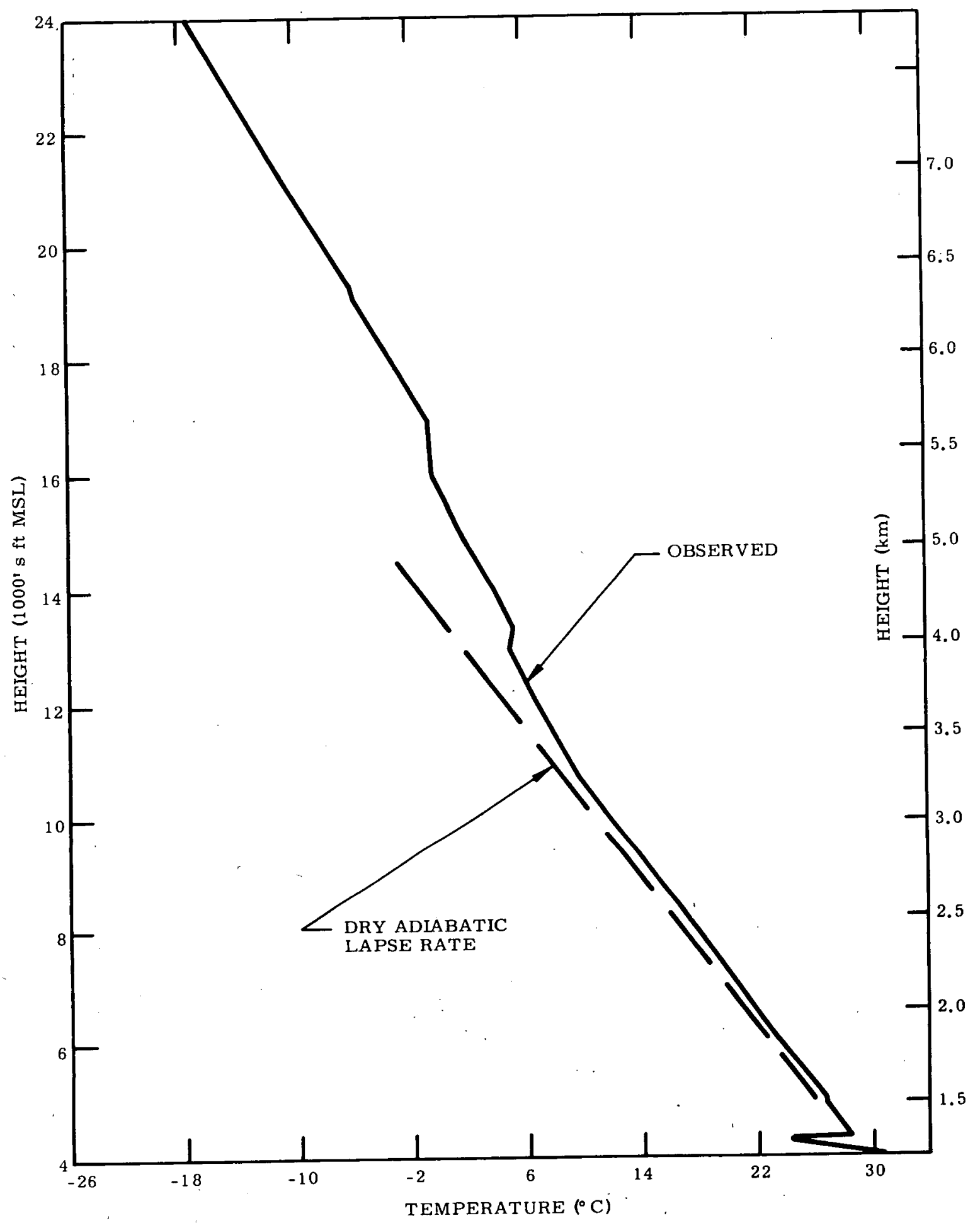

Figure 3.2 Temperature profile, Yucca weather station, 1022 PDT, GZ elevation $4317 \mathrm{ft}$ MSL. 
a height of about 1200 meters. ${ }^{12}$ The main cloud rose to a height of about 3600 meters $^{11}$ above the surface where it was apparently stopped by a very stable layer. The main cloud reached a diameter of about 1800 meters before the winds began to spread it and move it on downwind. The cloud appeared to travel on a bearing of $\mathrm{N} 0-5^{\circ} \mathrm{E}$ for the first $40-80 \mathrm{~km}$, then turned easterly at a bearing of $\mathrm{N} 20-25^{\circ}$ E out to a range of $240-320 \mathrm{~km}$. The cloud traveled at a speed of about $27 \mathrm{~km} / \mathrm{hr}$. The last visual observation of the cloud was near Ely, Nevada, about $250 \mathrm{~km}$ away. At this time, the cloud was $55-65 \mathrm{~km}$ wide. 13

\subsubsection{Discussion of Cloud Development: The Sedan cloud} rose to a height considerably greater than predicted. This was partly a result of a neutral atmosphere existing to nearly 2700 meters. The stable layers from 2700 to 3600 meters apparently retarded the cloud rise and the very stable layer (nearly isothermal) from 3600 to 3900 meters almost completely stopped the cloud rise (Figure 3.2). Another possible reason for the large cloud was the relatively high moisture content of the soil (10-20\%) at shot depth which produced a large volume of gas at detonation. The prediction was based on a stable atmosphere and on the Teapot ESS event which probably had a lower soil moisture content and consequently less gas produced per unit yield.

\subsubsection{Radiation Field Measurements: On-site monitor} surveys commenced at about $\mathrm{H}+1$ hour and continued at varying intervals through D+33 days. Earliest readings on the crater lip were made on $\mathrm{D}+27$ days and again on $\mathrm{D}+40$ days. Crater entry was made on $\mathrm{D}+167$ days, at which time the maximum reading in the bottom of the crater was $35 \mathrm{mR} / \mathrm{hr}^{14}$

A remote-area monitoring system was used on-site to obtain early high intensity radiation readings. Much of the data are unreliable, however, due to instrument malfunctions, instrument 
contamination and radiation fields which exceeded the range of some instruments.

Off-site monitoring began on $\mathrm{D}$ day but some of the data taken were considered unreliable due to contamination of vehicles and instruments. A complete remonitoring of the off-site area was performed on $\mathrm{D}+1$ day. At a few locations expected to be in the cloud path, the residents were relocated during the period of cloud passage. At several locations the residents remained indoors during cloud passage. All residents in areas expected to receive radiation were equipped with film badges. The highest exposure dose recorded was $275 \mathrm{mR}$. However, two other residents at the same location had exposure doses of 85 and $90 \mathrm{mR}$ recorded on their film badges and it is possible that some of the $275-\mathrm{mR}$ exposure was due to heat damage. ${ }^{13}$

\subsubsection{Pattern Construction: The bulk of the most reliable} and comprehensive radiation survey data were taken from about $\mathrm{H}+20$ hours to $\mathrm{H}+28$ hours. For this reason the fallout patterns were constructed to show dose rates at $\mathrm{H}+24$ hours (Figure 3.3 and 3.4). This procedure minimized errors caused by using $\mathrm{t}^{-1.2}$ decay since, in general, the readings were decayed no more than about 4 hours. Observed decay during this time period did not depart markedly from $\mathrm{t}^{-1.2}$ so that the method probably gave fair treatment of the data. The edge of the pattern in the base surge area was, in general, drawn to conform to the area delineated by the dust deposit as shown in a photo mosaic of the Sedan area developed by NRDL ${ }^{15}$ (Figure 3.5).

\subsubsection{Discussion of Pattern Formation: The pattern shape} shows the effect of the terrain on the wind structure. The wind appears to have carried the cloud preferentially along the valleys and to have been retarded by the mountain ranges. Best evidence for this is along the west side of the pattern where the Kawich Range held the cloud generally in Reveille and Railroad Valleys 


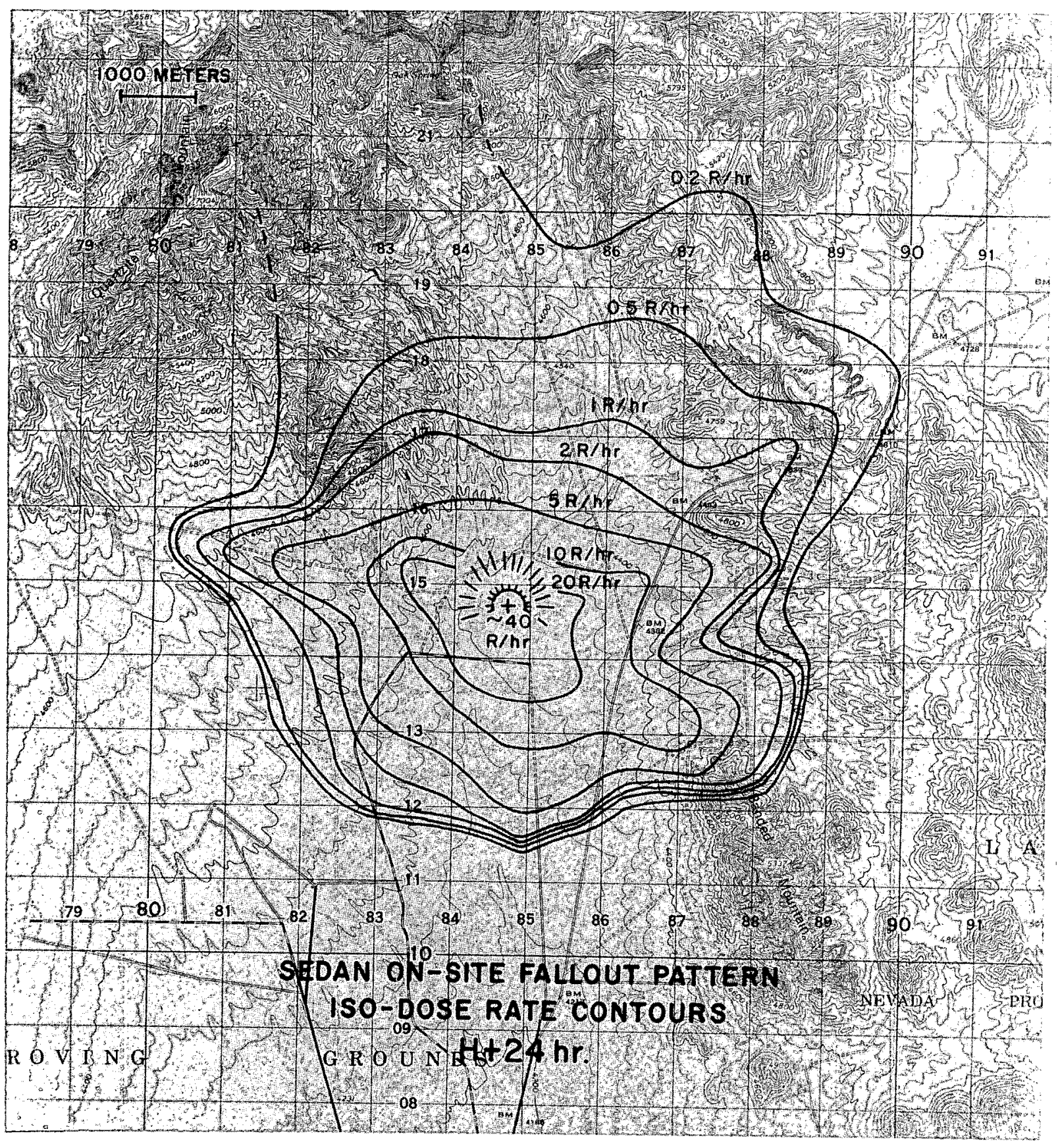

Figure 3.3 Sedan on-site fallout pattern, isodose rate contours, $\mathrm{H}+24$ hours. 


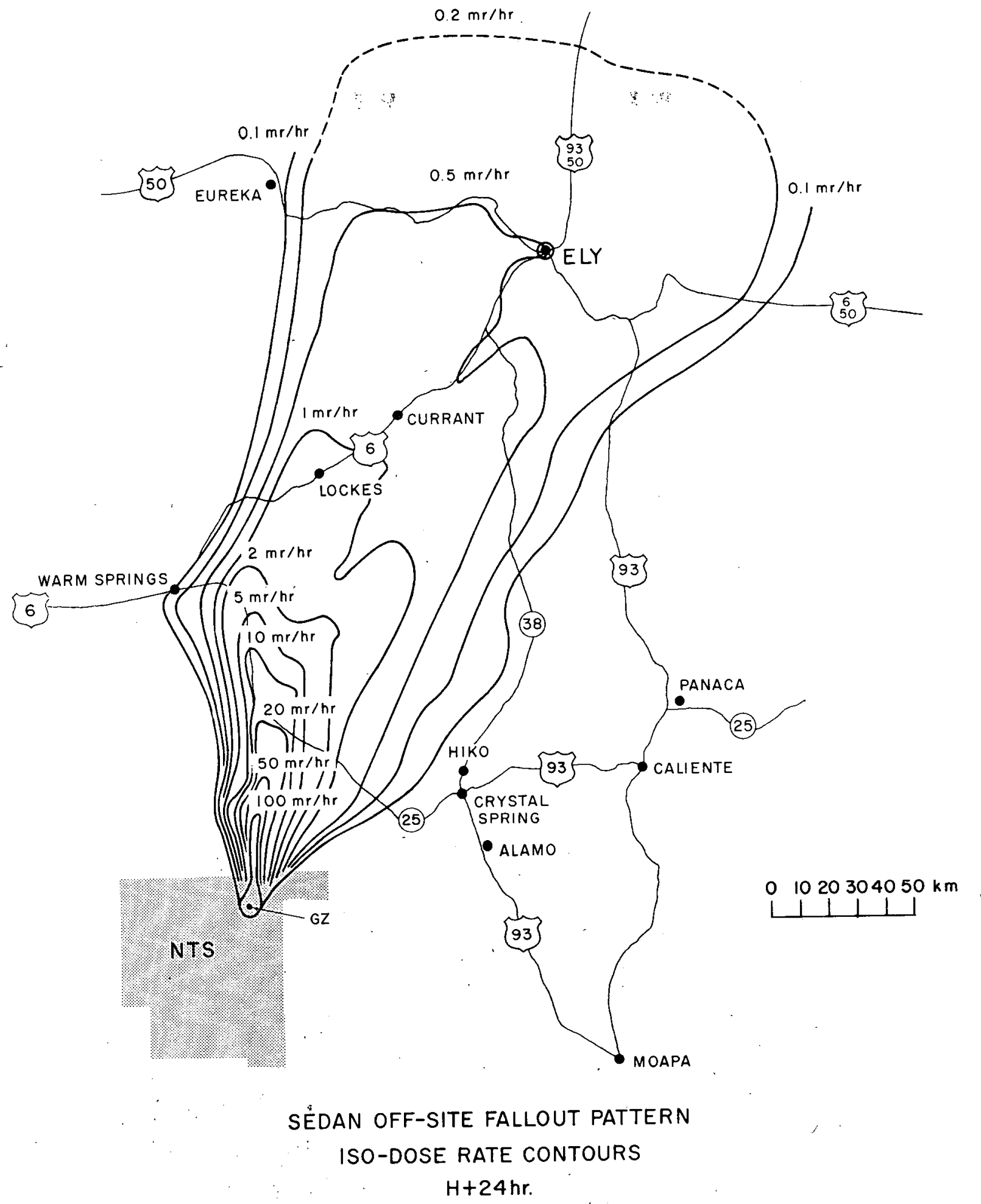

Figure 3.4 Sedan off-site fallout pattern, isodose rate contours, $\mathrm{H}+24$ hours. 


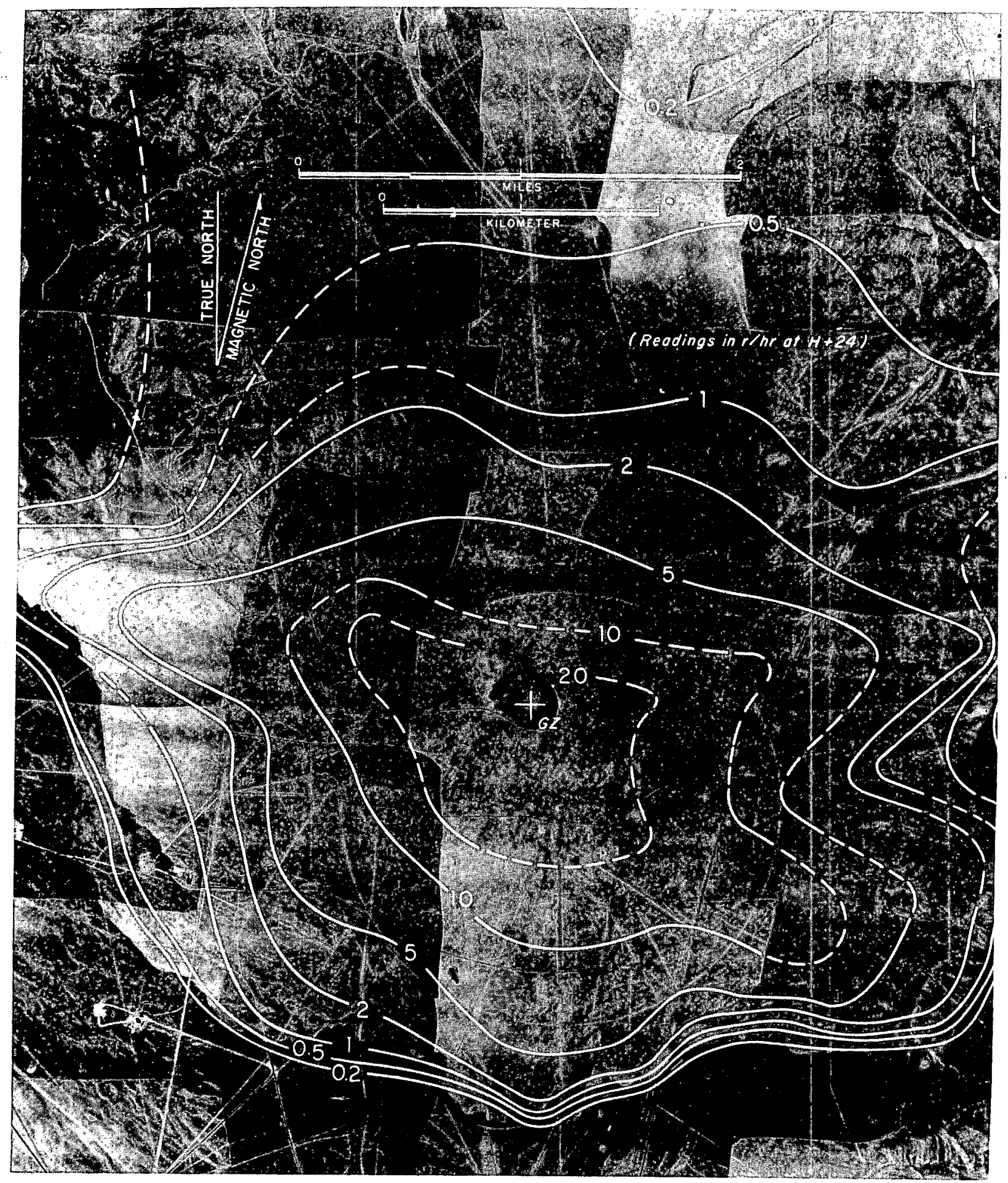

Figure 3.5 Aerial photomosaic showing base surge deposition and on-site fallout pattern. 
to Warm Springs. There the cloud was turned by the Hot Creek Range and Antelope Range and continued on up Railroad Valley. The necking in the center of the pattern was probably caused in part by the Grant Range.

The winds at about 75 percent of the base surge height should reflect the fallout from the base surge and lower levels of the main cloud. This is supported by the direction of the initial hot line which conforms to the direction of the effective wind at 75 percent of base surge height.

The effective wind at 75 percent of main cloud height was in a direction about $33^{\circ}$ east of north with almost $33^{\circ}$ shear from 75 percent of base surge height to 75 percent of main cloud height so that the top of the cloud was spread to the east. This gave an effect of spreading the activity so that no real hot line developed, but rather a gradual gradient appeared on the east. This is in contrast to the lower layers where almost no shear existed giving rise to a sharp hot line along the western edge. The upper levels of the cloud were high enough so that terrain canalization was not particularly in evidence on the east side of the pattern.

Certainly, such an analysis using the shot time winds cannot be applied throughout the length of the pattern since one would expect significant changes in the wind structure in a distance of 160 meters and a time span of 5-6 hours.

\subsubsection{Pattern Analysis and Comparison to Predictions:} Radiochemistry results 16,17 show that about 42 percent of the $\mathrm{H}+24$ hour dose rate was due to fission-product activity, approximately 55 percent due to $\mathrm{W}^{187}$, approximately 2 percent due to $\mathrm{Na}^{24}$ and $<1$ percent due to activation of soil and device materials, tracers, and thermonuclear products. These ratios assume no fractionation, and that like fractions of all activities escape from the crater. Variation of the dose rate from each activity, 
component with time is shown in Figure 3.6. The curves show that the $\mathrm{W}^{187}$ and $\mathrm{Na}^{24}$ are decayed to negligible levels by $\mathrm{D}+10$ days. The nuclides $\mathrm{Sc}^{46}, \mathrm{Sc}^{44 \mathrm{~g}}, \mathrm{Sc}^{44 \mathrm{~m}}, \mathrm{Y}^{88}$ and $\mathrm{Rh}^{102}$ are radiochemistry tracers added to the device for diagnostic purposes. $\mathrm{Be}^{7}$ is a fusion reaction product.

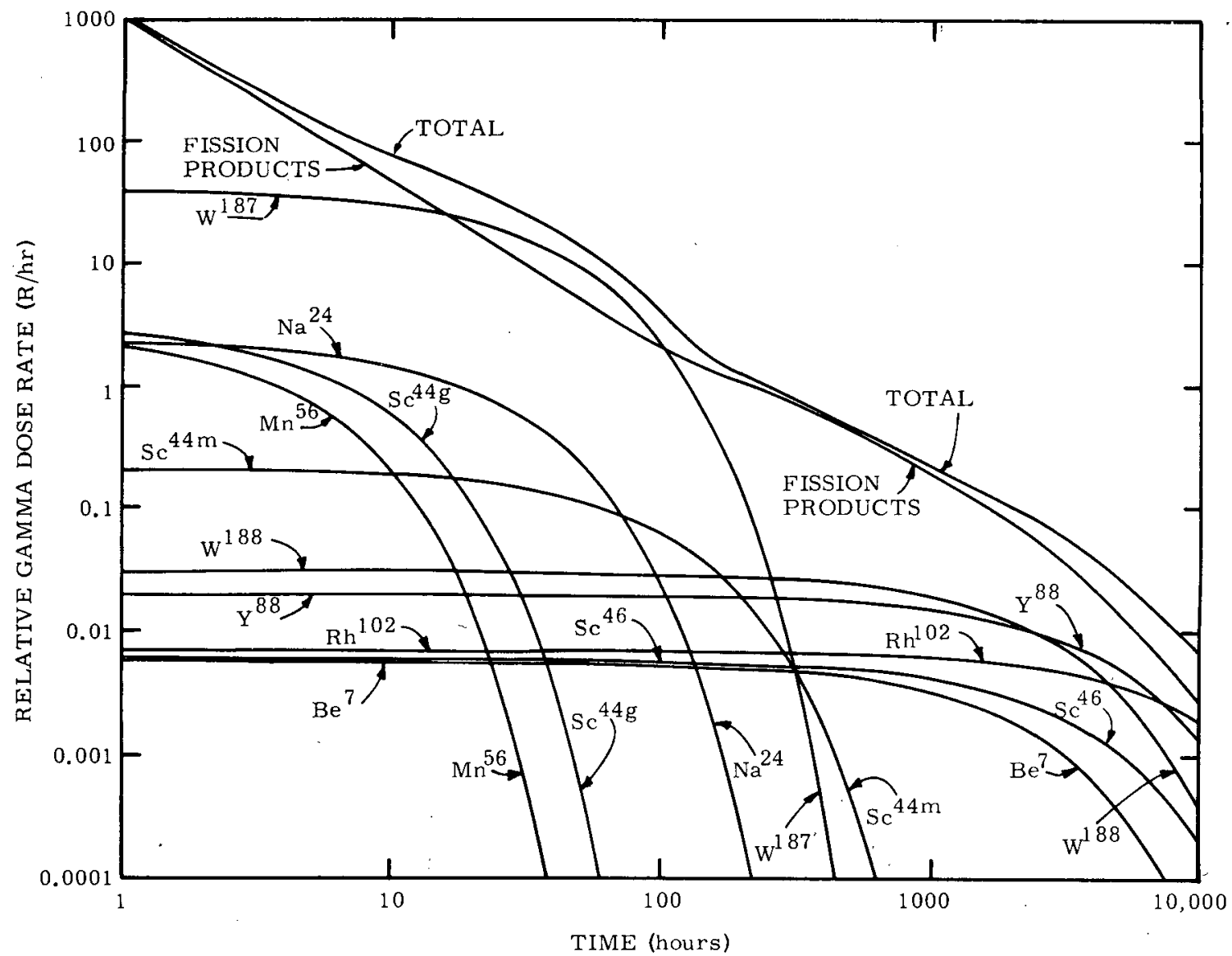

Figure 3.6 Unfractionated debris decay showing relative dose rates from important gamma emitters between 1 and 10,000 hours after zero time. 
The fallout pattern was integrated to determine the amount of radioactivity deposited in the fallout pattern. ${ }^{18}$ This integration gives an infinite dose from $\mathrm{H}+1$ hour equivalent to $2.0 \mathrm{kt}$ of fissionproduct activity. This is greater than expected by a factor of nearly five. This difference is due in large part to the uncertainty in extrapolating the results of the previous events to the depth of burst at which Sedan was fired. Only the Danny Boy event had a scaled depth of burst near that of Sedan and it was fired in a different shot medium.

The pattern was truncated at various ranges and the truncated patterns were integrated. These integrations were compared to the total integration to show the fraction of the total activity which is deposited as a function of time or distance. An average wind speed was used to calculate an average arrival time at each range. The results of these calculations were then plotted to show the fraction of the total activity remaining airborne as a function of time (Figure 3.7). This curve shows that most of the activity was deposited at very early times. A similar calculation shows that 70 percent of the total activity in the fallout pattern was deposited inside the area of the initial base surge.

Certainly some of the difference between predictions and measurements must derive from the uncertainties in determining fission yield and the amounts of various components of the fallout samples. There may also be a significant error in assuming no fractionation and no enrichment or depletion of various activities during the venting process, although preliminary data indicate no significant fractionation in the Sedan debris. ${ }^{10}$

A gross approximation of terrain shielding can be made using preliminary radiochemical analyses of five fallout tray samples. ${ }^{17}$ These calculations show an average activity density for the five stations, of $219 \pm 97(\mathrm{R} / \mathrm{hr}) /\left(\mathrm{kt} / \mathrm{km}^{2}\right)$ at $\mathrm{H}+24$ hours (Table 3.1). This can be interpreted as the external dose rate one 


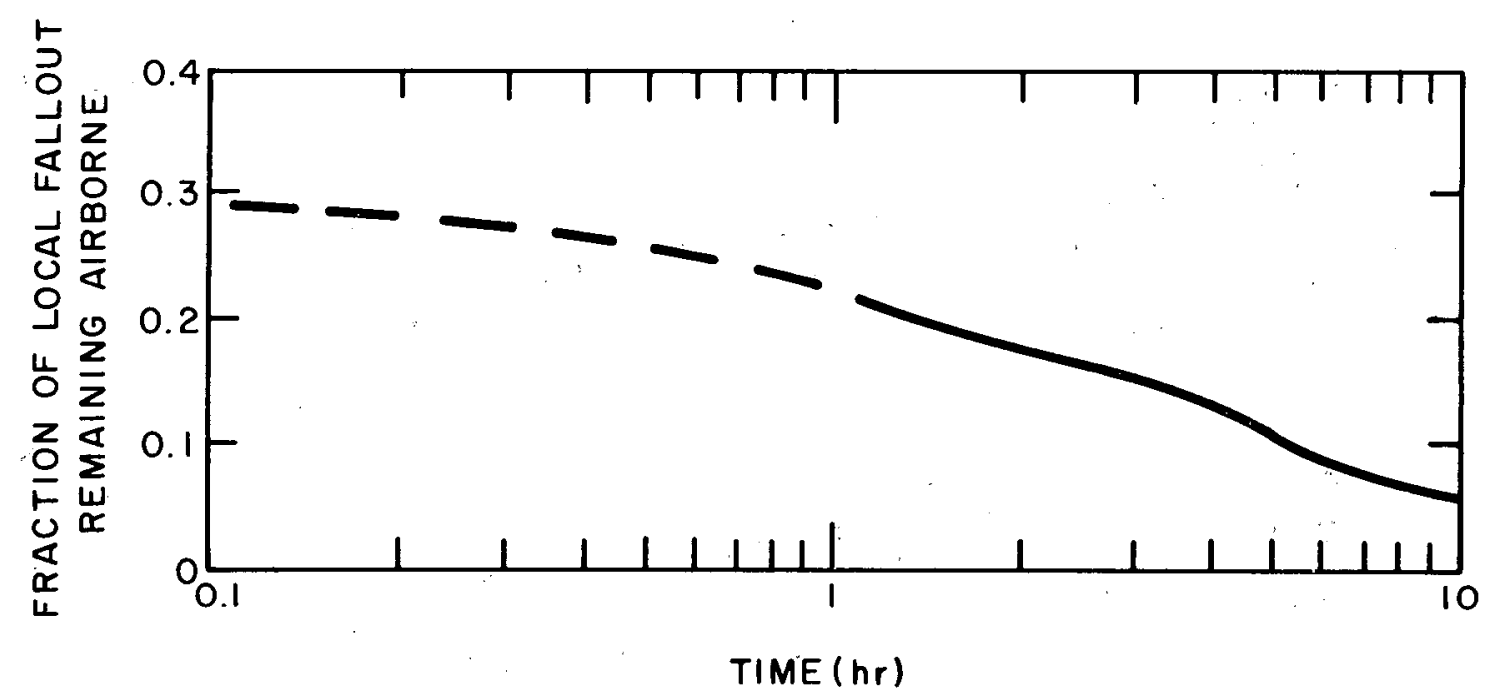

Figure 3.7 Fraction of local fallout remaining airborne as a function of time.

TABLE 3.1. ACTIVITY DENSITY DETERMINED FROM FALLOUT TRAY SAMPLES

\begin{tabular}{llccc}
\hline Station & $\begin{array}{l}\text { Fissions } \\
\text { per tray }\end{array}$ & $\begin{array}{l}\text { Fission } \\
\text { density }\end{array}$ & $\begin{array}{c}\text { Dose rate } \\
\text { at } \mathrm{H}+24 \mathrm{hr}\end{array}$ & $\begin{array}{c}\text { Activity } \\
\text { density }\end{array}$ \\
\hline & & $\left(\mathrm{kt} / \mathrm{km}^{2}\right)^{\mathrm{a}}$ & $(\mathrm{R} / \mathrm{hr})$ & $(\mathrm{R} / \mathrm{hr}) /\left(\mathrm{kt} / \mathrm{km}^{2}\right)$ \\
B-11 & $1.46 \times 10^{14}$ & $1.38 \times 10^{-2}$ & 3.6 & 261 \\
D-5 & $3.0 \times 10^{13}$ & $2.84 \times 10^{-3}$ & 0.243 & 86 \\
K-16 & $1.41 \times 10^{13}$ & $1.34 \times 10^{-3}$ & 0.332 & 248 \\
P-11 & $2.38 \times 10^{14}$ & $2.26 \times 10^{-2}$ & 4.4 & 195 \\
Q-5 & $1.22 \times 10^{14}$ & $1.16 \times 10^{-2}$ & 5.1 & 440 \\
J-10.5 & $1.38 \times 10^{13}$ & $1.31 \times 10^{-3}$ & 0.111 & Av. $=\frac{85}{219} \pm 97$ \\
& & & & (R/hr) $/\left(\mathrm{kt} / \mathrm{km}^{2}\right)$ \\
\hline
\end{tabular}

$\mathrm{a}_{\text {Tray area }}=7.54 \times 10^{-8} \mathrm{~km}^{2} ; 1 \mathrm{kt}=1.4 \times 10^{23}$ fission events. 
would have measured at $\mathrm{H}+24$ hours one meter above the Sedan terrain, if it had been uniformly contaminated with Sedan debris at a rate of $1.4 \times 10^{23}$ fissions (i. e. $1 \mathrm{kt}$ ) per square kilometer. The corresponding theoretical dose rate one meter above an infinite smooth plane is calculated to be $308(\mathrm{R} / \mathrm{hr}) /\left(\mathrm{kt} / \mathrm{km}^{2}\right)$. This calculation was made using the method of Higgins 19 and the actual amounts of induced radionuclides produced, and assumes unfractionated fission products. Thus the average correction factor for conversion of infinite smooth-plane dose rates to field readings is approximately 0.7 for terrain such as Sedan. It should be pointed out, however, that this correction factor is also dependent on fractionation and would therefore be invalid for an event in which the vented debris is fractionated in a manner significantly different from Sedan.

An effort was made to correlate fallout mass deposition with radiation intensity in the initial base surge area. The mass density was calculated from the mass collected in fallout trays located at each of 46 stations between 1.5 and $6.5 \mathrm{~km}$ from surface zero (Figure 3.8, Table 3.2). The mass per unit area per unit dose rate for each station was then calculated using the mass density and the measured dose rate decayed to $\mathrm{H}+24$ hours. The average for the 46 stations is $1.53 \pm 0.67\left(\mathrm{~kg} / \mathrm{m}^{2}\right) /(\mathrm{R} / \mathrm{hr})$. As the depth of fallout deposition increases beyond a centimeter or more such a correlation becomes increasingly invalid due to selfshielding by the deposited material.

\subsection{DISCUSSION}

3.4.1 Radioactivities: Since the time that the original Sedan predictions were made, independent developments have led to changes in predictive techniques and constants used in the prediction system. Using present techniques ${ }^{18}$ and constants ${ }^{2}$ one would predict an infinite dose from zero time for $\mathrm{W}^{187}$ which is 


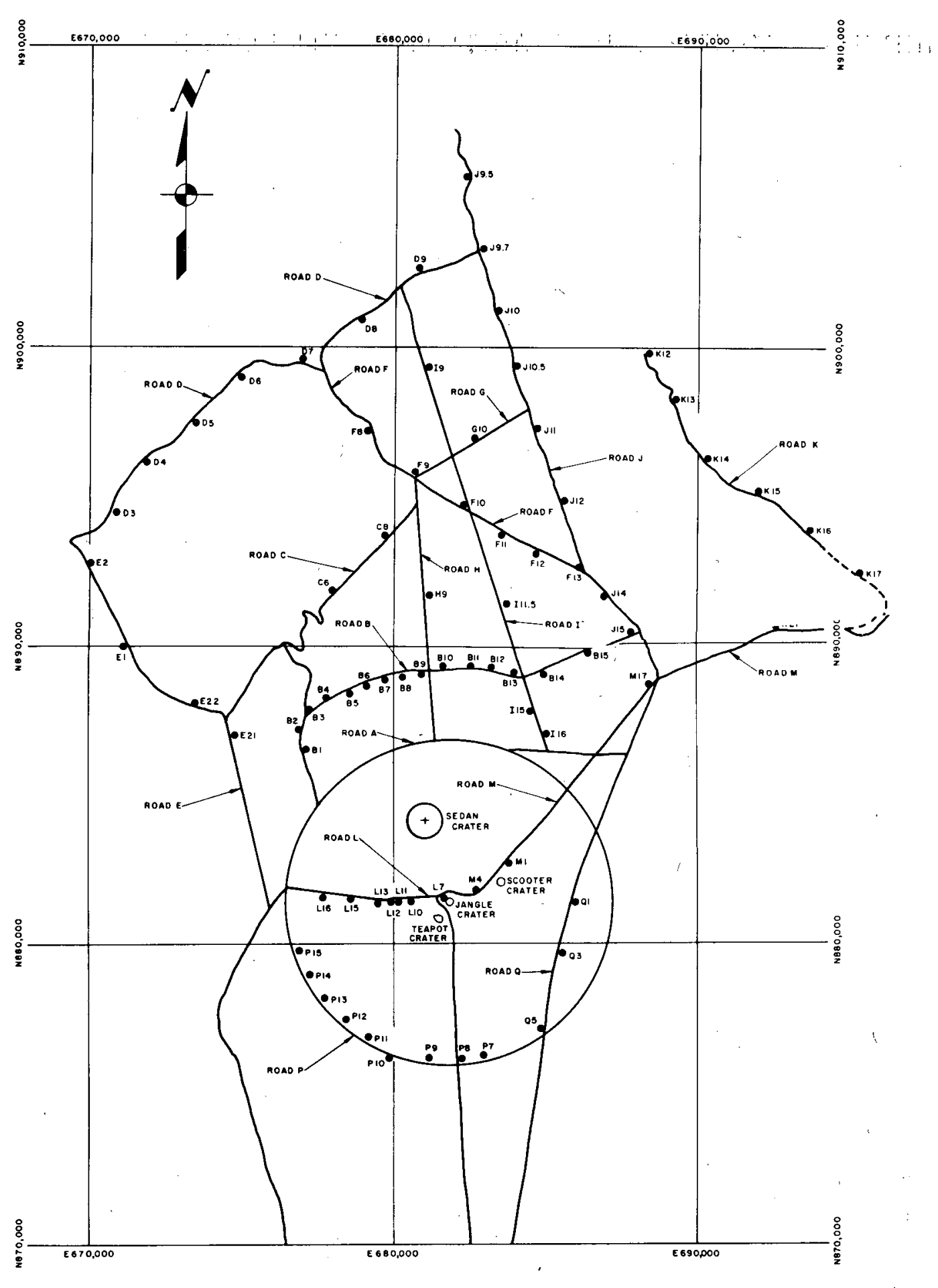

Figure 3.8 Fallout station array, Project Sedan. 
TABLE 3.2 MASS PER UNIT AREA PER UNIT DOSE RATE AT VARIOUS FALLOUT STATIONS

\begin{tabular}{|c|c|c|c|c|}
\hline Station & $\begin{array}{c}\text { Weight } \\
\text { Collected }\end{array}$ & $\begin{array}{c}\text { Gamma } \\
\text { Dose Rate }\end{array}$ & $\begin{array}{l}\text { Time of } \\
\text { Yield } \\
\text { Reading }\end{array}$ & $\begin{array}{c}\text { Mass per } \\
\text { Unit Area } \\
\text { per Unit } \\
\text { Dose Rate } \\
\end{array}$ \\
\hline & $(\mathrm{kg})$ & $(\mathrm{R} / \mathrm{hr})$ & (hrs after $\mathrm{H} h r$ ) & $\left(\mathrm{kg} / \mathrm{m}^{2} / \mathrm{R} / \mathrm{hr}\right)$ \\
\hline B-6 & 0.802 & 4.4 & 28.25 & 2.16 \\
\hline-10 & 0.463 & 3.0 & 28 & 1.83 \\
\hline-11 & 0.454 & 3.2 & 28 & 1.67 \\
\hline-12 & 0.346 & 2.4 & 28 & 1.70 \\
\hline-13 & 0.228 & 1.5 & 27.75 & 1.78 \\
\hline-14 & 0.327 & 2.4 & 28 & 1.60 \\
\hline-15 & 0.231 & 1.0 & 28 & 2.76 \\
\hline$C-6$ & 0.060 & 1.75 & 26.25 & 0.41 \\
\hline-8 & 0.103 & 0.6 & 26.25 & 2.08 \\
\hline$D-3$ & 0.002 & 0.175 & 25.25 & 0.14 \\
\hline-4 & 0.0025 & 0.03 & 25.5 & 1.04 \\
\hline-5 & 0.015 & 0.225 & 25.75 & 0.82 \\
\hline-6 & 0.021 & 0.365 & 25.75 & 0.70 \\
\hline-7 & 0.028 & 0.350 & 25.75 & 0.99 \\
\hline-8 & 0.0096 & 0.10 & 26 & 1.06 \\
\hline$F-8$ & 0.070 & 0.225 & 26 & 3.79 \\
\hline-9 & 0.095 & 0.350 & 26.25 & 3.30 \\
\hline-11 & 0.070 & 0.500 & 27.25 & 1.67 \\
\hline-12 & 0.090 & 0.180 & 50.5 & 2.61 \\
\hline-13 & 0.093 & 0.800 & 27.25 & 1.39 \\
\hline$G-10$ & 0.020 & 0.100 & 26.5 & 2.33 \\
\hline $\mathrm{H}-9$ & 0.087 & 0.025 & 26.4 & 2.61 \\
\hline$I-9$ & 0.034 & 0.150 & 27 & 2.71 \\
\hline-11.5 & 0.108 & 0.220 & 50.5 & 2.57 \\
\hline-16 & 0.672 & 1.6 & 50.5 & 2.16 \\
\hline$J-9.5$ & 0.0096 & 0.100 & 27 & 1.15 \\
\hline
\end{tabular}


TABLE 3.2 (Continued)

\begin{tabular}{|c|c|c|c|c|}
\hline Station & $\begin{array}{c}\text { Weight } \\
\text { Collected }\end{array}$ & $\begin{array}{c}\text { Gamma } \\
\text { Dose Rate }\end{array}$ & $\begin{array}{l}\text { Time of } \\
\text { Field } \\
\text { Reading }\end{array}$ & $\begin{array}{l}\text { Mass per } \\
\text { Unit Area } \\
\text { per Init } \\
\text { Dose Rate } \\
\end{array}$ \\
\hline & (kg) & $(\mathrm{R} / \mathrm{hr})$ & (hrs after $\mathrm{H} \mathrm{hr}$ ) & $\left(\mathrm{kg} / \mathrm{m}^{2} / \mathrm{R} / \mathrm{hr}\right)$ \\
\hline$J-9.75$ & 0.0083 & 0.026 & 50.5 & 1.66 \\
\hline-10.5 & 0.013 & 0.100 & 26.75 & 1.56 \\
\hline-11 & 0.042 & 0.400 & 27 & 1.26 \\
\hline-12 & 0.0705 & 0.150 & 50.5 & 2.44 \\
\hline-15 & 0.116 & 0.600 & 27 & 2.33 \\
\hline-16 & 0.0766 & 0.800 & 27.25 & 1.14 \\
\hline $\mathrm{K}-12$ & 0.0132 & 0.220 & 27.5 & 0.72 \\
\hline-14 & 0.0365 & 0.300 & 27 & 1.46 \\
\hline-15 & 0.0266 & 0.300 & 27 & 1.06 \\
\hline-16 & 0.029 & 0.300 & 27 & 1.16 \\
\hline-17 & 0.0223 & 0.280 & 26.75 & 0.95 \\
\hline-27 & 0.048 & 1.0 & 26.75 & 0.58 \\
\hline $\mathrm{M}-17$ & 0.116 & 1.0 & 26.75 & 1.40 \\
\hline$P-7$ & 0.423 & 7.5 & 24.75 & 0.72 \\
\hline-8 & 0.308 & 7.5 & 24.75 & 0.53 \\
\hline-9 & 0.280 & 5.0 & 27 & 0.68 \\
\hline-10 & 0.483 & 5.0 & 27 & 1.16 \\
\hline-11 & 0.310 & 4.0 & .27 .25 & 0.94 \\
\hline-13 & 0.447 & 4.0 & 27.25 & 1.34 \\
\hline Q-5 & 0.136 & 5.0 & 24.5 & 0.35 \\
\hline \multicolumn{5}{|c|}{$\begin{array}{l}1.53 \pm 0.6 \\
\left(\mathrm{~kg} / \mathrm{m}^{2}\right) /(\mathrm{R} / \mathrm{hr}) \\
\text { average }\end{array}$} \\
\hline
\end{tabular}

${ }^{\mathrm{a}}$ Dose rate at $\mathrm{H}+24$ hours; field readings decayed to $\mathrm{H}+24$ hour $\mathrm{s}$ by $\mathrm{t}^{-1.2}$ decay; tray area $=7.54 \times 10^{-2} \mathrm{~m}^{2}$. 
95 percent of the measured dose from this activity. Prediction of $\mathrm{Na}^{24}$ by present techniques gives an expected yield of $\mathrm{Na}^{24}$ which is 5-6 times greater than the measured yield. This would indicate that the neutron shielding was more effective than calculated. The predictions of activity induced in the soil were made in a very conservative manner by assuming only the minimum thickness of shielding in the device and a moisture content of 3.2 percent. The actual effective thickness of shielding was much greater and the actual moisture content was probably near 10-20 percent.

3.4.2 Fallout Distribution: A plot of infinite dose from $\mathrm{H}+1$ hour versus distance along the hot line is shown in Figure 3.1 along with the predicted curve. A third curve was added to show a comparison with present prediction techniques using actual shot time winds, measured cloud dimensions, and actual fission-product and induced activities calculated to be in the pattern. Close inspection of the curves clearly points up the need for having the very best possible information for predictive purposes.

The original prediction is lower than the measured dose, at ranges beyond about $16 \mathrm{~km}$ for several reasons. First the predicted cloud height was only $2 / 3$ of the observed cloud height, thus tending to bring the activity down sooner. However, the most important reason for the difference in the curves is the fact that the total amount of activity which was predicted to be in the fallout pattern was lower than measured by a factor of about five.

By comparison, the ex post facto calculation stays within a factor of two of the measured pattern from about two base surge radii to a distance of about $100 \mathrm{~km}$. Beyond about $100 \mathrm{~km}$ the ex post facto calculation shows doses increasingly greater than. measured. The prediction system used does not account for wind changes with time and distance and it is highly improbable that the shot time winds were unchanged in $250 \mathrm{~km}$ and 8 to 12 hours. 
Therefore the difference in the curves beyond a few kilometers; can in some degree be accounted for by wind changes.

Another factor which may contribute to the difference between both the original prediction and the ex post facto calculation and the measured curve, is the unknown division of radioactivity between the base-surge cloud and the main cloud. The original prediction and the ex post facto calculation were made assuming 80 percent of the vented activity is injected into the main cloud and 20 percent is injected into the base surge cloud. The effect on the prediction of changing this division is illustrated in Figure 3.9 which shows dose rate at $\mathrm{H}+24$ hours as a function of distance along the hot line. Figure 3.9 also shows the measured dose rate versus distance curve and two curves constructed from ex post facto calculations which represent two radioactivity divisions:

a. 80 percent main cloud, 20 percent base surge;

b. 60 percent main cloud, 40 percent base surge. The 60/40 curve appears to give a better over-all fit to the measured curve than does the $80 / 20$ curve and suggests that the $80 / 20$ division which is used in many prediction techniques may not always be valid. It should be noted that the distinct change in shape of the measured curve at about $100 \mathrm{~km}$ range indicates some change in conditions which is not reflected in the ex post facto calculation curve. A number of factors may contribute to this change but the most likely causes are relatively abrupt changes in winds and terrain.

\subsection{CONCLUSIONS -}

The Sedan detonation gave a dust cloud which was 50 percent larger than predicted and deposited nearly five times more radioactivity than predicted. This indicates that the relationship between depth of burst, yield, and radioactivity escape used to extrapolate to the Sedan event must be re-examined. In addition; 


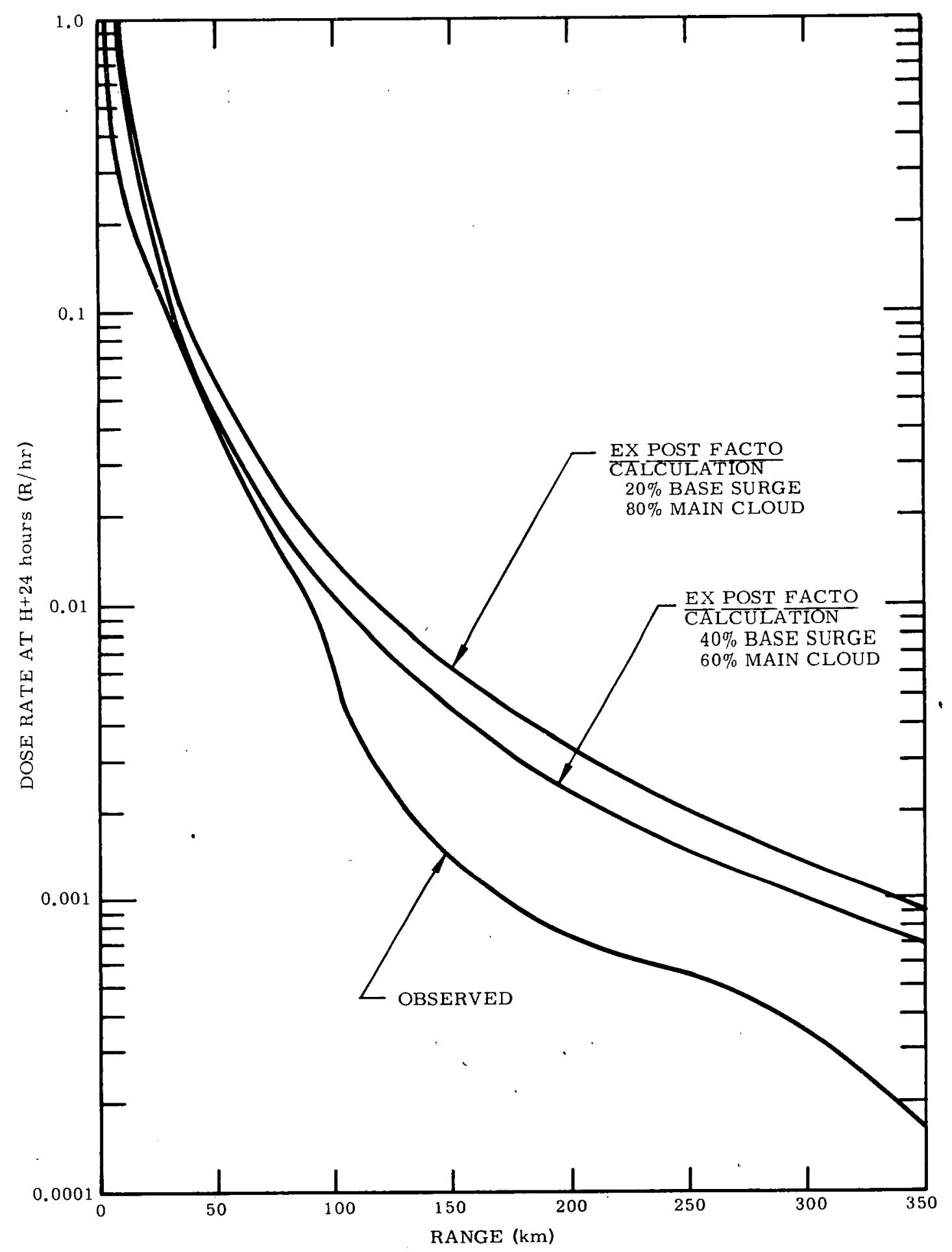

Figure 3.9 Comparison of observed dose rates on the hotline to ex post facto calculations in which activity partition between base surge cloud and main cloud is varied. 
it is also clear that soil moisture content and shot medium chemistry are important in regard to activity escape and cloud height.

Comparison of Sedan results to the present fallout prediction techniques using actual input data shows that the prediction techniques give reasonably accurate results if the basic input data are correct and suggests that the oft-maligned fallout prediction systems may not be as much at fault for poor fallout predictions as are the methods of predicting the input data. 


\section{REFERENCES}

1. G. W. Johnson, Cdr., USN, "Monitor Survey of Ground Contamination (Radsafe), Operation Jangle, "WT-381, Armed Forces Special Weapons Project, Washington, D. C. , May 22, 1952.

2. M. D. Nordyke, "On Cratering, A Brief History, Analysis and Theory of Cratering, "UCRL-6578, Lawrence Radiation Laboratory, Livermore, California, August 22, 1961.

3. M. D. Nordyke and W. R. Wray, "Cratering and Radioactivity Results from a Nuclear Cratering Detonation in Basalt, " UCRL-6999 Rev. II, Lawrence Radiation Laboratory, Livermore, California, October 10, 1963.

4. M. J. Schumchyk and E. H. Bouton, "Fallout Studies, Project 2.5.1, Operation Teapot," WT-1119, U.S. Army Chemical Corps, Chemical Warfare Laboratories, Army Chemical Center, Maryland, July 23, 1958.

5. A. V. Shelton, and others, "The Neptune Event - A Nuclear Explosive Cratering Experiment, "UCRL-5766, Lawrence Radiation Laboratory, Livermore, California, April 19, 1960.

6. A. V. Shelton, Unpublished notes.

7. J. B. Knox, Lawrence Radiation Laboratory, Livermore, California, private communication.

8. W. R. Wray, U. S. Army Corps of Engineers Nuclear Cratering Group; Livermore, California, private communication.

9. J. A. Miskel, "Radiochemical Analysis, Project Sedan," PNE-231F, Lawrence Radiation Laboratory, Livermore, California, to be published.

10. W. B. Lane, "Some Radiochemical and Physical Measurements of Debris from an Underground Nuclear Detonation, Project Sedan, "PNE-229F, U.S. Naval Radiological Defense Laboratory, San Francisco, California, June 1963; K. H. 
Larson, and others,

"Characteristics of Fallout from

a Deeply Buried Nuclear Detonation from about 3 to 150.Miles from Ground Zero, Project Sedan, "PNE-225P, UCLA School of Medicine, Los Angeles, California, August 1962.

11. "Project Sedan - Weather and Surface Radiation Prediction Activities, " PNE-201F, USWB Research Station, Los Vegas, Nevada, November 1962.

12. R. F. Rohrer, Lawrence Radiation Laboratory, Livermore, California, private communication.

13. "Project Sedan - Final Off-Site Report," PNE-200F, U.S. Public Health Service, Off-Site Radiological Safety Organization, Las Vegas, Nevada, December 12, 1962.

14. "Project Sedan - On-Site Radiological Safety Report, " PNE203F, Reynolds Electrical and Engineering Co., Inc., Mercury, Nevada, October 23, 1962.

15. F. L. Vuillemot, Major, USMC, "Naval Aerial Photographic Analysis, Project Sedan, "PNE-230F, U.S. Naval Radiological Defense Laboratory, San Francisco, California, January 1963.

16. G. H. Higgins, Lawrence Radiation Laboratory, Livermore, California, private communication.

17. J. A. Miskel, Lawrence Radiation Laboratory, Livermore, California, private communication.

18. M. M. Williamson, "Fallout Calculations and Measurement," Proceedings of the Third Plowshare Symposium, Engineering with Nuclear Explosives, Lawrence Radiation Laboratory, Livermore, California, April 1964.

19. G. H. Higgins, "Calculation of Radiation Fields from Fallout," UCID-4539, Lawrence Radiation Laboratory, Livermore, California, January 25, 1963. 


\section{CHAPTER 4}

\section{AIR BLAST}

\subsection{CLOSE-IN AIR BLAST}

4.1.1 Experimental Results: In an attempt to measure closein air blast overpressures on Sedan for comparison with earlier data and for extrapolation of our knowledge of air blast to yields such as Sedan, a close-in air-blast measurement program was included on Sedan. For complete results see Reference 1. For this program, nine self-recording air-blast gages were placed along one radial line at distances from ground zero ranging from 305 to 4700 meters. Unfortunately, at most of the stations the air-blast signals greatly exceeded the set range of the gages. In addition, the four nearest stations were not recovered because of the depth of the throwout. As a result of the above, data was obtained at only four ranges. Peak pressures only were obtained at two, one trace required extrapolation to obtain peak value and one performed satisfactorily. Figure 4.1 shows data points for peak overpressures plotted as a function of scaled ground range from ground zero. Previous work ${ }^{1}$ has shown that air-blast effects from cratering detonations is a strong function of the cube-root-scaled depth of burst. Therefore, for comparison with Sedan, a number of curves, derived from similar air-blast studies on chemical and nuclear explosive detonations at approximately the same cuberoot-scaled depth of burial, are shown in Figure 4.1. Details of the se comparable events are given in Table 4.1 .

4.1.2 Previous Experience: Previous experience with chemical explosive cratering detonations had shown that the close-in air-blast characteristically consisted of two pulses or peaks. The first has been attributed to the first motion of the surface of the ground over the detonation point during the spalling phase of the cratering process and has been termed the ground-shock-induced 


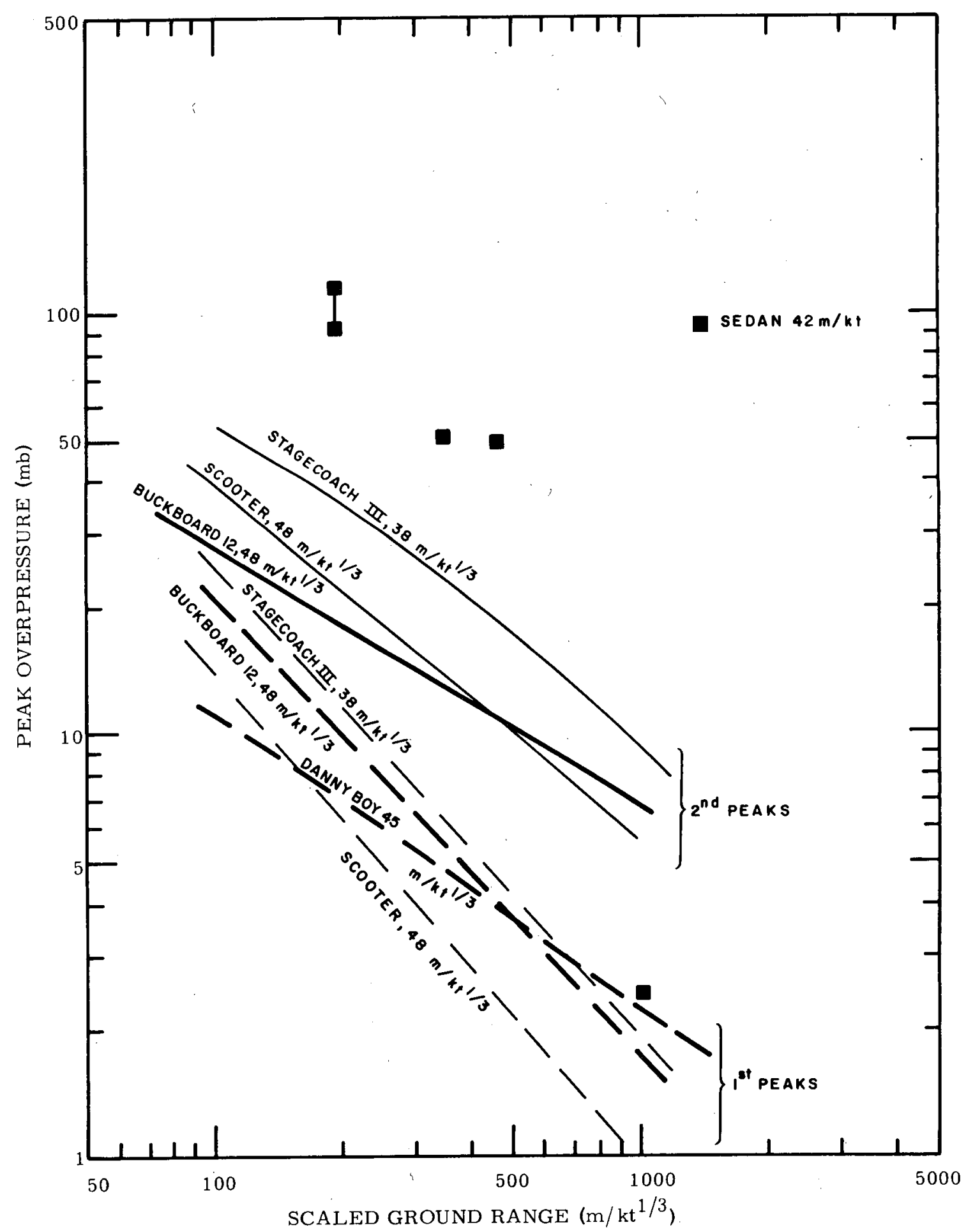

Figure 4.1 Peak air blast vs scaled range. 


\section{TABLE 4.1 DATA FOR FIVE CRATERING EXPLOSIONS}

\begin{tabular}{llccc}
\hline Event & Medium & Yield & $\begin{array}{c}\text { Depth of } \\
\text { Burst }\end{array}$ & $\begin{array}{c}\text { Scaled depth } \\
\text { of Burst }\end{array}$ \\
\hline Stagecoach III & Alluvium & (tons) & $(\mathrm{m})$ & $\left(\mathrm{m} / \mathrm{kt}^{1 / 3}\right)$ \\
Scooter & Alluvium & 500 & 10.4 & 38 \\
Buckboard 12 & Basalt & 20 & 38.1 & 48 \\
Danny Boy & Basalt & 420 & 33.6 & 48 \\
Sedan & Alluvium & 100,000 & 194 & 45 \\
\hline
\end{tabular}

air blast pulse. The second and generally much larger pulse has been attributed to the sudden venting of gases from the explosion cavity at the end of the gas acceleration phase. This behavior has been noted for chemical cratering explosions in alluvium, as well as in basalt. The basalt nuclear cratering explosion, Danny Boy, showed first peaks consistent with the corresponding chemical explosive events (see Figure 4.1). However, the second gas venting pulse was almost totally absent on all records.

\subsubsection{Comparison of Sedan Overpressures with Previous} Experience: In contrast to both the chemical explosive experience and the Danny Boy results, the Sedan signals show only a single pulse, presumably attributed to the large gas vent observed during the Sedan event. As can be seen in Figure 4.1, the signals from three of the stations giving data were a factor of four to five larger than would have been expected for the second peak. Only at the one station at the farthest range was the signal smaller than would be expected for a second peak.

If we define air blast transmissivity factor as the ratio of the air-blast peak overpressure from a buried nuclear explosion to that expected from the same yield nuclear explosion on the surface, we obtain a transmissivity factor for Sedan varying from 
about 0.08 to 0.2 over the range of measurement. These values can be compared with blast suppression factors for the second peaks from the chemical explosive cratering detonations, Scooter and Stagecoach III, which vary from about 0.015 to 0.05 over this same scaled range. Thus, Sedan peak overpressures were transmitted a factor of four to five better than peak overpressure from corresponding lower yield chemical explosive events.

4.1.4 Air Blast Impulse: Pressure versus time traces were obtained at only two stations from which the impulse can be calculated. Figure 4.2 shows these scaled impulses plotted, together with similar data from the other pertinent events. Surprisingly enough, the Sedan points compare very favorably with Scooter, Stagecoach III and Buckboard 12. The Danny Boy curve is much lower, of course, because of the absence of the large gas vent pulse contribution.

\subsubsection{Discussion and Conclusions: The air-blast-impulse} transmission factors (the ratio of the positive phase impulse observed for a subsurface detonation to the positive phase impulse expected for the same yield on the surface) for Sedan are 0.18 and 0.125. These factors are in excellent agreement with impulse transmission factors calculated for Scooter, Stagecoach III and Buckboard 12.

Following a suggestion by Vortman, ${ }^{1}$ a comparison between the air-blast signals from Sedan and Scooter with their respective cavity pressure histories leads to some interesting conclusions. Knox and Terhune, ${ }^{2,3}$ on the basis of code calculations of cavity growth, postshot measurements, and matching of theoretical calculations of cratering mechanics to observed crater dimensions, have derived the cavity pressures and dimensions shown in Table 4.2. Also shown are the observed times of vent of the cavity gases. 


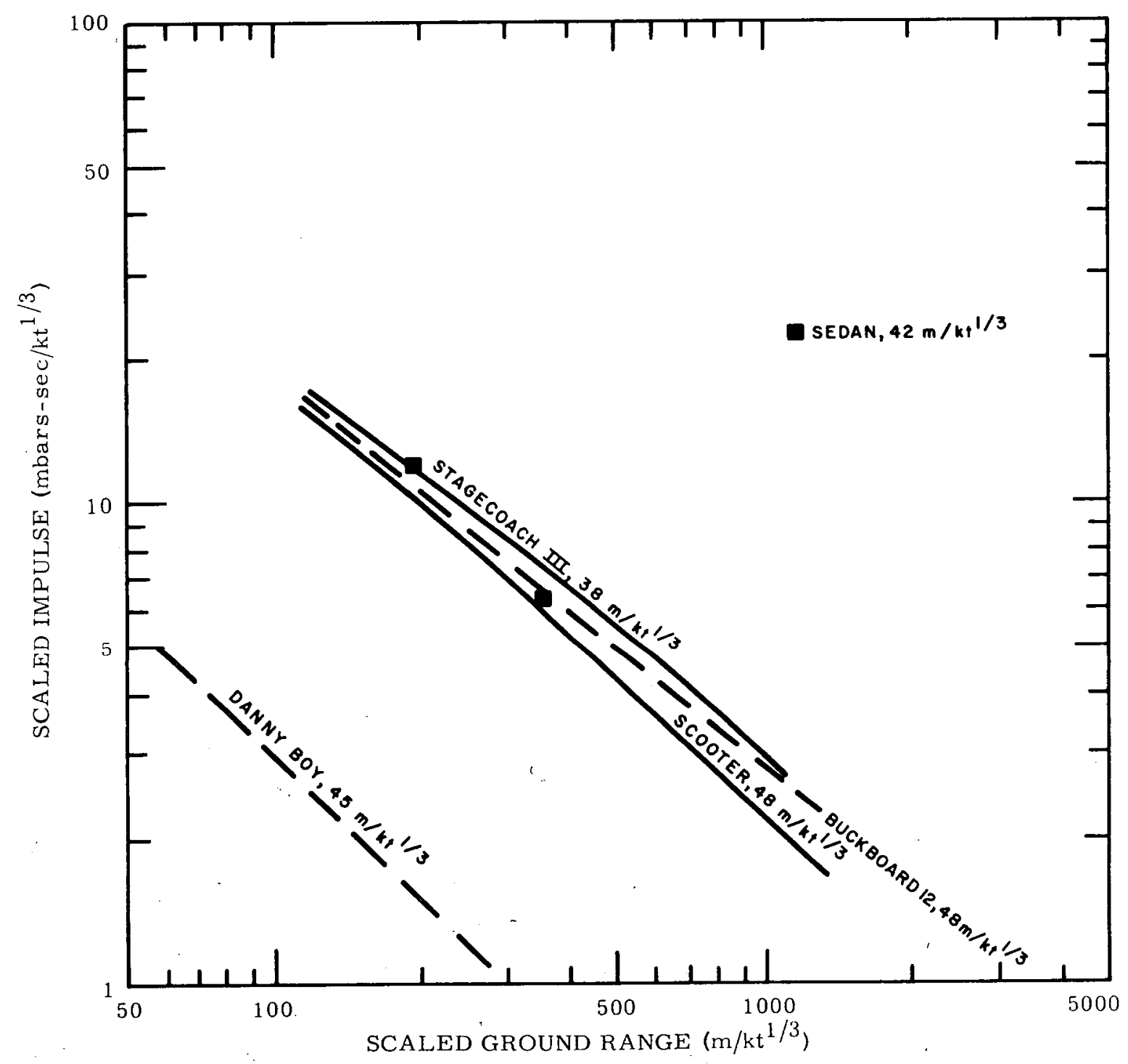

Figure 4.2 Air blast impulse vs scaled range. 
TABLE 4.2 CAVITY DIMENSIONS AND PRESSURES

\begin{tabular}{|c|c|c|c|}
\hline Time & & Sedan & Scooter \\
\hline $\begin{array}{l}\text { When Rarefraction } \\
\text { Returns to Cavity }\end{array}$ & $\begin{array}{l}\text { Cavity Pressure } \\
\text { Cavity Radius } \\
\text { Cavity Volume }\end{array}$ & $\begin{array}{c}600 \text { bars } \\
61 \mathrm{~m} \\
9.5 \times 10^{5} \mathrm{~m}^{3}\end{array}$ & $\begin{array}{c}108 \text { bars } \\
13.7 \mathrm{~m} \\
1.1 \times 10^{4} \mathrm{~m}^{3}\end{array}$ \\
\hline $\begin{array}{l}\text { When Gas Accel- } \\
\text { eration Phase } \\
\text { Begins }\end{array}$ & $\begin{array}{l}\text { Cavity Pressure } \\
\text { Cavity Volume }\end{array}$ & $\begin{array}{c}180 \mathrm{bars} \\
2.8 \times 10^{6} \mathrm{~m}^{3}\end{array}$ & $\begin{array}{r}33 \text { bars } \\
3.5 \times 10^{4} \mathrm{~m}^{3}\end{array}$ \\
\hline $\begin{array}{l}\text { When Venting } \\
\text { Occurs }\end{array}$ & $\begin{array}{l}\text { Cavity Pressure } \\
\text { Cavity Volume }\end{array}$ & $\begin{array}{c}54 \text { bars } \\
8.2 \times 10^{6} \mathrm{~m}^{3}\end{array}$ & $\frac{6.2 \mathrm{bars}}{1.8 \times 10^{5} \mathrm{~m}^{3}}$ \\
\hline & Observed Time & $\sim 3 \mathrm{sec}$ & $-1.1 \mathrm{sec}$ \\
\hline $\begin{array}{l}\text { Effective Gamma } \\
\text { During Gas } \\
\text { Acceleration Phase }\end{array}$ & & 1.12 & 1.03 \\
\hline
\end{tabular}

As can be noted from the cavity pressures listed in Table 4.2

Sedan is initially a factor of six greater than Scooter and at the time of vent is a factor of nine greater. This increase presumably results because Sedan vented at a much earlier scaled time than Scooter.

From the yield ratio of 200 one would expect a vent time for Sedan about six times later than Scooter if the explosion processes scaled anything like $\mathrm{W}^{1 / 3}$, but in reality the ratio of vent time is only about three. The above ratios for cavity pressures between Sedan and Scooter of six to nine are in fairly good agreement with the observed ratio between air-blast peak overpressures. The energy in the gas phase, calculated on the basis of $\mathrm{PV} /(1-\gamma)$, changes only slightly during the gas acceleration phase and so the energy available as a source for the air-blast impulse is roughly independent of vent time. As calculated from the values in Table 4.2, the energy in the gas phase for Sedan is a

- factor of about 100 greater than for Scooter. Because of the strong role played by $\gamma$ in these calculations, they can, at best, be only approximations, but they are consistent with the fact that the impulse measured on Sedan scaled very well to Scooter, using a yield ratio of 200 . 
The above leads to the conclusions, at least qualitatively, that the high peak air-blast overpressures from Sedan can be related to the much higher cavity pressure at the time of vent in the case of Sedan than for Scooter. These high pressures are probably due to (1) Sedan' s venting at a much earlier scaled time than Scooter, and/or (2) the thermodynamics of the nuclear explosion and cavity expansion, which lead to approximately equivalent energy contents, but much higher cavity pressures and smaller cavity radii for nuclear than for chemical explosions at comparable times during the cratering process. In addition, the above leads to the conclusion that the air-blast impulse from a nuclear explosive cratering detonation in alluvium is about equal to the impulse from a corresponding chemical explosion.

Earlier work on damage criteria has related it primarily to peak overpressure, but it is undoubtedly also related to the airblast impulse. The marked difference between the peak overpressure and the impulse for Sedan as compared to Scooter makes it clear that damage criteria must be related not only to peak overpressure but also impulse.

Referring to Table 4.2, it should also be noted that the Sedan cavity radius at the time the rarefraction returns to the cavity is 6 percent smaller than the scaled Scooter cavity at the same scaled time, using $\mathrm{W}^{1 / 3.4}$ scaling. ${ }^{*}$ At the time of vent, the ratio of cavity volumes is about 45 , much smaller than would be expected on the basis of the two yields involved. This indicates the Sedan radius at this time was 25 percent smaller than the scaled Scooter cavity radius. Thus, it is not too surprising that the crater radius

Normally one would scale cavity radius by $\mathrm{W}^{1 / 3}$ but since we are attempting to relate scaled cavity return to scaled cavity dimensions, we have used $\mathrm{w}^{1 / 3.4}$ scaling. 
of Sedan is 18 percent smaller than: predicted on the basis of $\mathrm{w}^{1 / 3.4}$ scaling. The smaller area of vent and the higher pressures present at vent times should lead to much higher vent velocities in the central cone which would eject debris at greater velocities. This would result in more lateral displacement of the ejecta such that a larger fraction of the central material is ejected from the crater. This phenomena could easily lead to the much higher efficiency of nuclear explosions for crater depth than crater radius as has been observed above in Chapter 2 .

\subsection{LONG RANGE AIR BLAST}

Air-blast signals from large-yield explosions may be transmitted by atmospheric refraction to very great ranges with sufficient intensities to produce minor damage such as broken windows, cracked plaster and damaged doors. ${ }^{4}$ To better understand this phenomena and to provide quantitative data on the effect of large yields, a microbarograph program was conducted on the Sedan event. For a complete summary of the results, see Reference 5.

4.2.1 Experimental Plan: Microbarograph records were obtained from eight stations located at ranges of 160 to $370 \mathrm{~km}$. One of the principal factors in long-range transmission of air-blast signals has been determined to be ozonospheric winds at the $100-$ 200,000-foot altitudes. During the summer months these winds characteristically blow from east to west in the northern temperature latitude. Thus the stations for Sedan were located in a $90^{\circ}$ sector centered on a $270^{\circ}$ azimuth from Sedan. Good records were obtained at most stations for both Sedan and the preshot H. E. calibration shot.

4.2.2 Long Range Air-Blast Transmission Factors: Comparison of the signals from Sedan and the 1.2-ton H. E. surface detonation allows calculations to be made of a blast transmission factor 
for long-range air blast. There is considerable scatter in the calculated blast transmission factors attributable to variation in azimuth of the stations, degrees of focussing, and random errors introduced by atmospheric turbulence. Air-blast transmission factors for Sedan varied from 0.05 to 0.35 with a logarithmic average of the most representative values of $0.20 \pm 0.08$. Comparison of these transmission factors with data from similar detonations is given in Table 4.3 .

TABLE 4.3 LONG-RANGE AIR-BLAST TRANSMISSION FACTORS

\begin{tabular}{lcccc}
\hline Event & $\begin{array}{c}\text { Scaled Depth } \\
\text { of Burst }\end{array}$ & \multicolumn{3}{c}{ Transmission Factors } \\
& $\left(\mathrm{m} / \mathrm{kt}^{1 / 3}\right)$ & & & \\
Stagecoach III & 38 & 0.34 & 0.12 & $0.19 \pm 0.05$ \\
Scooter & 48 & 0.37 & 0.11 & $0.19 \pm 0.08$ \\
Buckboard 12 & 48 & 0.31 & 0.13 & $0.21 \pm 0.07$ \\
Danny Boy & 45 & 0.16 & 0.12 & $0.14 \pm 0.01$ \\
Sedan & 42 & 0.35 & 0.05 & $0.20 \pm 0.08$
\end{tabular}

4.2.3 Comparison of Sedan Results with Previous Experience: It is clear from Table 4.4 that the long range air-blast signals from Sedan, as measured by air-blast transmission factors, is completely consistent with all previous experience at this scaled depth of burial, with the exception of the Danny Boy event. The fact that the long-range air-blast peak overpressure transmission factors and close-in air-blast impulse and impulse transmission factors for Sedan are in full agreement with previous chemical explosive cratering experience would appear to confirm that closein air-blast impulse is the most important factor in determining long-range peak overpressures. The order-of-magnitude lower impulse from Danny Boy did not, however, lead to an order-ofmagnitude lower long-range air-blast transmission factor but rather only to reduction by a factor of about 1.5 . 
TABLE 4.4 PREDICTED PEAK-TO-PEAK OVERPRESSURES AT FIRST CAUSTIC RANGE ${ }^{2}$ BASED ON SEDAN UPPER LIMITS

\begin{tabular}{lc}
\hline Yield & Peak-to-Peak Overpressure \\
\hline & $(\mathrm{mb})$ \\
$100 \mathrm{kt}$ (Sedan) & 1 \\
$500 \mathrm{kt}$ & 1.9 \\
$1 \mathrm{Mt}$ & 2.5 \\
$5 \mathrm{Mt}$ & 4.8 \\
$10 \mathrm{Mt}$ & 6.3 \\
$50 \mathrm{Mt}$ & 12.0 \\
\hline
\end{tabular}

\footnotetext{
${ }^{\mathrm{a}}$ Downwind for ozonospheric winds only. Upwind and crosswind values would be an order of magnitude or more lower than these values.
}

4.2.4 Extrapolation of Sedan Results to Larger Yields: The maximum air-blast signals recorded on Sedan in the region of the first caustic range were in the range from 0.5 to 0.9 mbars. If the largest value resulting from Sedan is taken as 1 mbar and this value is scaled to larger yields by $\mathrm{W}^{0.4}$ scaling, ${ }^{1}$ we have the predicted peak-to-peak overpressures shown in Table 4.4. It is difficult to develop useful criteria for air-blast damage but it has been determined ${ }^{4}$ that breakage of large windows 'occasionally occurs at the 2 -mbar level. Smaller windows will take up to 4 to 10 mbars before breakage occurs. If a figure of 4 mbar is taken as a minimum for window breakage, the Sedan results would indicate that up to 2.5 Mt could be fired with Sedan-like focussing and blast transmission factors before significant damage would be expected downwind. If $\mathrm{W}^{0.4}$ scaling does not hold for larger yields or if blast transmission factors should increase with yields above 100 $\mathrm{kt}$, the values in Table 4.4 would be increased accordingly. In addition, at the larger yields overpressures at the second caustic range $(300-500 \mathrm{~km})$ could be large enough to cause damage. 


\section{REFERENCES}

1. L. J. Vortman, "Close-In Air Blast from a Nuclear Event in NTS Desert Alluvium, Project Sedan Final Report," Sandia Corporation, PNE-211F, October 1964.

2. J. B. Knox and R. W. Terhune, "Calculation of ExplosionProduced Craters - High Explosive Sources, " Lawrence Radiation Laboratory, UCRL-7738, Rev. I, October $196 \dot{4}$.

3. R. W. Terhune, Lawrence Radiation Laboratory (Livermore) Private Communication.

4. J. W. Reed, "Air Blast from Cratering Explosions," Proceedings of the Third Plowshare Symposium, April 1964, Lawrence Radiation Laboratory, TID-7695.

5. J. W. Reed and H. W. Church, "Sedan Long Range Blast Propagation," Sandia Corporation, PNE-202F, June 1963. 


\section{CHAPTER 5 \\ GROUND SHOCK}

\subsection{EXPERIMENTAL PLAN}

To document earth motions generated by the Sedan explosion, 11 strong-motion seismographs on-site were operated by USC and GS at ranges from 1.1 to $27 \mathrm{~km}$. In addition, 6 mobile seismographs were operated on a radial line extending northeast of Sedan at approximately $50-\mathrm{km}$ intervals beginning at $150 \mathrm{~km}$. For a more complete discussion of the results of the strong-motion program and the data collected by the long range stations, see Reference 1.

\subsection{EXPERIMENTAL RESULTS}

Peak acceleration and displacements recorded by the strongmotion stations on Sedan are shown in Figures 5.1 and 5.2, where all three components of motion are plotted. In addition, the data are segregated with respect to whether the stations were on the east or west side of the Yucca Fault. This fault is a major northsouth geologic feature of Yucca Valley which passes about $1.4 \mathrm{~km}$ west of the Sedan ground zero. Also shown in Figures 5.1 and 5.2 are the prediction functions

$$
a=0.0041 w^{0.54} R^{-1.4}
$$

and

$$
d=0.0027 \mathrm{w}^{0.8} \mathrm{R}^{-1.2},
$$

where

$$
\begin{aligned}
\mathrm{a} & =\text { peak earth particle acceleration, } \mathrm{g} ; \\
\mathrm{d} & =\text { peak earth particle displacement, } \mathrm{cm} ; \\
\mathrm{W} & =\text { equivalent yield, tons; } \\
\mathrm{R} & =\text { range from } \mathrm{GZ} \text { to station, } \mathrm{km} .
\end{aligned}
$$




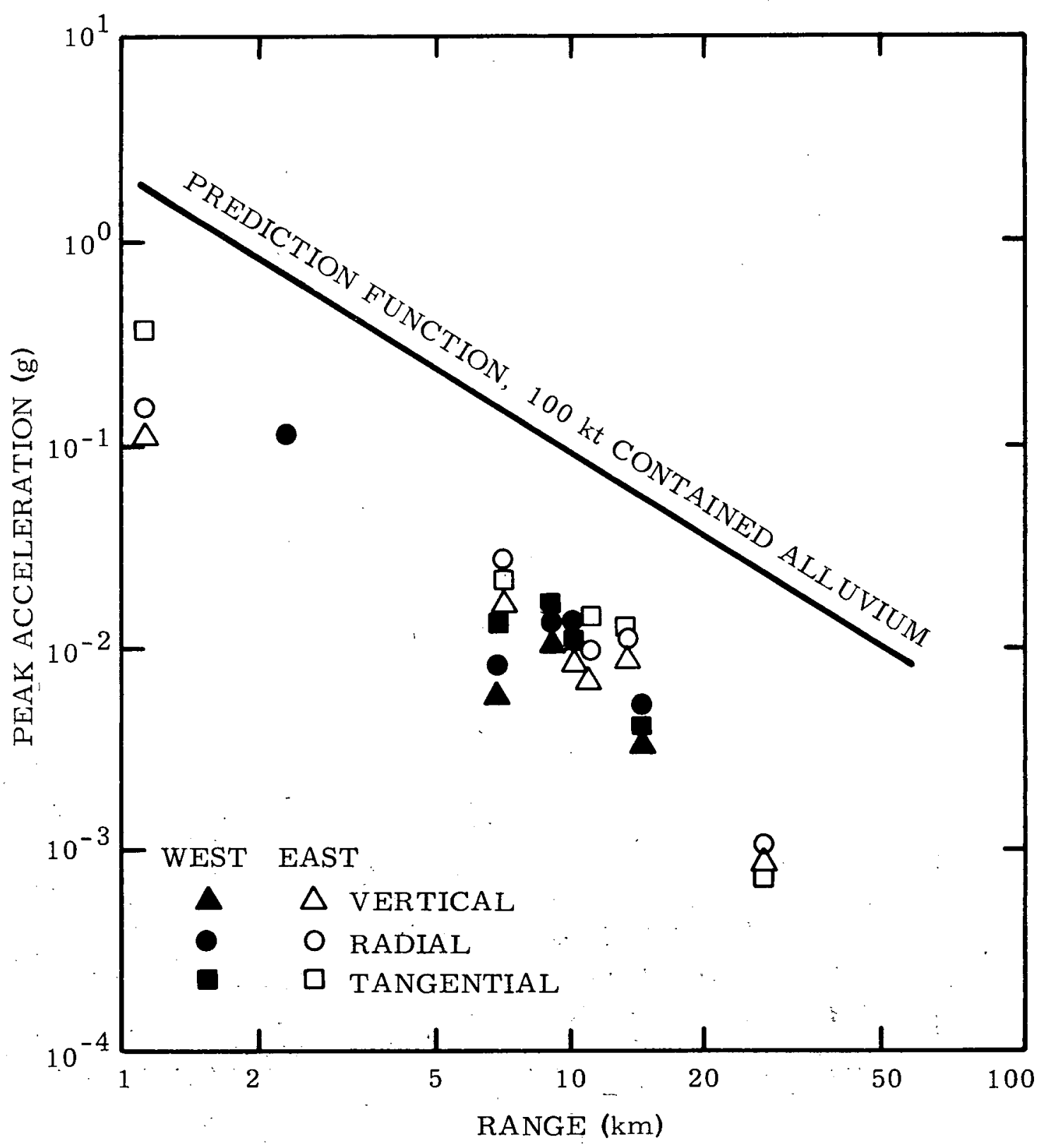

Figure 5.1 Peak accelerations for Sedan. 


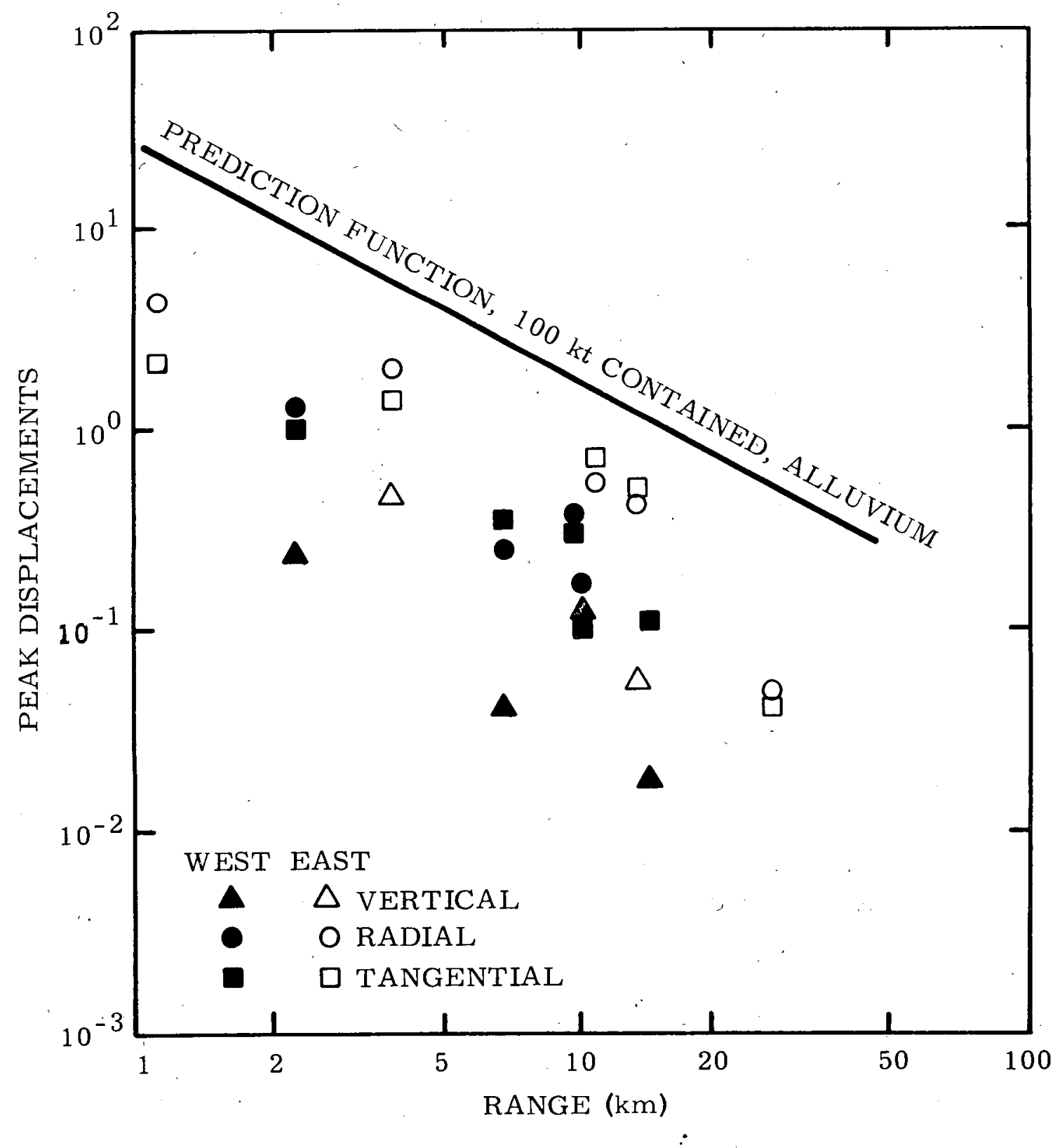

Figure 5.2 Peak displacements for Sedan. 
These functions were derived from strong motion data measurements on a wide variety of nuclear detonations in alluvium at a number of yields, all of which were below $100 \mathrm{kt}^{2}$ These functions are not best-value fits to the data but rather represent an upper limit to the observed data such that 90 to 95 percent of the measured data points fall below the curve.

\subsection{DISCUSSION AND CONCLUSIONS}

Acceleration data from stations located on the west or opposite side of the fault from Sedan are somewhat below the data at the same range on the east side but for displacement there is a very clear and systematic differentiation between the data from the two sides of the fault. Displacements on the opposite side of the fault were consistently a factor of two to three below displacements on the same side. Comparison of the data with the prediction functions shows that Sedan produced accelerations a factor of about five less than the same yield would have as a completely contained shot. Displacement indicates a reduction of about a factor of two to five depending generally on which side of the fault the stations were located.

Direct comparison between Sedan and surface motion data from a completely contained nuclear explosion of similar yield in the tuff underlying Yucca Flat shows Sedan to have somewhat lower accelerations and approximately equal displacements over the range from 3 to $20 \mathrm{~km}$. When the effect of the difference in shot environment is compensated for, the two shots would compare very favorably, considering the large inherent scatter in surface motion data.

Thus, it cannot be concluded from the Sedan data that cratering explosions in alluvium produce significantly less motion than similar contained shots. The prediction functions given above would appear to overestimate surface motion for a shot such as 
Sedan by perhaps as much as a factor of two to five. Use of these functions for extrapolation to large yields must be carefully examined since they were developed on the basis of data from shots smaller than Sedan and the overestimation of Sedan could well be a result of too large an exponent for $\mathrm{W}$. 


\section{REFERENCES}

1. W. V. Mickey, "Seismic Effects from a High Yield Nuclear Cratering Experiment in Desert Alluvium, "U.S. Coast and Geodetic Survey, PNE-213F, April 1963.

2. W. V. Mickey, "Seismic Wave Propagation," Proceedings of the Third Plowshare Symposium, April 1964, TID-7695. 


\section{CHAPTER 6 \\ MASS DISTRIBUTION}

\subsection{EJECTA AND THROWOUT THICKNESS}

\subsubsection{Experimental Plan and Results: In an effort to}

measure the distribution and character of the throwout, ejecta, and missiles surrounding the Sedan crater, an extensive array of tarps, trays and height rods were placed in the area around the Sedan ground zero. ${ }^{1}$ On the basis of postshot measurements in this array, an ejecta isopach map has been developed (see' Figure 6.1). This shows the ejecta distribution is fairly isotropic, covering an area up to $1.3 \mathrm{~km}$ in radius with dust ranging in thickness from $1 \mathrm{~cm}$ on the edge up to 10 meters in the general area of the crater lip.

Beyond $1.3 \mathrm{~km}$, dust was deposited by the expanding base surge out to the limit of its radial growth. This layer is readily apparent in the aerial photo mosaic of the Sedan area (Figure 3.5). As discussed in Section 3.3.4, the mass per unit area can be fairly well correlated with the external gamma field readings, wherein the data in Table 3.2 give a mass thickness factor of $1.53 \pm 0.67$ $\mathrm{kg} / \mathrm{m}^{2}$ per $\mathrm{R} / \mathrm{hr}$ at $\mathrm{H}+24$ hours. Using a measured postshot density of $1.5 \mathrm{gm} / \mathrm{cm}^{3}\left(1500 \mathrm{~kg} / \mathrm{m}^{3}\right)^{1}$, this mass density factor may be converted to a mass thickness factor of approximately $1 \pm 0.5 \mathrm{~mm}$ per $\mathrm{R} / \mathrm{hr}$. Thus, the $\mathrm{H}+24$ hour fallout patterns shown in Figures 3.3 and 3.4 may be used for dust isopach maps by reading the $R / h r$ contour in terms of millimeters for radial distances beyond 1.3 $\mathrm{km}$ or for thicknesses less than $1 \mathrm{~cm}$.

Figure 6.2 shows the mass of material per unit area as a function of range where data from all azimuths have been averaged. A least-squares fit to the azimuthally averaged data points gives the functions 



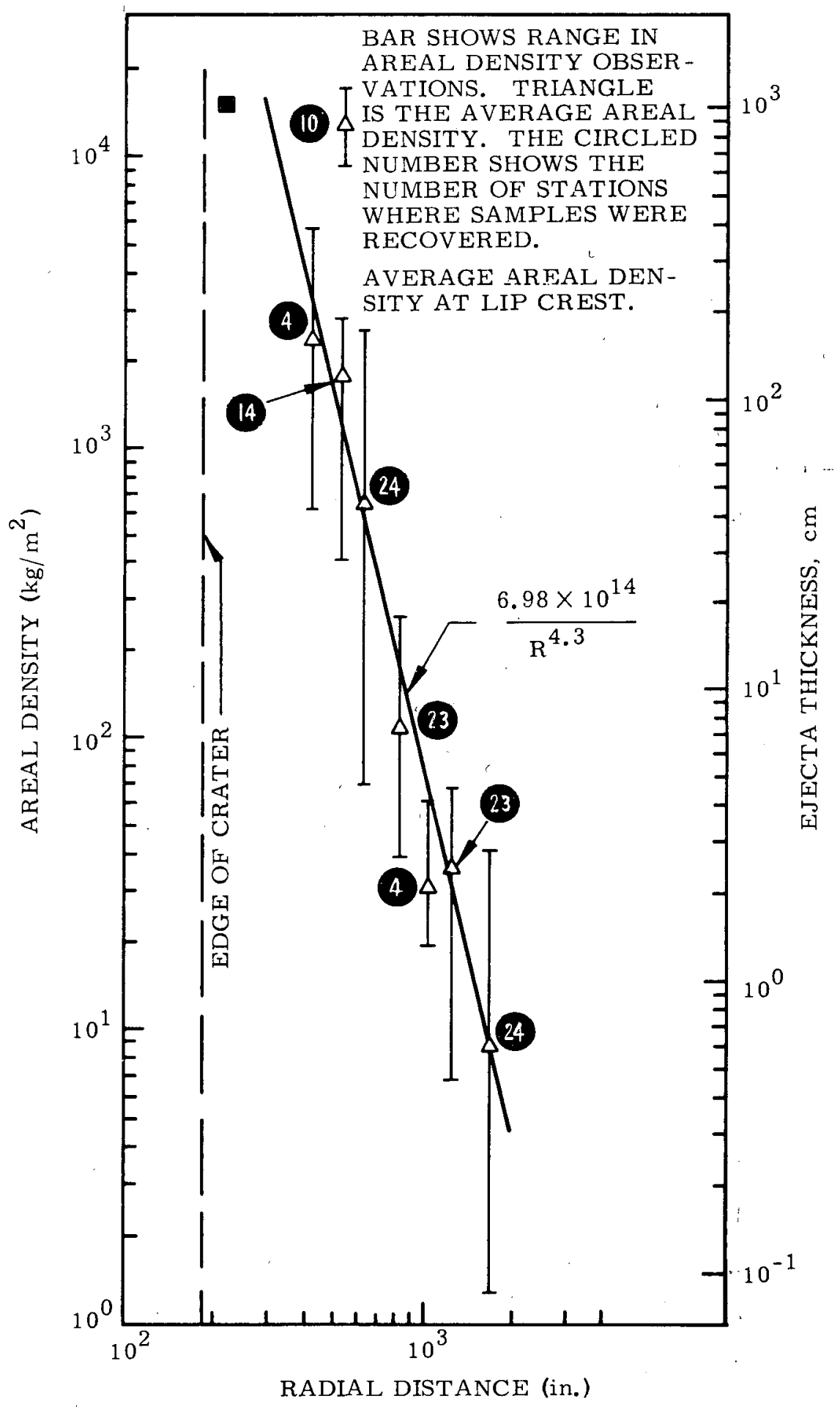

Figure 6.2 Mass of material per unit areas as a function of range. 


$$
\delta=\frac{6.98 \times 10^{14}}{\mathrm{R}^{4.3}}
$$

and

$$
t=\frac{4.65 \times 10^{13}}{R^{4.3}}
$$

where

$$
\begin{aligned}
\delta & =\text { areal density, } \mathrm{kg} / \mathrm{m}^{2} ; \\
\mathrm{t} & =\text { ejecta thickness, } \mathrm{cm} ; \\
\mathrm{R} & =\text { range from ground zero, meters. }
\end{aligned}
$$

Such a fit would appear to give the ejecta thickness within about a factor of three on the average. If three functions are used to fit the data, we have

$$
\begin{array}{ll}
\delta_{1}=\frac{2.26 \times 10^{9}}{\mathrm{R}^{2.2}} & 220 \leq \mathrm{R} \leq 550 \text { meters } \\
\delta_{2}=\frac{3.88 \times 10^{19}}{\mathrm{R}^{6.0}} & 550 \leq \mathrm{R} \leq 840 \text { meters } \\
\delta_{3}=\frac{2.60 \times 10^{12}}{\mathrm{R}^{3.5}} & 840 \leq \mathrm{R} \leq 1710 \text { meters. }
\end{array}
$$

6.1.2 Discussion and Conclusions: Using the above fits and topographic and geologic data for the lip, the mass of ejecta can be calculated. ${ }^{1}$ This integration shows that the ejecta mass represents about 58 percent of the mass corresponding to the apparent crater volume. Of the total ejecta mass, 50 percent was deposited within two crater radii of ground zero; 80 percent was within three crater radii and 97 percent within 10 crater radii. Such an ejecta mass fraction and distribution compares very favorably with previous experience with chemical and nuclear detonations at this scaled depth of burial. 
The above leads to the conclusion that the yield dependence of ejecta should be of the form

$$
\frac{\delta}{\mathrm{W}^{1 / 3.4}}=\mathrm{K}\left(\mathrm{R} / \mathrm{W}^{1 / 3.4}\right)^{-\mathrm{b}}, \mathrm{b}>2 \text {, }
$$

where $\mathrm{W}$ = equivalent yield, kt. Normalizing this equation to Sedan we have an equation for ejecta thickness for alluvium of

$$
\delta / \mathrm{W}^{1 / 3.4}=5.3 \times 10^{11}\left(\frac{\mathrm{R}}{\mathrm{w}^{1 / 3.4}}\right)^{4.3}
$$

or

$$
\delta=\frac{5.3 \times 10^{11} \mathrm{w}^{1.56}}{\mathrm{R}^{4.3}}
$$

This function predicts the averaged ejecta distribution reported for the $0.5 \mathrm{kt}$ Scooter event ${ }^{2}$ within a factor of 1.5 to 2.0 .

\subsection{IMPACT CRATERS}

In addition to the general mass of material ejected from the crater as a more or less homogeneous mass, a large number of agglomerated masses of alluvium received significantly higher velocities than the average and followed ballistic trajectories. When these missiles struck the surface they produced impact craters of various size depending on their mass, velocity, and impact angle. Figure 6.3 is an oblique photo of the Sedan area showing many of these impact craters in the foreground. Many hundred impact, craters were noted at ranges from ground zero as great as 2,140 meters. Numerous impact craters with lip-to-lip diameters of up to 10 meters were located out to radial distances of almost 1300 meters. The largest impact crater noted was located about 300 meters from ground zero and was about 40-60 meters in diameter.

A complete discussion of this program can be found in Reference 1 . 


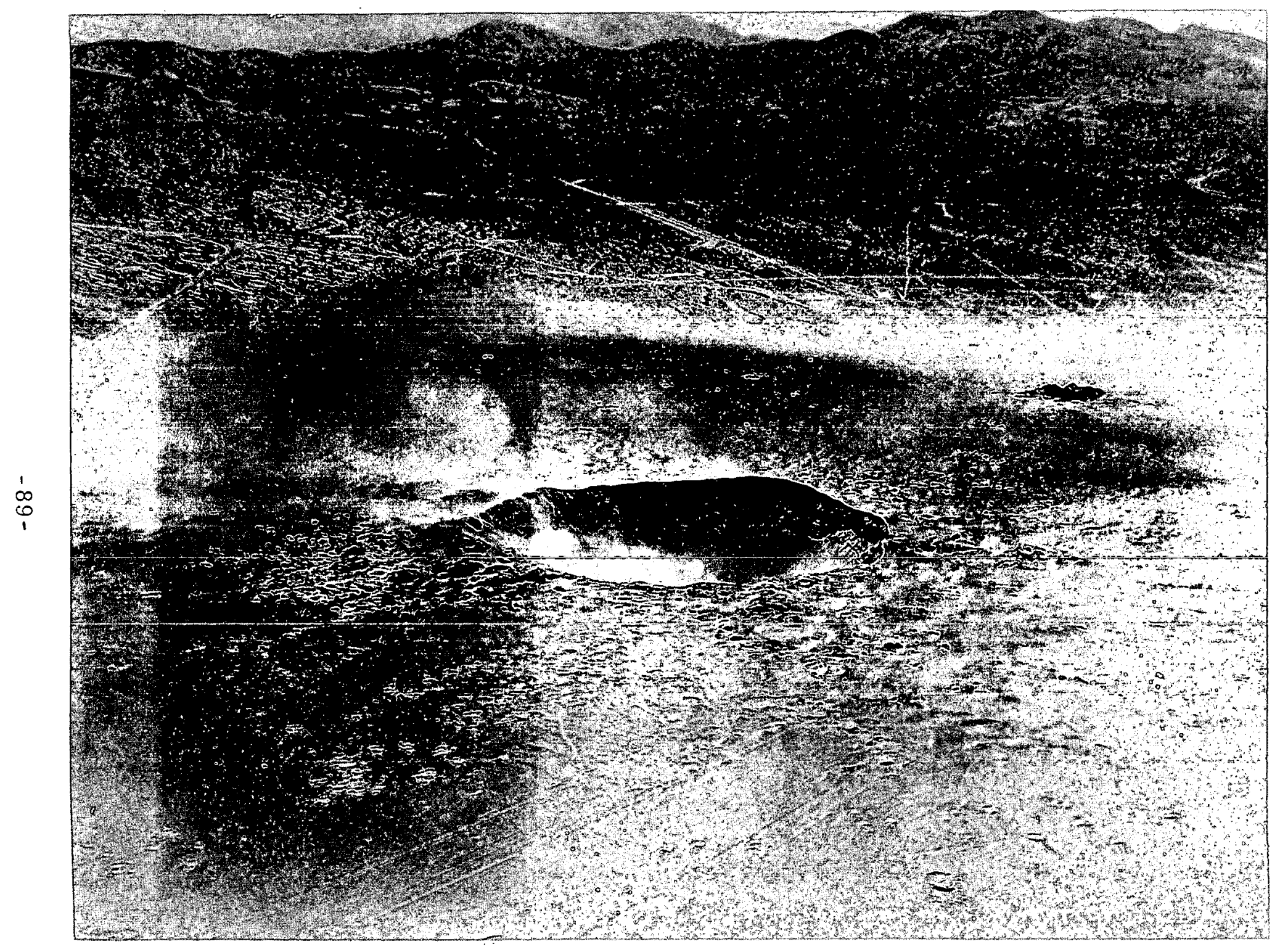

Figure 6.3 Sedan crater; note impact craters in foreground. 


\section{REFERENCES}

1. R. H. Carlson and W. A. Roberts, "Mass Distribution and Throwout Studies, Project Sedan, "The Boeing C'ompany, PNE-217F, August 1963.

2. W. R. Perret and others, "Project Scooter, Final Report," Sandia Corporation SC-4602(RR), October 1963. : 
speed in the alluvium of $640 \mathrm{~m} / \mathrm{sec}$ which is consistent with the range of measured values. This arrival time has been used to establish the time base for the plots in Figure 7.1.

Figure 7.2 shows a plot of vertical velocity as a function of time for the three targets as derived from the smoothed displacement data in Figure 7.1. Target 4 was obscured at about 1.3 seconds by dust but reappeared at about 2.2 seconds and the missing portion of the displacement and velocity data have been sketched in.

A large vent of incandescent gases occurred at 2.8 seconds, generally obscuring any further target motion. The vertical velocity of this vent, over the first 800 feet of its path, was approximately $330 \mathrm{~m} / \mathrm{sec}$. A second vent with about the same velocity occurred at about 3.1 seconds and was very quickly followed by general venting throughout the dome. Figure 1.2 shows the vent at 2.8 seconds as seen from a camera station on the southeast azimuth and Figure 7.3 shows both vents at 3.1 seconds as viewed from a camera station on the southwest azimuth. The asymmetry leading to the high lip on the north-northwest side of the crater is still clearly visible on the left side in Figure 7.3.

\subsection{DISCUSSION}

The velocity versus time plots in Figure 7.2 are, qualitatively, very similar to previous plots for the 0.5 -kiloton Scooter event, ${ }^{1}$ showing an initial spall velocity, followed by a period of free fall, which is followed in turn at about 0.9-1.0 second by a period of sustained gas acceleration lasting generally until venting.

Qualitatively, the initial spall velocities of Sedan compare quite favorably with previous experience in alluvium. On the basis of a number of chemical explosive cratering detonations in desert alluvium with yields ranging from about 0.1 ton to 500 tons (including Scooter), Vortman ${ }^{3}$ has derived the following expression for 


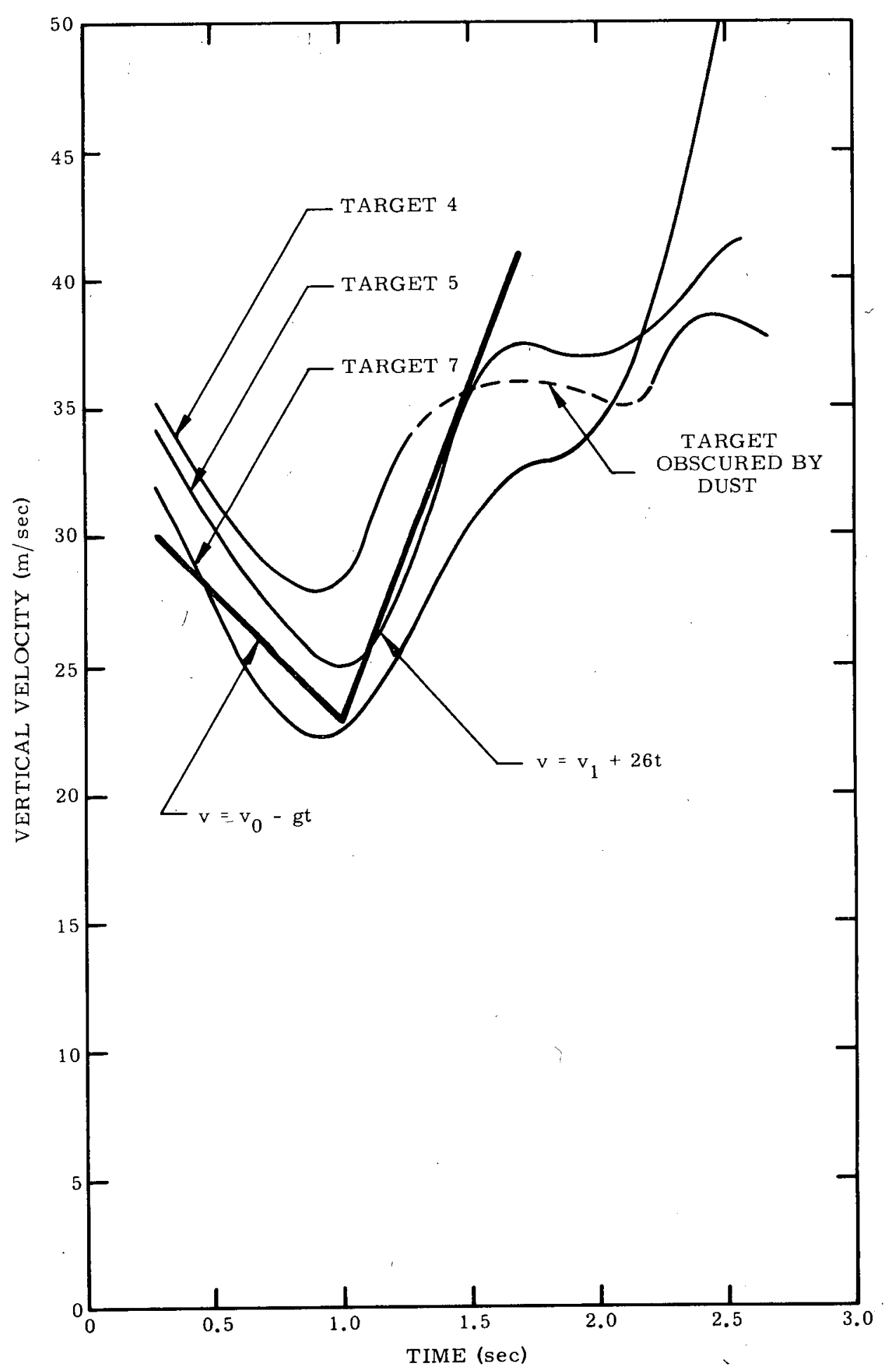

Figure 7.2 Vertical velocity as a function of time. 


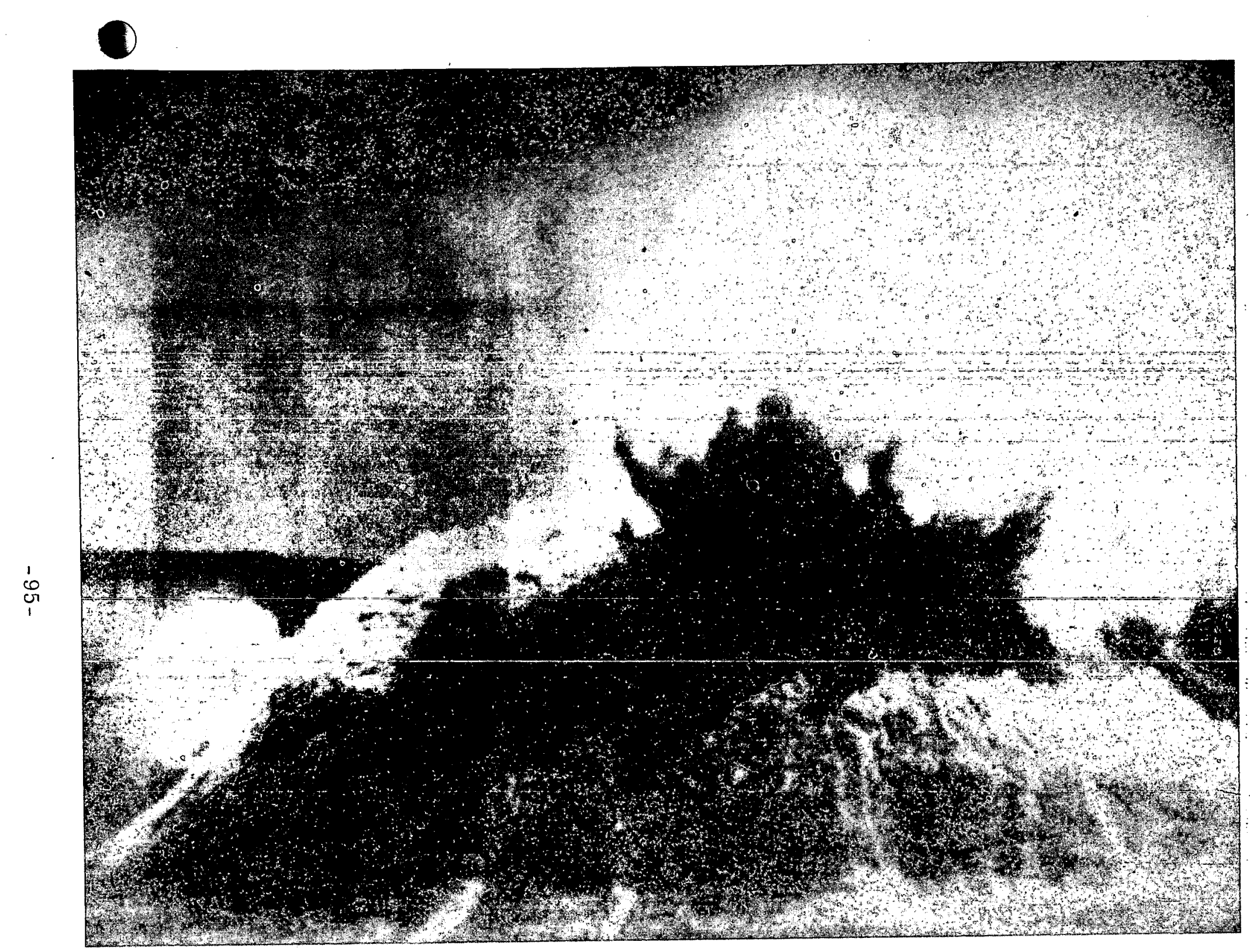

Figure 7.3 Sedan venting at 3.1 seconds. 
surface spall velocity as a function of scaled depth of burial:

$$
\mathrm{v}=1.6 \times 10^{6}\left(\frac{\mathrm{Z}}{\mathrm{w}^{1 / 3}}\right)^{-2.3},
$$

where

$$
\begin{aligned}
\mathrm{v} & =\text { vertical spall velocity, } \mathrm{m} / \mathrm{sec} ; \\
\mathrm{Z} & =\text { depth of burial, meters; } \\
\mathrm{W} & =\text { equivalent yield, kt. }
\end{aligned}
$$

For Sedan, this expression gives a velocity of about $30 \mathrm{~m} / \mathrm{sec}$ for ground zero. Sedan spall velocities, as measured by targets 4 , 5 , and 7 , were $35 \mathrm{~m} / \mathrm{sec}, 34 \mathrm{~m} / \mathrm{sec}$ and $32 \mathrm{~m} / \mathrm{sec}$, respectively. Correction of the predictions for the slant range of targets 4 and 7 is less than 3 percent. Thus, the observed Sedan spall velocities were about 10 percent larger than would have been predicted on the basis of past experience.

The period of negative acceleration has a slope of about -1.5 $g$ and lasts a period of time significantly shorter than would have been expected on the basis of data from Scooter. The gas acceleration phase for Scooter began to be apparent in the surface motion at about $350 \mathrm{msec}$, which would scale, for Sedan, to about 2.0 seconds. This together with the discrepancy in scaled vent times noted in Section 4.1.5, clearly indicates that the gas acceleration phase of the cratering process does not scale in a simple way with the yield but is a complex function of the pressure in the cavity, the size of the cavity, and the mass of the overburden.

Following the simple gas-acceleration model suggested in Reference 1, an estimate of the magnitude of the gas acceleration can be made from the equation

$$
a=\frac{1.15 \times 10^{5} \mathrm{PV}^{2 / 3}}{\mathrm{R}^{3} \rho}
$$


where

$\mathrm{a}=$ gas acceleration, $\mathrm{m} / \mathrm{sec}^{2}$;

$P=$ cavity pressure at the beginning of the gas acceleration phase, bars;

$\mathrm{V}=$ cavity volume at the beginning of the gas acceleration phase, $\mathrm{m}^{3}$;

$R=$ distance from detonation point to the top of the hemispherical dome at the beginning of the gas acceleration phase, m;

$\rho=$ average density of overburden, $\mathrm{kg} / \mathrm{m}^{3}$.

From Figure 7.1 we have

$$
R=Z+h=194+20=214 m \text {. }
$$

Using the data in Table 4.2 and a density of $1600 \mathrm{~kg} / \mathrm{m}^{3}$, we have

$$
\mathrm{a}=26 \mathrm{~m} / \mathrm{sec}^{2} \text {. }
$$

A curve has been constructed on Figure 7.2 using the predicted spall velocity, the observed period of free fall, followed by the above calculated gas acceleration. This curve compares very favorably with the observed velocities until about 1.5-1.6 seconds. Obviously, a more sophisticated version of this model including the adiabatic change in the cavity pressure, the effect of changing overburden with angle from the vertical, and frictional shear losses must be used to explain the late-time behavior. Such a model is being developed ${ }^{2}$ and more complete calculations of the Sedan èvent will be published at a later time. ${ }^{4}$

\subsection{CONCLUSION}

Observed surface motion in the Sedan event agrees very well with previous experience with respect to initial spall velocities and the model of gas acceleration developed at LRL. The length of the period of free fall between the initial spall motion and the beginning 
of the observable gas acceleration phase does not scale from chemical to nuclear in proportion to the cube root of the yield, but will require the development of a more detailed and sophisticated model.

\subsection{ACKNOWLEDGMENT}

The author would like to express a special debt of gratitude to R. W. Terhune and B. Myers who spent many hours reading the Sedan film and processing the data used in this section. 


\section{REFERENCES}

1. M. D. Nordyke, "Nuclear, Craters and Preliminary Theory of the Mechanics of Explosive Crater Formation, "J. Geophysical Research, 66, 10, 3439-59, 1961.

2. J. B. Knox and R. W. Terhune, "Calculation of Explosion Produced Craters - High Explosive Sources, "Lawrence Radiation Laboratory, UCRL-7738 Rev. I, October 1964.

3. W. R. Perret, L. J. Vortman, et al., "Project Scooter, Final Report," Sandia Corporation, SC-4602(RR), October 1963.

4. J. B. Knox and R. W. Terhune, Report to be published. 
TECHNICAL REPORTS SCHEDULED FOR ISSUANCE BY AGENCIES PARTICIPATING IN PROJECT SEDAN

AEC REPORTS

\begin{tabular}{|c|c|c|c|}
\hline AGENCY & PNE NO. & & SUBJECT OR TITLE \\
\hline USPHS & $200 F$ & 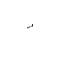 & Off-Site Radiation Safety \\
\hline USW B & $201 F$ & & $\begin{array}{l}\text { Analysis of Weather and Surface Radiation } \\
\text { Data }\end{array}$ \\
\hline $\mathrm{SC}$ & $202 \mathrm{~F}$ & & Long Range Blast Propagation \\
\hline REECO & $203 F$ & & On-Site Rad-Safe \\
\hline AEC/USBM & $204 \mathrm{~F}$ & & $\begin{array}{l}\text { Structural Survey of Private Mining Opera- } \\
\text { tions }\end{array}$ \\
\hline FAA & $205 F$ & & Airspace Closure \\
\hline SC & $211 \mathrm{~F}$ & & $\begin{array}{l}\text { Close-In Air Blast From a Nuclear Event in } \\
\text { NTS Desert Alluvium }\end{array}$ \\
\hline LRL-N & $212 P$ & & Scientific Photo \\
\hline LRL & $214 P$ & & Fallout Studies \\
\hline LRL & $215 \mathrm{~F}$ & & Structure Response \\
\hline LRL & $216 P$ & & Crater Measurements \\
\hline Boeing & $217 P$ & & Ejecta Studies \\
\hline LRL & $218 \mathrm{P}$ & & Radioactive Pellets \\
\hline USGS & $219 \mathrm{~F}$ & & Hydrologic Effects, Distance Coefficients \\
\hline USGS & $221 \mathrm{P}$ & & Infiltration Rates Pre and Post Shot \\
\hline UCLA & $224 P$ & ! & $\begin{array}{l}\text { Influences of a Cratering Device on Close-In } \\
\text { Populations of Lizards }\end{array}$ \\
\hline UCLA & $\begin{array}{l}225 \mathrm{P} \\
\mathrm{Pt} . \mathrm{I} \text { and }\end{array}$ & II & Fallout Characteristics \\
\hline
\end{tabular}


TECHNICAL REPORTS SCHEDULED FOR ISSUANCE

BY AGENCIES PARTICIPATING IN PROJECT SEDAN

\begin{tabular}{|c|c|c|}
\hline AGENCY & PNE NO. & SUBJECT OR TITLE \\
\hline BYU & $226 \mathrm{P}$ & $\begin{array}{l}\text { Close-In Effects of a Subsurface Nuclear } \\
\text { Detonation on Small Mammals and Selected } \\
\text { Invertabrates }\end{array}$ \\
\hline UCLA & $228 \mathrm{P}$ & Ecological Effects \\
\hline LRL & $231 F$ & Rad-Chem Analysis \\
\hline LRL & $232 \mathrm{P}$ & Yield Measurements \\
\hline EGG & $233 P$ & Timing and Firing \\
\hline W ES & $234 \mathrm{P}$ & Stability of Cratered Slopes \\
\hline LRL & $235 \mathrm{~F}$ & Seismic Velocity Studies \\
\hline
\end{tabular}

\section{DOD REPORTS}

AGENCY $\quad$ PNE NO.

USC-GS

NRDL

NRDL

\section{$213 P$}

$229 P$

$230 P$
SUBJECT OR TITLE

"Seismic Effects From a High Yield Nuclear Cratering Experiment in Desert Alluvium"

"Some Radiochemical and Physical Measurements of. Debris from an Underground Nuclear Explosion"

Naval Aerial Photographic Analysis 


\section{ABBREVIATIONS FOR TECHNICAL AGENCIES}

STL Space Technology Laboratories, Inc., Redondo Beach, Calif.

SC

Sandia Corporation, Sandia Base, Albuquerque, New Mexico

USC\&GS U. S. Coast and Geodetic Survey, San Francisco, California

LRL Lawrence Radiation Laboratory, Livermore, California

LR L-N Lawrence Radiation Laboratory, Mercury, Nevada

Boeing The Boeing Company, Aero-Space Division, Seattle 24, Washington

USGS Geological Survey, Denver, Colorado, Menlo Park, Calif., and Vicksburg, Mississippi

WES USA Corps of Engineers, Waterways Experiment Station, Jackson, Mississippi

EGG Edgerton, Germeshausen, and Grier, Inc., Las Vegas, Nevada, Santa Barbara, Calif., and Boston, Massachusetts

BYU Brigham Young University, Provo, Utah

UC LA UCLA School of Medicine, Dept. of Biophysics and Nuclear Medicine, Los Angeles, Calif.

NRDL Naval Radiological Defense Laboratory, Hunters Point, Calif.

USPHS U. S. Public Health Service, Las Vegas, Nevada

USWB U. S. Weather Bureau, Las Vegas, Nevada

USBM U. S. Bureau of Mines, Washington, D. C.

FAA Federal Aviation Agency, Salt Lake City,' Utah

REECO Reynolds Electrical and Engineering Co., Las Vegas; Nevada 
PNE NO. DIST. CAT. PNE NO. DIST.CAT. PNE NO. DIST. CAT.

$\begin{array}{llllll}200 & 26,28 & 214 & 26 & 226 & 42 \\ 201 & 2,26 & 215 & 32 & 228 & 42 \\ 202 & 12 & 216 & 14 & 229 & 26,22 \\ 203 & 28 & 217 & 14 & 230 & 100 \\ 204 & 32 & 218 & 12,14 & 231 & 22 \\ 205 & 2 & 219 & 14 & 232 & 4 \\ 211 & 12 & 221 & 14 & 233 & 2 \\ 212 & 92,100 & 224 & 42 & 234 & 14 \\ 213 & 12,14 & 225 & 26 & 235 & 14\end{array}$

In addition, one copy of reports $201,202,203,211,214,215,216,217$, $218,221,225,229,230,232,234$, and 235 to each of the following:

The Rand Corp.

1700 Main St.,

Santa Monica, California

Attn: Mr. H. Brode

U. of Illinois, Civil Engineering Hall

Urbana, Illinois

Attn: Dr. N. Newmark

Stanford Research Institute Menlo Park, California

Attn: Dr. Vaile

E. H. Plesset Associates 1281 Westwood Blvd. , Los Angeles 24, California

Attn: Mr. M. Peter
Mitre Corp.

Bedford, Massachusetts

General American Transportation Corp. Mechanics Research Div.

7501 N. Natchez Ave.,

Niles 48, Illinois

Attn: Mr. T. Morrison; Dr. Schiffman

Dr. Whitman

Massachusetts Institute of Technology

Cambridge, Massachusetts 